\title{
Matching rules and substitution tilings
}

\author{
Chaim Goodman-Strauss
}

April 25, 1996

1

for Raphael Robinson

\begin{abstract}
A substitution tiling is a certain globally defined hierarchical structure in a geometric space; we show that for any substitution tiling in $E^{n}, n>1$, subject to relatively mild conditions, one can construct local rules that force the desired global structure to emerge. As an immediate corollary, infinite collections of forced aperiodic tilings are constructed.
\end{abstract}
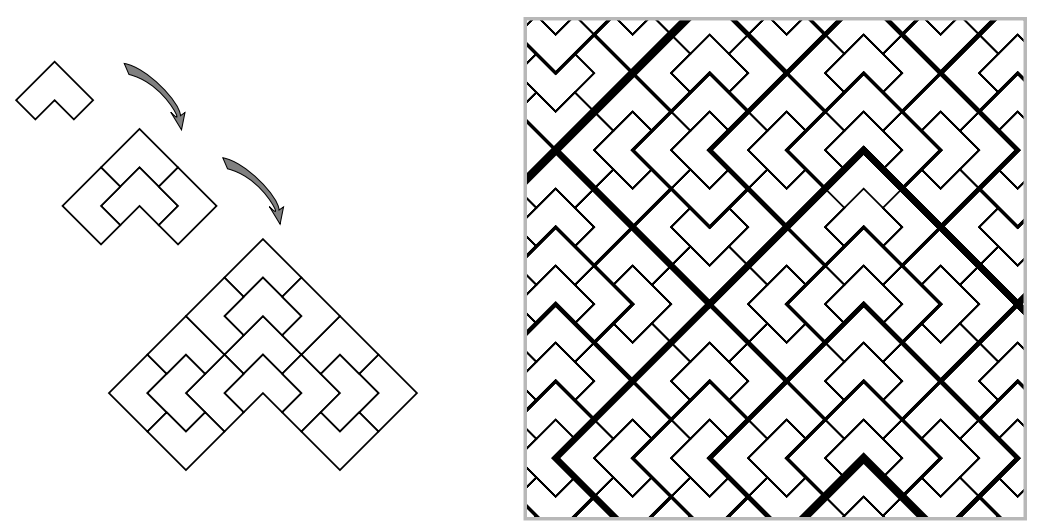

Figure 1: A substitution tiling

On the left in figure $1 \Gamma \mathrm{L}$-shaped tiles are repeatedly "inflated and subdivided". (We define our terms more precisely in Section 1.) As this process is iterated larger and larger regions of the plane are tiled with L-tiles hierarchically

\footnotetext{
${ }^{1}$ Dept. of Mathematics, Univ. Arkansas, Fayetteville, AR 72701. This work was partially supported by the Geometry Center under NSF Grant DMS-8920161.
} 

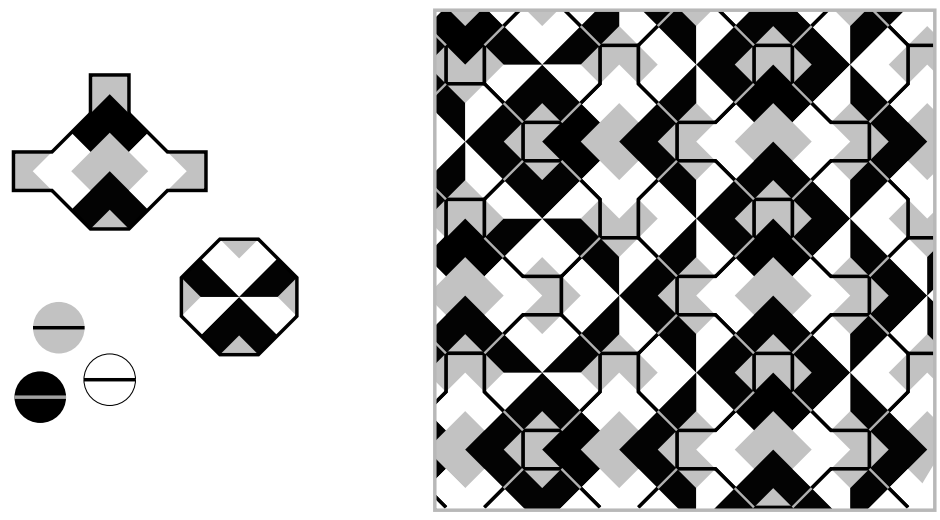

Figure 2: A matching rule tiling

arranged into larger and larger images of inflated and subdivided L-tiles $\Gamma$ as at right in figure 1 (the thicker lines have been added to emphasize the hierarchy). We can then define a global structure- the "substitution tiling" induced by the inflation and division of the tiles.

But L-tiles can tile the plane in myriad ways. Is there a set of local conditions"matching rules" - that Tif satisfied every where $\Gamma$ force the hierarchical structure of the substitution tiling to emerge? One can show that no such rules exist for unmarked L-tiles. However $\Gamma$ we can find a set of marked L-tiles $\Gamma$ and matching rules that force the the original hierarchical structure to emerge. For example $\Gamma$ in figure $2 \Gamma$ we tile with the seven marked L-tiles $\Gamma$ and require that we can cover the plane with overlapping images of the two matching rules at lower left. At right we see a portion of a tiling satisfying these rules $\Gamma$ tiled with marked L's.

As the hierarchical structure of figure 1 is precisely reproduced $\Gamma$ we say the original substitution tiling has been "enforced" by these matching rules. [GS]

That is $\Gamma$ this global structure can be recreated using only locally defined conditions.

Matching rules have been given for a variety of hierarchical tilings with R. Berger's landmark paper [Ber]. R. Robinson gave the first simple example [Rob] $\Gamma$ soon followed by R. Penrose's celebrated rhombs [Pen]. R. Amman [GrSh] $\Gamma$ J. Socolar [Soc] $\Gamma$ L. Danzer [Dan] and others have constructed many beautiful examples. S. Mozes gave rules enforcing one special infinite class of 
substitution tiling [Mos] $\Gamma$ and C. Radin gave rules enforcing the Conway-Radin pinwheel tiling [Rad]. HoweverГno truly general technique had emerged.

Our theorem includes all examples known to the author:

Theorem Every substitution tiling of $\mathrm{E}^{\mathrm{n}}, \mathrm{n}>1$, can be enforced with finite matching rules, subject to a mild condition:

the tiles are required to admit a set of "hereditary edges" such that the substitution tiling is "sibling-edge-to-edge".

Two general methods are known to produce "aperiodic tiles" - tiles that admit no periodic tiling: construct matching rules enforcing substitution tilings; or construct matching rules enforcing a "quasiperiodic" tiling- tilings derived as slices through higher dimensional lattices ([dBr][TSen] $).{ }^{2}$

Our theorem immediately gives an infinite collection of corollaries: any substitution tiling satisfying our technical condition yields a forced aperiodic tiling of the plane. ${ }^{3}$

Le T.T.Q. has recently given a theorem similar to ours $\Gamma$ for quasiperiodic tilings [Le].

A fuller introduction to substitution tilings and many issues of technical or historical interest has been relegated to Appendix A.

The author thanks David MolnarTthe participants of the Geometry Seminar

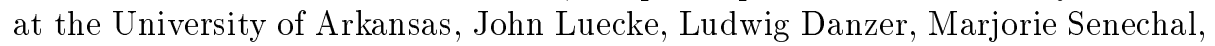
Lorenzo Sadun and Charles RadinГeach for useful discussions at various points in the preparation of this paper.

In Section 0.1 we give a quick expository summary of the techniques involved.

In Section 1 we will establish the setting $\Gamma$ defining basic terms such as "tile" $\Gamma$

\footnotetext{
${ }^{2}$ Exactly one other class of forced aperiodic tilings is known, found by P. Schmitt and altered by J.H. Conway and L. Danzer [Dan]. These tilings of $\mathrm{E}^{3}$ are not isotropic.

${ }^{3}$ Our construction produces aperiodic tiles even if the original substitution tiling is periodic; this is because we specifically enforce a hierarchical structure in the tiling.
} 
"substitution" and "enforcement".

In Section 2 we will select vertices and edgesTand carefully define our "labels" names of elements in structures we draw from the substitution tiling. Our main structures will be "skeletons" and "vertex-wires".

In Section $3 \Gamma$ we describe how to mark tiles with our labels and in Section 3.5 give matching rules.

In Section 4 we prove the matching rules and marked tiles enforce the original substitution tiling.

Appendix A is an expository introduction to substitution tilings.

In Appendix B matching rules are produced for a specific substitution tiling.

In Appendix $\mathrm{C}$ additional formalisms are given.

\subsection{A sketch of the techniques}

In order to sketch the technique of the proof of the theorem we first pause for rough definitions. We will define our terms more precisely in Sections 1 and 2. Our definitions are generally designed to allow enough flexibility to accommodate all historical examples but retain meaning.

For our specific proof of the theorem we take a narrow $\Gamma$ classical setting: "tilings" are to be coverings of $n$-dimensional Euclidean space $E^{n}$ by congruences of a finite set of "prototiles" - marked compact subsets of $E^{n}$. The images of the prototiles under congruences are to be called "tiles"; we require the tiles in a tiling to have disjoint interiors.

A "matching rule tiling" $\left(\mathcal{M} \Gamma \mathcal{T}^{\prime}\right)$ is the set of all tilings by prototiles $\mathcal{T}^{\prime} \Gamma$ that satisfy some local rules $\mathcal{M}$ that specify allowed bounded configurations.

Given a set of prototiles $\mathcal{T} \Gamma$ a "substitution acting on the prototiles" is an expanding linear map $\sigma$ (called an "inflation" or "similarity") on $E^{\text {n }} \Gamma$ such that for each prototile $\mathrm{A} \Gamma \sigma(\mathrm{A})$ is the union of a set $\mathrm{A}^{+}$of "daughter" tiles with disjoint interiors. Thus $\sigma$ may also be thought of as an "inflate and subdivide" operation on configurations of tiles. We take care to require that $\sigma$ can be iterated; any configuration congruent to some $\sigma^{k}(\mathrm{~A}) \Gamma$ for some prototile $\mathrm{A} \Gamma$ we call a "supertile". The specific substitutions are encoded in a set $\mathcal{S}$ of images of the prototiles in the inflated prototiles. 
A "substitution tiling" $(\mathcal{T} \Gamma \sigma \mathcal{S})$ is the set of tilings by "polyhedral" prototiles $\mathcal{T}$ such that any bounded subset of the tiling appears in some supertile given by the substitution defined through $\sigma$ and $\mathcal{S}$.

To define the "enforcement of substitution tiling by matching rules" we must define a "labeling" of a substitution tiling; essentiallyГthis formally allows one to mark information concerning the hierarchy on the supertiles. Then a matching rule tiling "enforces" a substitution tiling if and only if it reproduces this labeling.

The "edges" of a n-dimensional prototile are polyhedral subsets of the $(n-1)$ dimensional facets of the polyhedron underlying the prototile.

A set of edges is "hereditary" if: for any edge $e$ of any prototile $\mathrm{A} \Gamma \sigma^{n}(e)$ is exactly tiled by edges of the prototiles in $\mathrm{A}^{+} \Gamma$ and every $k$-facet $\Gamma 0 \leq \kappa \leq(\mathrm{n}-2)$ of any edge is tiled by $k$-facets of any prototiles in $\sigma^{n}(\mathrm{~A})$ to which it is incident.

A substitution tiling $\Gamma$ with edges defined $\Gamma$ is "sibling edge-to-edge" if for each $\mathrm{B}, \mathrm{C} \in \mathrm{A}^{+} \Gamma$ if an edge $e$ of $\mathrm{B}$ is incident to $\mathrm{C}$ in $\sigma(\mathrm{A}) \Gamma$ then the edge is exactly coincident to some edge $f$ of $\mathrm{C} \Gamma$ and every $k$-facet $\Gamma 0 \leq \kappa \leq(\mathrm{n}-2)$ of $e$ is coincident to a $k$-facet of $f$.

The condition in our theorem is actually that given a set of prototiles and a substitution we are able to define a set of edges that is hereditary and renders the tiling sibling edge-to-edge. This act of defining is made weaker by allowing different edges for each image of the prototiles in the substitution rules.

Naively Tone might expect such edges might always be found-indeed this is so for all examples known to the author at this moment $\Gamma$ although a counterexample may not be difficult to find. The conditions are indeed mild: one can always find edges for the images of the prototiles in the substitutions such that the tiling is sibling edge-to-edge. And one can always take as edges the vertices of the polyhedra underlying the prototiles; these will always be hereditary edges if the prototiles are convex. In a vast collection of examplesTone can find edges satisfying both criteria.

We now describe our techniques. We begin with a substitution tiling $(\mathcal{T}\lceil\bar{F} \mathcal{S})$. We intend for the tiles to organize themselves into larger and larger supertiles -inflations of the original tiles - further and further up the hierarchy. Each $n$-level supertile congruent to $\Gamma$ say $\Gamma \sigma^{(n-1)}(\mathrm{A}) \Gamma \mathrm{A} \in \mathcal{T}$ is to lie in a $(n+1)$-level 
supertile congruent to $\sigma^{n}\left(\mathrm{~A}^{-}\right) \Gamma$ where $A^{-}=\left\{\mathrm{B} \in \mathcal{T} \mid \mathrm{A} \in \mathrm{B}^{+}\right\}$. The essential information associated with each supertile is its own shape and position in the next level of the hierarchy.

Much of the construction given here is foreshadowed in [Moz] $\Gamma$ in which S. Mozes gives matching rules enforcing substitution tilings in which the tiles are all rectangular blocks. [Moz] uses two key observations: each supertile needs only to know its ancestry only a finite number of generations back $\Gamma$ and each supertile should be combinatorially active at only a few key sites.

This information needs to be consistent across the supertile $\Gamma$ needs to be manifest at a few key points on the boundary of the supertileГand any neighborhood in the tiling must contain only a finite amount of information.

We can use the combinatorial structure of our addressing scheme as the basis for a "labeling" of $(\mathcal{T} \Gamma \sigma \Gamma \mathcal{S})$ : this labeling will encode "skeletons" and "wires" to compare and transport information across supertiles. We will define finite classes of "labels" - combinatorial encodings of these mechanisms; the labeling will consist of marking the tiling with these labels.

We will define the elements of our labeling in in Section 2. In Section 3 we derive tiles and matching rules from the local structure of the labeling $\Gamma$ and in Section 4 show these force the hierarchy to organize.

Because we define our structures on inflated prototiles they will be available $\Gamma$ scaled upГon every supertile.

Note we are selecting these structures. We do not assume the "nice" choices are being made. There is thus still ample room to find elegant constructions in more specific cases.

Each $n$-level supertile will consist of $(n-1)$-level supertiles held together by an $n$-level "skeleton" $\Gamma$ defined shortlyГof edges for the parent supertile. The essential information defining this supertile is conveyed in a "packet" of labels along this skeleton. That is Tour matching rules will assure that we have identified each supertile's intended position with respect to its parent with respect to a few recent ancestral supertiles.

A skeleton will be loose and floppy $\Gamma$ a locally defined topological object $\Gamma$ combinatorial in nature $\Gamma$ on top of which is encoded information concerning 
the role of the supertile in the hierarchy. Supertiles are geometrically rigid $\Gamma$ and combinatorially inert. Skeletons provide combinatorial cohesion; supertiles provide geometrical rigidity. Together they force the hierarchy to emerge.

Matching rules at its vertices ensure the skeleton is formed correctly; matching rules at certain "sites" ensure that an $n$-level skeleton correctly meets its descendant $(n-1)$-level skeletons and its parent $(n+1)$-level skeleton.

In the lower left of figure 3 [the substitution for the pinwheel tiling [Rad] is shown; above and to the right skeletons for three generations of supertile are shown. Note the sites $\Gamma$ shown as half circles $\Gamma$ connecting the skeletons of child to parent.

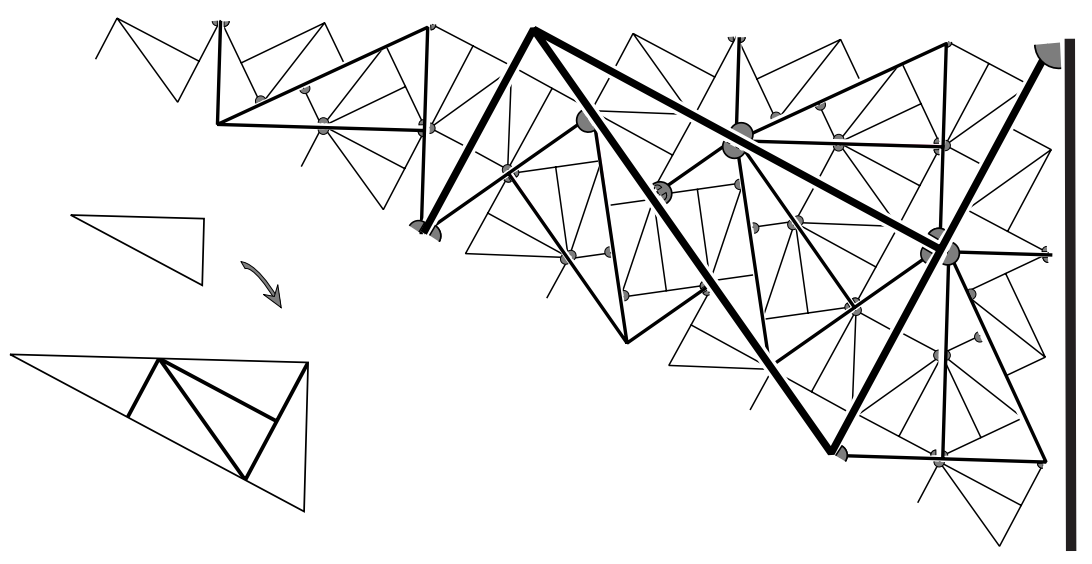

Figure 3: Skeletons

As a technical point Tto ensure that skeletons are connected $\Gamma$ that a supertile's skeleton meets each of the supertile's childrenTand that sites can be chosen $\Gamma$ we allow an $n$-level skeleton to enter lower level supertiles (cf. figure 12). However $\Gamma$ for any substitution tiling we find a constant $\kappa$ so that all $n$-level skeletons include only edges of level at least $(n-\kappa)$ and less than $n .^{4}$ Thus skeletons might overlap but only to a bounded depth.

Each $n$-level supertile $\Gamma$ and skeleton $\Gamma$ is associated with its "address" $\mathrm{X}_{\kappa} \mathrm{X}_{(\kappa-1)} \ldots \mathrm{X}_{1} \Gamma$ relative to its $(n+\kappa-1)$-level ancestor. These digits are in $\mathcal{S}$. Each edge $\Gamma$ vertex $\Gamma$ and site in a skeleton carries the supertile's address and its own label relative

\footnotetext{
${ }^{4}$ In fact, though, in most well known examples, we can take $\kappa=1$. In this case, especially when $\mathrm{n}=2$, the construction simplifies enormously.
} 
to the skeleton in classes defined throughout Section 2. As an edge or vertex might belong to many skeletons $\Gamma$ we may encode many such pairs; however the total information at any point in a tiling will be bounded.

We need a supertile to "know" where certain vertices-"terminals" - are; these vertices are endpoints of the supertile's parent's edges. That is $\Gamma$ if the supertile is of level $n$ Tthe terminals are endpoints of $n$-level edges in the boundary of the supertile. We can hook the terminals into the supertile's skeleton $\Gamma$ if they meet lower level edges in the interior of the supertile (such terminals are "endovertices"). Alas $\Gamma$ this is not often the case and we must introduce another devicewe link certain terminals ("mesovertices") to the skeleton through a series of lower level supertiles. Such a series is a "vertex wire". A supertile may thus carry $\mathrm{Tfor}$ certain of its vertices $\Gamma$ certain information associated with some higher level supertile.

In figure 2.3 vertex wires are shown for three vertices on the pinwheel prototile (the vertex in the middle of the large edge is not really necessary in the actual enforcement of the pinwheel tiling but gives a more interesting vertex wire to illustrate). To the left the three vertices are illustrated; in the middle the wires is drawn as it appears in the tiling- a nested sequence of supertiles converging to the vertices; on the right the wires are drawn as they are abstractly represented-sequences of tiles. The vertex on the left of the prototile does not need a vertex wire; it is incident to the tile's skeleton.

A schematic of all the various structures we will exploit is shown in figure 5 . The bulk of the construction will be an algorithm to encode these structures in our sets of labels Tour packets and tiles.

Once these mechanisms are set $u p \Gamma$ the actual proof that they succeed in enforcing the hierarchical structure is relatively simple. This is necessarily so $\Gamma$ since we cannot rely on combinatorial arguments specific to a given set of tiles. Here is a very quick sketch of the proof of the matching rules:

We derive a labeling of $(\mathcal{T} \Gamma \sigma \Gamma \mathcal{S}) \Gamma$ and from this construct a matching rule tiling $\left(\mathcal{M} \Gamma \mathcal{T}^{\prime}\right)$. A "well-formed supertile" is a configuration of tiles in $\left(\mathcal{M} \Gamma \mathcal{T}^{\prime}\right)$ that is essentially a labeled supertile in $(\mathcal{T} \Gamma \sigma \Gamma \mathcal{S})$.

Once everything is prepared we induct: if every tile in our matching rule tiling $\left(\mathcal{M} \Gamma \mathcal{T}^{\prime}\right)$ lies in a well-formed supertile of level $n \Gamma$ we show every tile must lie in a well-formed supertile of level $(n+1)$. A well-formed supertile has only a few relevant properties: it is clearly marked with its address $X_{\kappa} X_{(\kappa-1)} \ldots X_{1}$ 

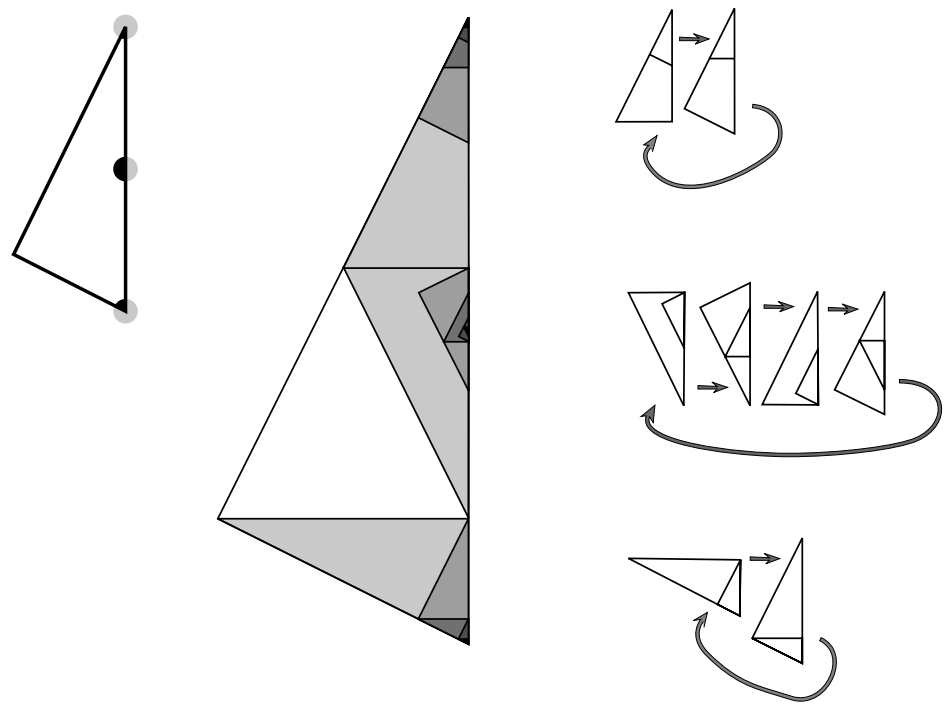

Figure 4: Vertex wires

\section{structures in supertile}
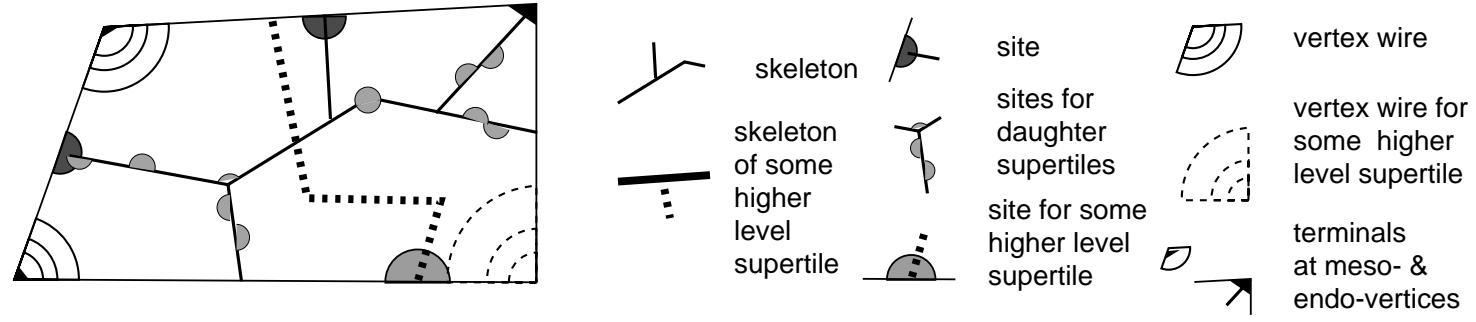

Figure 5: Structures in a supertile 
at the sites on its boundary and at its terminals $\Gamma$ it is actually congruent to $\sigma^{n}\left(\mathrm{X}_{1}\right)$ Tand it is already anticipating any information it must carry for higher level supertiles (that is $\Gamma$ it has clearly marked channels in which higher level skeletons or vertex wires will run).

In figure 6 एwe see in the upper center a schematic of such a $\sigma^{n}\left(\mathrm{X}_{1}\right)$; the terminals $\Gamma$ sites and points at which a higher level skeleton enters the supertile have already be fixedГand are marked with little gray flags. At these points $\Gamma$ all information concerning the supertile itself is determined and consistent; information concerning higher level structures may be present $\Gamma$ but is not yet corroborated nor necessarily consistent.

Then (i) the skeleton of the parent emerges at the sites $\Gamma$ propagating in a uncontrolled but locally well-formed manner along the boundary of the supertile $\Gamma$ until (ii) terminating at the terminals. As edges meet Tinformation concerning the parent supertile begins to be corroborated. Because the tiling is sibling edge-to-edge $\Gamma$ the terminals must be incident to edges that seem to be edges for neighboring sibling supertiles.

As these neighboring edges propagate (iii) sites for sibling supertiles must have been present Talthough initially it may not be clear that the sibling supertiles are the right size or in the right position. However (iv) again the vertex wires come to the rescue; that the tiling is sibling edge-to-edge ensures that the wires of siblings must meet $\Gamma$ and so fixing the siblings adjacent to our original supertile. We can then fix siblings adjacent to these $\Gamma$ and so forth $\Gamma$ until the geometry of the entire parent supertile has been fixed.

Finally because the parent supertile's skeleton is connected $\Gamma$ all information concerning the parent's alleged role in the hierarchy is consistently represented across the entire parent supertile. Thus Tevery tile in every tiling in $\left(\mathcal{M} T^{\prime}\right)$ lies in a well-formed supertile of level $(n+1)$.

The full proof is not much longer; however precisely defining the structures we need requires extreme care. 
Want: $\sigma^{\mathrm{n}}(\mathrm{A})$

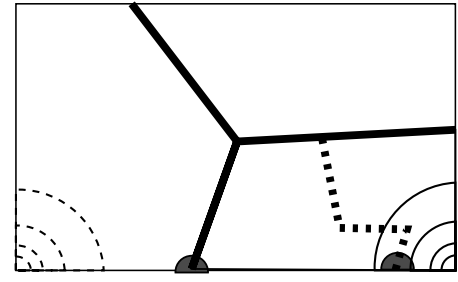

(ii)

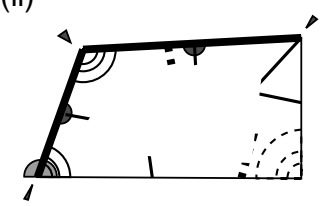

(iii) have: $\sigma^{(n-1)}(B), B$ in $A+$

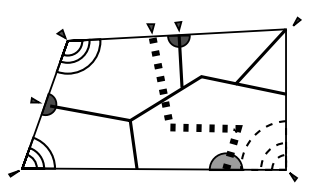

(i)

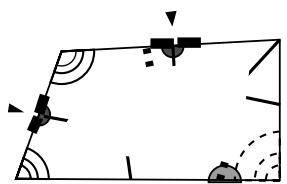

(iv)
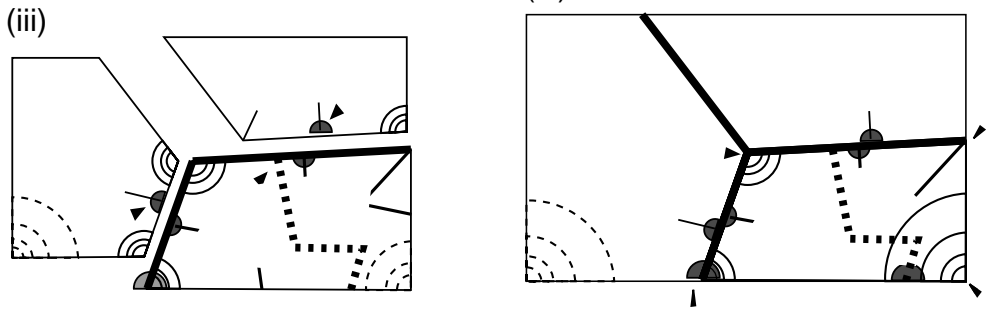

Figure 6: A sketch of the proof of that matching rules succeed

\section{Definitions}

We carefully define a narrow $\Gamma$ classical setting. The construction is mostly combinatorial in nature howeverTand most of these definitions can be expanded in a variety of ways.

In Appendix $\mathrm{C} \Gamma$ we give rather technical definitions of marked prototiles $\Gamma$ matching rules etc. that are essentially equivalent to those we give here but that firmly rest on set theoretic statements.

\subsection{Tiles, matching rules, matching rule tilings}

Let $\mathrm{N}$ be the natural numbers $\Gamma\{1,2 \ldots\}$. We take for our space and congruences $\Gamma$ $\mathrm{n}$-dimensional Euclidean space $\mathrm{E}^{\mathrm{n}} \Gamma 1<\mathrm{n} \in \mathrm{N}$. $\mathcal{G}$ will be the set of Euclidean isometries on $\mathrm{E}^{\mathrm{n}}$.

A prototile $\mathrm{A}$ is a $\mathrm{n}$-dimensional compact set in $\mathrm{E}^{\mathrm{n}} \Gamma$ perhaps marked with some combinatorial information; it is understood that any image of any point in a marked prototile is also marked. ${ }^{5}$

\footnotetext{
${ }^{5}$ In Appendix $\mathrm{C}$ we define these markings through maps $\mu$ to some set $\mathcal{X}$ of combinatorial information
} 
A tile is a congruent image $\mathrm{BA} \Gamma \mathrm{B} \in \mathcal{G}$ of a prototile $\mathrm{A}$.

A tiling $\cup \mathrm{B}_{i} \mathrm{~A}_{i}$ of $X \subset \mathrm{E}^{\mathrm{n}}$ by prototiles $\mathcal{T}$ satisfies: $X=\cup \mathrm{B}_{i} \mathrm{~A}_{i}$ Tand for $i \neq j \Gamma$ $\mathrm{B}_{i} \mathrm{~A}_{i}$ and $\mathrm{B}_{j} \mathrm{~A}_{j}$ have disjoint interiors.

If we do not specify $X$ Twe assume $X=E^{n}$.

A set of tilings of $X$ by prototiles $\mathcal{T}$ Tperhaps with further restrictions $\Gamma$ is a species of tilings.

"Configurations" are often defined as a tiling of some compact $X \Gamma$ but for later convenience we take: A configuration is a subset $\mathrm{D} \subset \cup \mathrm{B}_{i} \mathrm{~A}_{i}$ of points in a tiling.

A finite set $\mathcal{M}$ of matching rules for a tiling of $X$ with prototiles $\mathcal{T}$ is a finite algorithm that examines any bounded configuration in the tiling and determines in a finite number of steps whether the configuration is permitted or not permitted. In particular $\Gamma$ we can choose rules that lock tiles in specific positions to each other or that allow some slipping of tiles past one another (with or without comparing information across such lines of slipping) $\Gamma$ or combinations of these. ${ }^{6}$

A tiling $\cup \mathrm{B}_{i} \mathrm{~A}_{i}$ satisfies a set $\mathcal{M}$ of matching rules if and only if every configuration is permitted.

Note that $\Gamma$ in a sense $\Gamma$ requiring the tiles to fit together with no gaps or overlapping is a matching rule already built into the definition of a tiling $\Gamma$ and turns out to be sufficient for our construction.

A matching rule tiling $\left(\mathcal{M} \Gamma \mathcal{T}^{\prime}\right) \Gamma$ is the species of tilings of $E^{n} \Gamma$ with prototiles $\mathcal{T}^{\prime}$ that satisfy matching rules $\mathcal{M}$.

For example $\Gamma$ in figure $2 \Gamma$ we have two tiles in $T^{\prime}$ marked with colors Black, Gray, and White. Our set of matching rules simply requires that the colors match on the boundaries of neighboring tiles. The image on the right of figure 2 satisfies these matching rules.

\section{$1.2 \quad$ A similarity $\sigma$ and substitutions $\mathcal{S}$}

A similarity will be an expanding linear map acting on $E^{n}$; that is $\Gamma$ a linear map under which all distances increase. Let $\sigma$ be a similarity on $\mathrm{E}^{\mathrm{n}} \Gamma$ with center of dilation at an oriented and fixed origin.

\footnotetext{
${ }^{6}$ In Appendix C, this algorithm is described as checking to see if a tiling can be covered by some finite collection of configurations.
} 
A polyhedron is a complex $\Lambda$ such that: $\Lambda$ is a connected compact n-manifold embedded in $\mathrm{E}^{\mathrm{n}}$ and each $k$-cell $(k$-facet) $\Gamma 0 \leq k \leq \mathrm{n} \Gamma$ of $\Lambda$ is a connected $k$ manifold in the exterior of $\Lambda$ that lies in a $k$-plane in $\mathrm{E}^{\mathrm{n}}$; we require the set of facets of a polyhedron to be finite. The terms "vertex" and "edge" will have special technical meanings relative to this complex $\Gamma$ described below in Section 1.5. We require $\mathrm{n}>1$ henceforth.

Let $\mathcal{T}$ be a finite set of marked polyhedra in $\mathrm{E}^{\mathrm{n}}$; these have a fixed position and orientation with respect to the origin (fixed point) of this map $\sigma$. With no loss of generality $\Gamma$ we may assume the union of 1-facets of any polyhedron in $\mathcal{T}$ is connected. (The 1 -facets will allow us to ensure a well-formed supertile (Section 1.3) has a well-defined orientation.)

We essentially just inflate a tile by some similarity and subdivide the image into congruences of our prototiles to define substitutions $\sigma^{\prime}: \mathcal{T} \rightarrow\left\{\cup \mathrm{C}_{i} \mathrm{~B}_{i}\right\} \Gamma$ a map from the prototiles to tilings such that

$\sigma^{\prime}(\mathrm{A})$ is a tiling $\cup \mathrm{C}_{i} \mathrm{~B}_{i}$ of $\sigma(\mathrm{A})$

and for each of the $\mathrm{C}_{i}$ Tthere is a $\mathrm{C}_{i}^{\prime} \in \mathcal{G}$ with $\sigma \mathrm{C}_{i}=\mathrm{C}_{i}^{\prime} \sigma$

(that is $\Gamma$ if $\cup \mathrm{C}_{i} \mathrm{~B}_{i}$ is a tiling of $\sigma(\mathrm{A}) \Gamma$ then $\cup \mathrm{BC}_{i} \mathrm{~B}_{i}$ will be a tiling of $\sigma(\mathrm{BA})$ ).

The last condition ensures we may repeatedly substitute: for $A \in \mathcal{T}$ define $\left(\sigma^{\prime}\right)^{2}(\mathrm{~A})=\sigma^{\prime}\left(\cup \mathrm{C}_{i} \mathrm{~B}_{i}\right)=\cup \mathrm{C}_{i}^{\prime} \sigma^{\prime}\left(\mathrm{B}_{i}\right)$; we thus may generate larger and larger supertiles $\sigma^{n}(\mathrm{~A})$ of tiling.

That the map is expanding ensures that all $k$ dimensional facets $\Gamma 0<k \leq \mathrm{n}$ of every prototile are eventually subdivided $\Gamma$ for each is bounded and falls into one of only finitely many congruence classes.

To ensure that every tile is really used $\Gamma$ we require $\Gamma$ given $\mathcal{T} \Gamma \sigma$ and $\mathcal{S} \Gamma$ that for each $\mathrm{A} \in \mathcal{T}$ there exists $n \in \mathrm{N}, \mathrm{B} \in \mathcal{T}, \mathrm{C} \in \mathcal{G}$ such that $\mathrm{CA} \subset \sigma^{n}(\mathrm{~B})$.

The level of the supertile $\sigma^{n}(\mathrm{~A})$ is $n$. We also will call the congruent images $\mathrm{B} \sigma^{n}(\mathrm{~A})$ of supertiles $\Gamma$ supertiles as well $\Gamma$ and usually will take $\sigma^{n}(\mathrm{~A})$ to mean any congruent image of $\sigma^{n}(\mathrm{~A})$.

Note we often use $\mathrm{n}$ for dimensionTbut $n$ for level.

Given a supertile $\sigma^{k}(\mathrm{~A})=\cup \sigma^{(k-1)}\left(\mathrm{C}_{i}\left(\mathrm{~B}_{i}\right)\right) \Gamma$ the $\sigma^{(k-1)}\left(\mathrm{C}_{i}\left(\mathrm{~B}_{i}\right)\right)$ are daughter supertiles relative to their parent supertile $\sigma^{k}(\mathrm{~A}) \Gamma$ and are sibling supertiles relative to each other.

When we refer to one supertile inside anotherГe.g. $\sigma^{j}(\mathrm{~B}) \subset \sigma^{n}(\mathrm{~A}) \Gamma$ it will be understood that $\sigma^{j}(\mathrm{~B})$ is a descendant supertile of ancestral $\sigma^{n}(\mathrm{~A})$ 

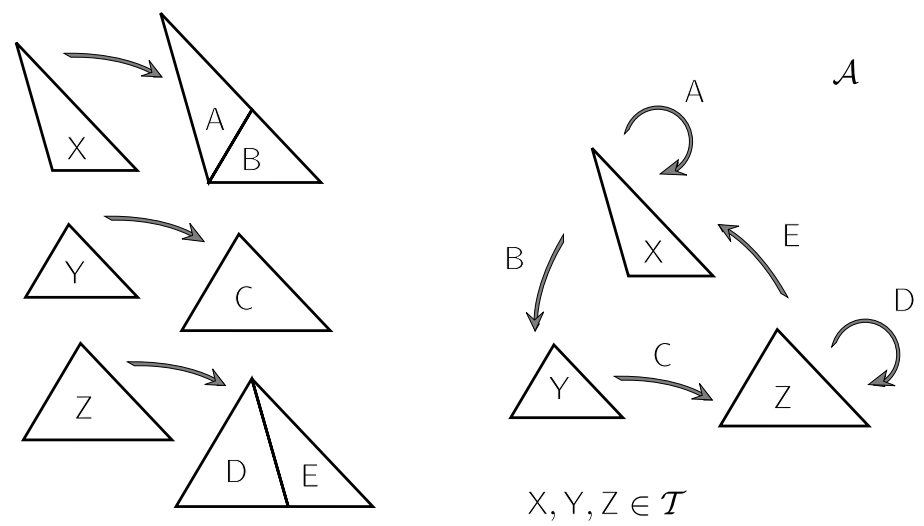

$$
\begin{aligned}
X, Y, Z \in \mathcal{T} & \text { A, B, C, D, E } \in \mathcal{S}
\end{aligned}
$$

Figure 7: A substitution

At left in figure 7 are three prototiles and their substitutions under $\sigma$. The $\mathrm{C}_{i}$ might be regarded as the motions needed to assemble such a diagram.

Note the graph must have a finite number of arrows and nodes because each prototile is compact $\Gamma$ and $|\mathcal{T}| \in \mathrm{N}$. We do not assume that every node can be reached from any other following the arrows Tbut we can be sure that every node is at the head of some arrow (or the corresponding prototile would appear in no supertile). (Incidentally $\mathrm{if}$ every node can be reached from any other $\Gamma$ the substitution tilings must be "repetitive"; cf. [Sen])

For simplicity we coalesce $\sigma$ and $\sigma^{\prime}$ and refer to both the substitution and the similarity as $\sigma$. We hope the context will make the meaning clear. For example $\Gamma$ we may need to refer to the image under $\sigma^{n}$ of some structure $X$ in a prototile $A \Gamma$ relative to the image of the prototile itself. It seems sensible to leave the use of $\sigma$ slightly ambiguous and refer to $\sigma^{n}(X) \subset \sigma^{n}(\mathrm{~A}) \Gamma$ whether the substitution or the similarity has acted on $X$ (figure 8). In particular $\sigma$ almost always will act as a substitution on prototilesTtiles and supertiles; $\sigma$ will almost always act as a similarity on other structures.

Note the requirement that a similar copy of a prototile is congruent to the union of its offspring is very strong indeedГand at first glance seems not to be obeyed in some well known examplesГsuch as the Penrose rhombs (figure 24). However $\Gamma$ these examples can all be recomposed into a tiling with substitution satisfying our requirements; there is undoubtedly a simple sufficient condition (such as "edge-to-edge") for when this can be done. 

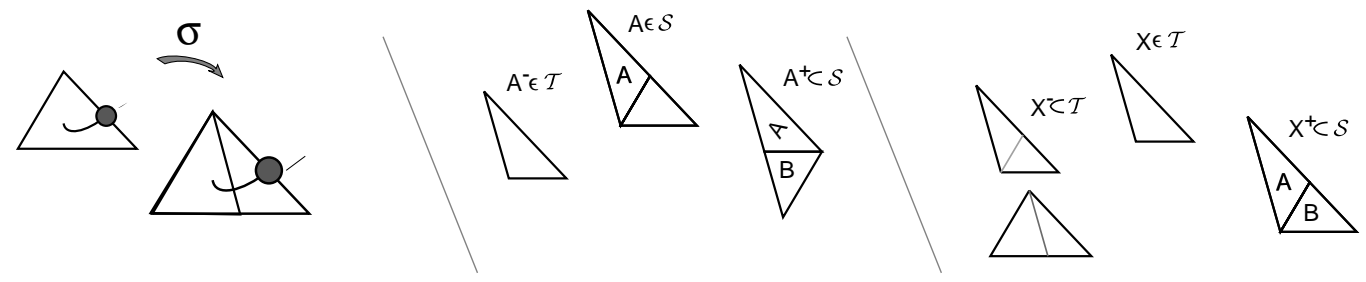

Figure 8: Conventions

It is useful to draw a substitution graph $\mathcal{A} \Gamma$ a directed graph with arrows labeled by congruences and nodes indexed by $\mathcal{T}$ : Each prototile is represented by a node of the graph; if for $A \in \mathcal{T} \Gamma \sigma(\mathrm{A})=\cup\left(\mathrm{C}_{i}\left(\mathrm{~B}_{i}\right)\right) \Gamma$ then the arrows departing the node $A$ are indexed by $\left\{C_{i}\right\}$ and head towards the nodes indexed $\left\{\mathrm{B}_{i}\right\}$. We call the set of labeled arrows $\mathcal{S}$. There is a natural projection from $\mathcal{S}$ to $\mathcal{T}$ : an arrow is mapped to the name of the node at its head. Thus $\Gamma$ a label in $\mathcal{S}$ gives a prototile's name in $\mathcal{T}$ but also provides additional information $\Gamma$ the label of the prototile's parent in $\mathcal{T}$. (A label is simply a name in some defined class of names $\Gamma$ such as $\mathcal{T}$ or $\mathcal{S}$ ). Often we will treat an element of $\mathcal{S}$ as an element of $\mathcal{T}$; consistently we will regard the elements of $\mathcal{S}$ as prototiles carrying the names of their parents in $\mathcal{T}$.

Note that $\mathcal{A}$, in effect, is a finite state automaton. The regular language admitted by the automaton is exactly the set of possible lineages of prototiles as we substitute.

For $\mathrm{A} \in \mathcal{T}$ Tthe collection of possible predecessors (parents) of $\mathrm{A}$ is $\mathrm{A}^{-} \subset \mathcal{T}$; the unique predecessor of $A \in \mathcal{S}$ is $A^{-} \in \mathcal{T}$; for either $A \in \mathcal{T}$ or $A \in \mathcal{S}$ the successors (daughters) of $A$ are $A^{+} \subset \mathcal{S}$. If for $A, B \in \mathcal{S}$ there is $C \in \mathcal{T}$ with $A, B \in C^{+} \Gamma A$ and $B$ are sibling supertiles. We will use other genealogical nomenclature as needed. A substitution graph is illustrated at right in figure 7; only a few of the labels in $\mathcal{S}$ are shown.

Sometimes in designing substitution tilings $\Gamma$ some proto-tiles serve as placeholders and are only subdivided after some finite number of inflations (as in figure 7). That is $\Gamma \mathrm{A}$ is a placeholder if and only if only one arrow departs the node $\mathrm{A}$ in $\mathcal{A}$. Note that because there are only finitely many prototiles and because our substitution is actually inflating our tiles $\Gamma$ we must eventually subdivide our tiles $\Gamma$ as well as any of their $k$-dimensional faces $\Gamma 0<k \leq \mathrm{n}$. 
A substitution tiling $(\mathcal{T} \Gamma \sigma \Gamma \mathcal{S})$ is the species of tilings $\cup \mathrm{B}_{i} \mathrm{~A}_{i}$ by $\mathcal{T}$ such that any bounded configuration in any $\cup B_{i} A_{i}$ is congruent to some configuration in the interior of some supertile $\sigma^{n}(\mathrm{~A}) \Gamma \mathrm{A} \in \mathcal{T} \Gamma n \in \mathrm{N}$.

With no loss of generality we assume every prototile appears in some tiling in $(\mathcal{T} \Gamma \sigma \Gamma \mathcal{S})$.

Lemma 1.1 Let $\cup \mathrm{B}_{i} \mathrm{~A}_{i}$ be a tiling in $(\mathcal{T}, \sigma, \mathcal{S})$. Then for all $\mathrm{B}_{j} \mathrm{~A}_{j}$, for all $n \in \mathrm{N}$, there is a supertile $\sigma^{n}(\mathrm{~A}) \subset \cup \mathrm{B}_{i} \mathrm{~A}_{i}$ with $\mathrm{B}_{j} \mathrm{~A}_{j} \subset \sigma^{n}(\mathrm{~A})$.

That is $\Gamma$ every tile in a tiling in $(\mathcal{T} \Gamma \sigma \Gamma \mathcal{S})$ appears in a supertile in the tiling. This is not quite trivial $\Gamma$ since by the definition of $(\mathcal{T} \Gamma \sigma \Gamma \mathcal{S})$ we only know that an image of any configuration appears in some supertile.

Proof. Let $\mathrm{B}_{j} \mathrm{~A}_{j}$ be a tile in $\cup \mathrm{B}_{i} \mathrm{~A}_{i}$ and induct: When $n=0 \Gamma \mathrm{A}=\mathrm{A}_{j}$ and $\mathrm{B}_{j} \mathrm{~A}_{j}=\sigma^{0}(\mathrm{~A})$ (As will be usual $\Gamma$ we are being casual about specifying that $\sigma^{0}(\mathrm{~A})$ is really a congruence of the supertile). Now suppose that for $(n-1) \Gamma$ there exists $\mathrm{B} \in \mathcal{T}$ with $\sigma^{(n-1)}(\mathrm{B}) \subset \cup \mathrm{B}_{i} \mathrm{~A}_{i}$ with $\mathrm{B}_{j} \mathrm{~A}_{j} \subset \sigma^{(n-1)}(\mathrm{B})$. If there does not exist a $\sigma^{n}(\mathrm{~A})$ with $\sigma^{(n-1)}(\mathrm{B}) \subset \sigma(\mathrm{A}) \Gamma$ then the configuration $\sigma^{(n-1)}(\mathrm{B})$ does not lie in the interior of any supertile.

qed

Lemma 1.2 The union of elements of any nested sequence $\left\{\sigma^{n}\left(\mathrm{~A}_{n}\right)\right\}_{n \in \mathrm{N}}$ of supertiles in a tiling is closed.

Proof Of course each $\sigma^{n}\left(\mathrm{~A}_{n}\right)$ is closed. Let $x$ be any point not in $U \sigma^{n}\left(\mathrm{~A}_{n}\right)$. No tile incident to $x$ could lie in any $\sigma^{n}\left(\mathrm{~A}_{n}\right)$ but $x$ lies in the interior of the union of the tiles incident to $x$. Hence $x$ cannot be a limit point of $\cup \sigma^{n}\left(\mathrm{~A}_{n}\right)$. qed

A tiling $\cup \mathrm{B}_{i} \mathrm{~A}_{i}$ in $(\mathcal{T} \Gamma \sigma \Gamma \mathcal{S})$ has connected hierarchy if and only if for every $x, y \in \cup \mathrm{B}_{i} \mathrm{~A}_{i}$ there exists some supertile $\sigma^{n}(\mathrm{~A})$ such that $x, y \subset \sigma^{n}(\mathrm{~A}) \subset \cup \mathrm{B}_{i} \mathrm{~A}_{i}$.

Lemma 1.3 If a tiling has connected hierarchy then for each point $x$ in the tiling, there exists an $N \in \mathrm{N}$ such that for all $n>N$ there exists an $\mathrm{A} \in \mathcal{T}$ with $x$ in the interior of $\sigma^{n}(\mathrm{~A})$.

Proof Assume there exists a point $x$ in a tiling with connected hierarchy such that for all $n \in \mathrm{N} \Gamma$ there is no A with $x$ in the interior of $\sigma^{n}(\mathrm{~A})$. Now for each $n \Gamma x$ is in some supertile $\sigma^{n}\left(\mathrm{~A}_{n}\right)$ (by Lemma 1.1); we may take these as nested by the proof of that lemma. Now there is a point $y$ in the interior of a tile incident to $x$ with $y \notin \cup \sigma^{n}\left(\mathrm{~A}_{n}\right)$. Let $y^{\prime}$ be a point in the interior of $A_{0}$. By the 
definition of connected hierarchyTthere is some supertile $\operatorname{sigma}^{m}(A)$ containing both $y, y^{\prime}$ Tbut this is a contradiction $\Gamma$ since $y$ is in the interior of each $\mathrm{A}_{n}$ and so belongs to no other supertiles $\Gamma$ but $y^{\prime} \notin \cup \mathrm{A}_{n}$.

qed

\subsection{Enforcement}

We now define "enforcement" of a substitution tiling by matching rules. An equivalent $\Gamma$ more clearly rigorous definition is given in Appendix $\mathrm{C}$.

A labeling $\lambda$ of a substitution tiling $(\mathcal{T} \Gamma \sigma \Gamma \mathcal{S})$ is an algorithm for uniquely marking every tiling in $(\mathcal{T} \Gamma \sigma \Gamma \mathcal{S}) \Gamma$ such that the original markings of the prototiles $\mathcal{T}$ are unambiguously visible $\Gamma$ and such that for any supertile $\sigma^{n}(\mathrm{~A}) \Gamma$ there are only finitely many ways its images in the tilings in $(\mathcal{T} \Gamma \sigma \Gamma \mathcal{S})$ will be marked under $\lambda$.

For example $\Gamma$ in figure $2 \Gamma$ a labeling is given for the $L$-tilings; if one looks closely of only three ways.

Note that this definition is global: the labeling algorithm requires examining the entire infinite tiling $\Gamma$ at least to label some points. For example $\Gamma$ one may

need to decide $\Gamma$ for a given edge in a tiling $\Gamma$ what is the highest level supertile with that edge on its boundary.

Once we have a labeling for $(\mathcal{T} \Gamma \sigma \Gamma \mathcal{S}) \Gamma$ let $\lambda\left(\sigma^{n}(\mathrm{~A})\right)$ be the finite collection of labelings of the supertile $\sigma^{n}(\mathrm{~A})$ and define a well-formed supertile of level $n$ in $\left(\mathcal{M} \Gamma \mathcal{T}^{\prime}\right)$ to be a configuration $X$ of tiles in $\mathcal{T}^{\prime} \Gamma$ satisfying $\mathcal{M} \Gamma$ such that there exist $\mathrm{A} \in \mathcal{T} \Gamma \mathrm{B} \in \mathcal{G} \Gamma$ and $\lambda^{\prime} \in \lambda\left(\sigma^{n}(\mathrm{~A})\right)$ such that all the markings of $\lambda^{\prime} \sigma^{n}(\mathrm{~A})$ coincide with the markings of the tiles in $\mathrm{B} X$.

For example in figure $2 \Gamma$ the well formed supertiles are the pieces of the new tiles that lie in labeled inflated $L$-tiles.

A matching rule tiling $\left(\mathcal{M} \Gamma \mathcal{T}^{\prime}\right)$ enforces a substitution tiling $(\mathcal{T} \Gamma \sigma \Gamma \mathcal{S})$ if and only if one can define a labeling on $(\mathcal{T} \Gamma \sigma \Gamma \mathcal{S})$ such that for every $n \in \mathrm{N} \Gamma$ every point in the interior of any tile in any tiling in $\left(\mathcal{M} \Gamma \mathcal{T}^{\prime}\right)$ lies in a unique well-formed supertile of level $n$.

A substitution tiling $(\mathcal{T} \Gamma \sigma \Gamma \mathcal{S})$ is enforced by finite matching rules if and only if there is a finite set of matching rules $\mathcal{M} \Gamma$ and a finite set $\mathcal{T}^{\prime}$ such that $(\mathcal{M} \Gamma$ $\left.\mathcal{T}^{\prime}\right)$ enforces $(\mathcal{T} \Gamma \sigma \Gamma \mathcal{S})$.

In effect $\Gamma$ we have defined enforcement as being able to parse the matching 
rule tiling into supertiles. Our definition is effectively no stronger or weaker than other definitions known to the author. In particularTsee Lemma C.3.

\subsection{Addresses}

A string is a well-formed list of digits in $\mathcal{S}$. An address is a string drawn from a regular language given by the substitution graph $\mathcal{A}$ (Section 1.2); that is addresses can be viewed as directed paths in $\mathcal{A}$. Addresses are to be read from left to right as the directed path is followed from node to node in $\mathcal{A}$. We allow these paths to be finite $\Gamma$ infinite-to-the-right $\Gamma$ infinite-to-the-left $\Gamma$ or infinite inboth-sides; and we require the appearance of exactly one decimal point $\bullet$ in an address.

The $\bullet$ specifies the "scale" at which we are viewing these addresses.

We wish to assign addresses to tilings; we begin with addresses for inflations of a single tile. So assume the origin is in a fixed orientation at a fixed point in the interior of some fixed tile $\Gamma$ and take this tile's label $A \in \mathcal{T}$ as the first digit to the left of the decimal point $\bullet$. Now this tiling was the result of some action under the substitution system and so our tile at the origin is contained in some supertile that is the image of a prototile under a single substitution. We specify the label in $\mathcal{T}$ of this parent supertile and our original digit now is written in $\mathcal{S}$. Note that since for $\mathrm{A} \in \mathcal{T} \Gamma\left|\mathrm{A}^{-}\right|$may be greater than one $\Gamma$ this gives us some choice at each step of the construction.

In turn $\Gamma$ we give the label in $\mathcal{T}$ of the parent's parent $\Gamma$ taking ever larger configurations $\Gamma$ adding to the left of our address digits that correspond to the labels of ancestors of our original tile $A$. At every finite stage we have an address $\mathrm{X}_{k} \mathrm{X}_{(k-1)} \ldots \mathrm{X}_{2} \mathrm{~A} \bullet$ where $\mathrm{A} \Gamma \mathrm{X}_{(i<k)} \in \mathcal{S} \Gamma \mathrm{X}_{k} \in \mathcal{T} \Gamma \mathrm{X}_{2}=\mathrm{A}^{-} \Gamma \mathrm{X}_{j}=\mathrm{X}_{(j-1)}^{-} \Gamma j \leq k$ (We can take an extra leading $\operatorname{digit} \Gamma \mathrm{X}_{(k+1)}=X_{k}^{-} \in \mathcal{T}$ ).

This address serves an $k$-level supertile $\Gamma$ giving its similarity class $\sigma^{(k-1)}\left(\mathrm{X}_{k}\right)$ and its position and orientation with respect to the origin of the plane.

An infinite-level supertile $\cup \sigma^{k}\left(\mathrm{X}_{k}\right)$ with specified position and orientation with respect to some origin is thus represented by an infinite-to-the-left address $\ldots \mathrm{X}_{k} \ldots \mathrm{X}_{1} \bullet$ where $\mathrm{X}_{j}=\mathrm{X}_{(j-1)}^{-} \Gamma j>1$; Note the collection of these infinite-level supertiles is uncountable $\Gamma$ and includes some partial tilings of the plane. By Lemma subtech $\Gamma$ the infinite-level supertiles are closed.

If we next ignore the origin $\Gamma$ and consider two infinite-level supertiles to be equivalent if there is a congruence taking one to the other $\Gamma$ two infinite-to-theleft addresses ... $\mathrm{X}_{k} \ldots \mathrm{X}_{1} \bullet$ and $\ldots \mathrm{Y}_{k} \ldots \mathrm{Y}_{1} \bullet$ give the same infinite-level supertile if 


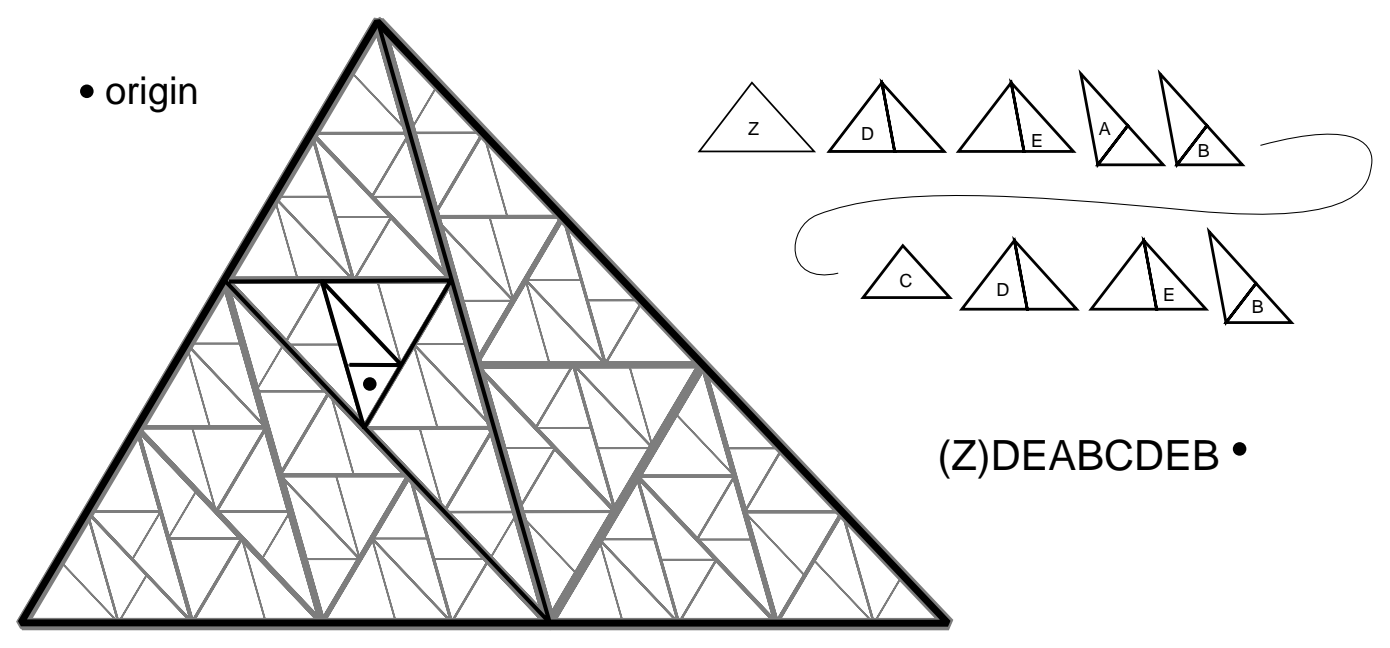

Figure 9: an address of a supertile relative to a fixed origin

and only if there is an natural number $i$ such that $\mathrm{X}_{j}=\mathrm{Y}_{j}$ for all $j \geq i$; that is if and only if they are identical on the left after some finite number of digits. We will take such classes of addresses as the address of an infinite-level supertile.

Note that the tilings in $(\mathcal{T} \Gamma \sigma \Gamma \mathcal{S})$ either are infinite-level patches $\Gamma$ or are two or more infinite level patches joined along $(n-k)$-dimensional half-planes emanating from some $k$-plane $\Gamma 0 \leq k \leq \mathrm{n}$. Any such lower dimensional planes and half planes are infinite fault-lines.

Infinite-to-the-right addresses $A \bullet \ldots$ are also useful $\Gamma$ describing the location of a point in a tile. (We use that $\mathrm{E}^{\mathrm{n}}$ is complete and the similarity $\sigma^{-1}$ shrinks all distances). That is $\Gamma$ a point with address $A \bullet X_{-1} \ldots X_{-n} \ldots$ lies in the image under $\sigma^{-n}$ of $\mathrm{X}_{-n} \subset \sigma^{n}(\mathrm{~A})$. In this context $\Gamma$ an address is rational if of the form $A \bullet X Y Y Y \ldots$ where $X, Y$ are finite addresses-the initial string and repeating string respectively. We can continue to associate addresses with a variety of other structures

Thus an address structure is thus a well-defined, algorithmic coding of a hierarchical structure residing in some geometric space. In some sense $\Gamma$ the full substitution structure is a lexicon of a regular language encoded in the geometry of $E^{n}$.

These address structures have a much richer structure than we have outlined 
for our present needs. ${ }^{7}$

Let $\mathcal{A}^{-}$be the set of all infinite-to-the-left addresses. For any infinite level supertile $S$ एwe have defined a map $\lambda_{\mathcal{A}}$ from the tiles in an infinite level supertile to infinite-to-the-left addresses as described above.

\subsection{Vertices and edges}

We will use the terms "edges" and "vertices" in very technical senses. Note we strongly use $\mathrm{n}>1$.

An edge of a prototile $\mathrm{A}$ in a substitution tiling $(\mathcal{T} \Gamma \sigma \Gamma \mathcal{S})$ is a polyhedral subset $e$ of some $(\mathrm{n}-1)$-facet of the polyhedron underlying the tile $\Gamma$ such that $e$ is the image under the similarity $\sigma^{-k} \Gamma$ for some $k \in \mathrm{N} \Gamma$ of the union of $(\mathrm{n}-1)$ facets in the boundary of the supertile $\sigma^{k}(\mathrm{~A})$. That is $\Gamma$ edges arise by subdividing facets $\Gamma$ and new edges can be derived by subdividing previously defined edges. Note facets are edges.

A set of edges for a prototile is a set of edges that cover the boundary of the prototile and have disjoint interiors. We will repeatedly redefine the elements of these sets of edges throughout the construction: in Section 2.1.1 we will begin with a set $\mathcal{E}$ edges that are among the facets of our prototiles; in Section 2.1.4 we will inflate and subdivide these to produce a class $\mathcal{E}^{\prime}$; finally in Section $2.2 .2 \Gamma$ a third class $\mathcal{E}^{\prime \prime}$ will be derived from $\mathcal{E}^{\prime}$.

An edge of a tile is a ( $n-1)$-facet of the tile; an edge of a supertile is the image of the edge of a tile under inflation $\sigma^{n}$; the level of an edge is $n$. Note that each point of an edge of a tile is coincident to one or more other edges $\Gamma$ of neighboring tiles. Finally $\mathrm{T}$ an edge of a tiling is the image of the edge of a supertile in a tiling; note that each edge of a tile in a tiling belongs to exactly one edge of the tiling $\Gamma$ and is coincident to a least one other edge of the tiling. (Our edges are more like "sides of edges" $\Gamma$ in the context of a tiling).

\footnotetext{
${ }^{7}$ Address structures turn out to be very versatile, and can encode virtually all of the structure in the space of substitution tilings. In particular, in this one framework, for a given substitution system acting on tiles, we can encode points in a tile, tiles in a tiling, the space of substitution tilings, the ergodic dynamics of the inflation and substitution maps, natural topologies on the space of tilings, and matching rules between tilings. In addition, they provide a natural extension of symmetry from the realm of groups to that of algorithms - that is, they provide a description of "algorithmic symmetry".
} 
Once we have defined a set of edges of a prototile $\Gamma$ we define the vertices of a prototile to be the points incident to 0-dimensional facets of the edges. Thus the vertices are discrete.

The vertices of a tile $\mathrm{BA} \Gamma \mathrm{B} \in \mathcal{G} \Gamma \mathrm{A} \in \mathcal{T}$ Tare simply the images of the vertices of $A$ under $B$. The vertices of a tiling are the points in the tiling that are vertices of tiles in the tiling; a vertex of a tiling may be coincident to a vertex of all or somerbut at least one of the tiles incident to the vertex. The endpoints of an edge are the vertices of the edge.

A set of edges is hereditary if: for any edge $e$ of any prototile $\mathrm{A} \Gamma \sigma(e) \subset \sigma(\mathrm{A})$ is exactly tiled by edges of the tiles in $\mathrm{A}^{+}$Tand every $k$-facet $\Gamma 0 \leq \kappa \leq(\mathrm{n}-2)$ of $\sigma(e)$ is tiled by $k$-facets of tiles in $\sigma(A)$.

Similarly a set of vertices is hereditary if for every vertex $v$ of prototile AГfor each $B \in \mathrm{A}^{+}$incident to $\sigma(v) \subset \sigma(\mathrm{A}) \Gamma$ there is a vertex of $B$ coincident to $\sigma(v)$.

A substitution tiling $\Gamma$ with edges defined $\Gamma$ is sibling edge-to-edge if for each $\mathrm{B}, \mathrm{C} \in \mathrm{A}^{+}$Tif an edge $e$ of $\mathrm{B}$ is incident to $\mathrm{C}$ in $\sigma(\mathrm{A}) \Gamma$ then $e$ is exactly coincident to some edge $f$ of $C \Gamma$ and every $k$-facet $\Gamma 0 \leq \kappa \leq(\mathrm{n}-2)$ of $e$ is coincident to a $k$-facet of $f$.

A substitution tiling $\Gamma$ with vertices defined $\Gamma$ is sibling vertex-to-vertex if for each $\mathrm{B}, \mathrm{C} \in \mathrm{A}^{+} \Gamma$ if a vertex $v$ of $\mathrm{B}$ is incident to $\mathrm{C}$ in $\sigma(\mathrm{A}) \Gamma$ then $v$ is exactly coincident to some vertex of $C$.

These conditions are indeed mild: one can always find edges for the images of the prototiles in the substitutions such that the tiling is sibling edge-to-edge. And one can always take as edges the edges of the polyhedra underlying the prototiles; these will always be hereditary edges. An example of a tiling not satisfying the condition may not be hard to find ThoweverTespecially for $n>2$.

Lemma 1.4 If a set $\mathcal{E}$ of edges for a substitution tiling is hereditary, [the tiling is sibling-edge-to-edge], then the corresponding vertices $\mathcal{V}$ are hereditary, [the tiling is sibling-vertex-to-vertex]. When $\mathrm{n}=2$, the converse holds as well.

Proof This immediately follows from the definitions.

qed

However $\Gamma$ the converse is probably not true when $n>2$ : there are likely to be tilings that are sibling vertex-to-vertex that are not sibling edge-to-edge. 


\subsection{Epi-, Meso- and Endo- vertices}

We give technical definitions of endovertices $\Gamma$ mesovertices and epivertices:

First for each $\mathrm{A} \in \mathcal{T}$ let $\mathcal{E}(\mathrm{A})$ be $(\mathrm{n}-1)$-facets in the interior of $\sigma(A)$. Note that the elements of $\mathcal{E}(A)$ are edges of the elements of $A^{+}$.

Second by hypotheses $\Gamma$ we can define hereditary vertices $\mathcal{V}(A)$ for each $A \in \mathcal{S} \Gamma$ such that the substitution tiling $\{\mathcal{T}, \sigma, \mathcal{S}\}$ is sibling-vertex-to-vertex. Note that the $\mathcal{V}(A)$ are a set of vertices if we take the $(n-1)$-facets of $A$ as edges for $A$.

Then we say:

$v \in \mathcal{V}(\mathrm{A})$ is an endovertex if and only if: both there is some (minimal) positive integer $\kappa(v)$ such that $\sigma^{\kappa(v)}(v)$ is incident to some edge in the interior of $\sigma^{\kappa(v)}(\mathrm{A})$ (and hence $\sigma^{k}(v)$ incident to some edge in the interior of $\sigma^{k}(\mathrm{~A})$ for all $\left.k \geq \kappa(v)\right) \Gamma$ and also $v$ is incident to some $e \in \mathcal{E}\left(\mathrm{A}^{-}\right)$in $\sigma\left(\mathrm{A}^{-}\right)$.

$v \in \mathcal{V}(\mathrm{A})$ is an mesovertex if and only if: $v$ is incident to some $e \in \mathcal{E}\left(\mathrm{A}^{-}\right)$in $\sigma\left(\mathrm{A}^{-}\right) \Gamma$ but $\sigma^{k}(v)$ is not incident to any edge in the interior of $\sigma^{k}(\mathrm{~A})$ for any $k$.

Finally $v \in \mathcal{V}(\mathrm{A})$ is an epivertex if and only if: $v$ is not incident to any $e \in \mathcal{E}\left(\mathrm{A}^{-}\right)$ in $\sigma\left(\mathrm{A}^{-}\right)$.

Note that if $v \in \mathcal{V}(\mathrm{A})$ is in the interior of $\sigma\left(\mathrm{A}^{-}\right)$Tit is incident to some $e \in \mathcal{E}\left(\mathrm{A}^{-}\right)$ in $\sigma\left(\mathrm{A}^{-}\right)$. The endo- $\Gamma$ meso- and epi- vertices of tiles $\Gamma$ supertiles and tilings are the images of the endo- $\Gamma$ meso- and epi- vertices of prototiles.

Terminals are another name for endo- and meso- vertices.

In figure 10 vertices are shown for the dimer substitution of figure 22 ). Endo- $\Gamma$ meso- and epi- vertices are marked. (Labels in $\mathcal{S}$ and $\mathcal{T}$ have also been assigned.)

Lemma 1.5 If a vertex is a mesovertex for a given n-level supertile, the vertex is not coincident to a mesovertex for any descendant or ancestral supertile. Moreover, the vertex is coincident to an epivertex for every descendant supertile incident to the vertex.

Proof Let $v$ be a mesovertex for some prototile $\mathrm{A} \in \mathcal{S}$.

For every $k \Gamma 0 \leq k \leq n \Gamma \sigma^{n}(v)$ is coincident to some vertex $\sigma^{k}\left(v_{k}\right) \in$ $\sigma^{k}\left(A_{k}\right) \subset \sigma^{n}(\mathrm{~A})$.

Since $v$ is a mesovertex $\Gamma$ no edge meets $\sigma^{n}(v)$ in the interior of $\sigma^{n}(\mathrm{~A})$. Consequently $\Gamma v_{k}$ is an epivertex of $\mathrm{A}_{k}$. 
Second $\Gamma$ suppose $v$ is coincident to some vertex $\sigma^{n}\left(v_{n}\right)$ of some ancestral supertile $\sigma^{n}\left(\mathrm{~A}_{n}\right)$. Because $v$ is a mesovertex $\Gamma v$ is incident to some edge $e \in$ $\sigma\left(A^{-}\right) \subset \sigma^{n}\left(A_{n}\right)$. Thus $\Gamma \sigma^{n}\left(\mathrm{~A}_{n}\right)$ is a mesovertex or endovertex.

$$
\text { qed }
$$

Lemma 1.6 In a tiling in $(T, \sigma, \mathcal{S})$, any vertex $v$ of any prototile $\mathrm{A}$ in the interior of some supertile $\sigma^{m}(\mathrm{~B})$ is either a mesovertex for some supertile contained in $\sigma^{m}(\mathrm{~B})$ and containing $\mathrm{A}$, or for some $n \in \mathrm{N}, \sigma^{n}(v) \subset \sigma^{n}(\mathrm{~A})$ is coincident to an edge in the interior of $\sigma^{n}(\mathrm{~A})$.

Proof Let $B A$ be a tile in the tiling such that $B A$ is in the interior of some supertile. Let $\lambda_{\mathcal{A}^{-}}(\mathrm{BA})=\ldots \mathrm{X}_{n} \ldots \mathrm{X}_{1} \mathrm{~A}$.

Let $v$ be a vertex of prototile A such that for no $n \in \mathrm{N} \Gamma \sigma^{n}(v) \subset \sigma^{n}(\mathrm{~A})$ is coincident to an edge in the interior of $\sigma^{n}(\mathrm{~A})$. Thus $v$ is either a mesovertex or an epivertex of $A$. A itself is a supertile $\Gamma$ so in the first case we are done.

So suppose $v$ is an epivertex of A. Now there exists some $N \Gamma$ such that for all $n>N \Gamma v$ is in the interior of $\sigma^{n}\left(\mathrm{X}_{n}\right)$ and for all $n \leq N \Gamma v$ is on the boundary of $\sigma^{n}\left(\mathrm{X}_{n}\right)$. There exists some maximal $M \leq N$ such that for all $n \leq M \Gamma v$ is on the boundary of $\sigma^{n}\left(\mathrm{X}_{n}\right)$ but not incident to any edge in $\sigma^{n}\left(\mathrm{X}_{n}\right)$. Thus $v$ is a mesovertex or endovertex of $\sigma^{M}\left(\mathrm{X}_{M}\right)$. But for any $n \Gamma \sigma^{n}(v)$ is not coincident to an edge in the interior of $\sigma^{(n+M)}\left(\mathrm{X}_{M}\right)$; thus $v$ is a mesovertex of $\sigma^{n}\left(\mathrm{X}_{N}\right)$. qed

Note that the hypothesis holds for all vertices of tiles in a tiling with connected hierarchy. 


\section{Selecting structures in a substitution tiling}

We now begin to select structures in our substitution tiling $(\mathcal{T} \Gamma \sigma \Gamma \mathcal{S})$ in order to define a labeling (Section 1.3).

There are a few main categories of label:

In Section 1 we described our initial categories $\mathcal{T} \Gamma \mathcal{S}$.

In Section 2.1 we define labels $\Gamma$ in $\mathcal{V} \Gamma \mathcal{V}^{\prime} \Gamma \mathcal{E} \Gamma \mathcal{E}^{\prime} \Gamma$ and $\mathcal{Z} \Gamma$ concerning our first structure $\Gamma$ skeletons. We also choose our important constant $\kappa$.

In Section $2.2 \Gamma$ we construct keys $\Gamma$ labels in $\mathcal{R} \Gamma \mathcal{V}^{\prime \prime}$ and $\mathcal{E}^{\prime \prime}$ that define the role of a supertile in the hierarchy.

In Section 2.3 we construct labels $\mathcal{W}$ concerning our second structure wires $\Gamma$ and wire keys $\mathcal{U}$.

In Section 2.4Twe construct well-formed packets in $\mathcal{Q}$ and $\mathcal{P}$ Tcombinatorially complex combinations of previously defined labels; through our efforts here and in Section 3.3 Wwe will have fairly simple matching rules (Section 3.5).

We begin with a given substitution system $\{\mathcal{T}, \sigma, \mathcal{S}\} \Gamma$ and $\lambda_{\mathcal{A}}$ mapping each tile in each tiling to an infinite-to-the right address. Crucially $\Gamma$ note that even if a tiling admits more than one hierarchy $\Gamma \lambda_{\mathcal{A}}$ fixes a particular hierarchyГand every tile belongs to only one supertile of each level $n$ in this fixed hierarchy.

\subsection{Selecting structures to form skeletons}

\subsubsection{Selecting vertices $\mathcal{V}$ and edges $\mathcal{E}$}

By assumptionTthere is a set of hereditary edges on the prototiles such that the tiling is sibling edge-to-edge. Let $\mathcal{E}_{0}(\mathrm{~B})$ be such edges of $\mathrm{B} \in \mathcal{S}$. For each $\mathrm{A} \in \mathcal{T} \Gamma$ let $\mathcal{E}(\mathrm{A})$ be the edges $\mathcal{E}_{0}(\mathrm{~B}) \Gamma \mathrm{B} \in \mathrm{A}^{+} \Gamma$ that are not contained in the boundary of $\sigma(\mathrm{A})$. By sibling edge-to-edge we can take these edges as occurring in pairs $\Gamma$ $+e,-e$. Let $\mathcal{E}$ be the disjoint union of the $\mathcal{E}(\mathrm{A}) \Gamma \mathrm{A} \in \mathcal{T}$.

Given an $e \in \mathcal{E} \Gamma$ we will take as implicit the element $\mathrm{A}$ in $\mathcal{T}$ such that $e \in$ $\mathcal{E}(\mathrm{A}) \Gamma$ as well as the exact position of $e$ in $\sigma(\mathrm{A})$. Note that in any configuration $\Gamma$ the interiors of any $\sigma^{n}(e) \Gamma \sigma^{m}\left(e^{\prime}\right)$ are $\operatorname{disjoint} \Gamma n \neq m \Gamma e \neq \pm e^{\prime} \Gamma e, e^{\prime} \in \mathcal{E}$.

For $e \in \mathcal{E}$ let $e+=A \in \mathcal{S}$ if $e \in \mathcal{E}_{0}(\mathrm{~A})$ 
Let $\mathcal{V}(\mathrm{A}) \Gamma \mathrm{A} \in \mathcal{S}$ be defined as the vertices of $\mathcal{E}_{0}(\mathrm{~A})$. Note that these are hereditary and the tiling is sibling vertex-to-vertex by Lemma 1.4.

Let $\mathcal{V}$ be the disjoint union of the $\mathcal{V}(\mathrm{A})$ as $\mathrm{A}$ ranges over $\mathcal{S}$; in particularTan element $v$ of $\mathcal{V}$ lies in a unique $\mathcal{V}(\mathrm{A})$.

Given a $v \in \mathcal{V} \Gamma$ we will take as implicit the element $\mathrm{A}$ in $\mathcal{S}$ such that $v \in \mathcal{V}(\mathrm{A}) \Gamma$ as well as the exact position of $v$ on the boundary of A. (This kind of implicitness will be usual as all these lists of labels are made).

In figure $10 \Gamma \mathcal{V}$ and $\mathcal{E}$ are shown for the dimer substitution of figure 22.
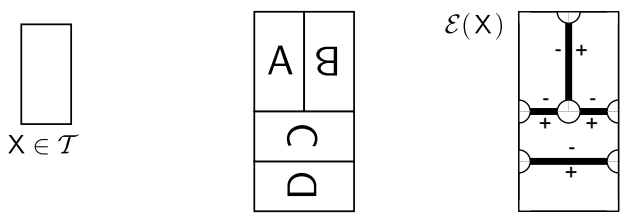

$\mathrm{A}, \mathrm{B}, \mathrm{C}, \mathrm{D} \in \mathcal{S}$

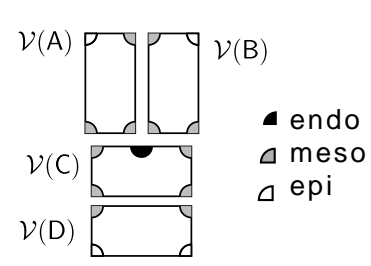

Figure 10: Edges $\mathcal{E}$ and vertices $\mathcal{V}$

\subsubsection{Selecting sites $\mathcal{Z}$}

For each $A \in \mathcal{S}$ we choose a number of sites that will link the skeleton of any supertile congruent to $\sigma^{n}(\mathrm{~A})$ to the skeleton of the parent supertile congruent to $\sigma^{(n+1)}\left(\mathrm{A}^{-}\right)$at edges congruent to $\sigma^{n}(\mathcal{E}(\mathrm{A}))$.

For each $e \in \mathcal{E} \Gamma$ choose a natural number $\kappa(e)$ such that the supertile $\sigma^{\kappa(e)}(e+)$ contains an edge with an endpoint on $\sigma^{\kappa(e)}(e) \subset \sigma^{(\kappa(e)+1)}\left((e+)^{-}\right)$.

For each $\mathrm{A} \in \mathcal{S}$ Гwe can choose a point $\mathcal{Z}_{e}$ on each edge $e \in \mathcal{E}(\mathrm{A})$ meeting $+e$ such that there is an edge in the interior of $\sigma^{\kappa}(+e)$ with one end at $\sigma^{k}\left(\mathcal{Z}_{e}\right) \subset$ $\sigma^{\kappa}(e) \subset \sigma^{(\kappa+1)}(\mathrm{A})$. We take these points as sites for the prototile $+e \in \mathcal{S}$; in a supertile congruent to $\sigma^{n}(\mathrm{~A}) \Gamma \mathrm{A} \in \mathcal{S} \Gamma$ each point corresponding to a $\sigma^{n}\left(\mathcal{Z}_{e}\right) \Gamma$ $+e=\mathrm{A} \in \mathcal{S} \Gamma$ is to be a site for the supertile .

In practice one might eliminate redundancies. First $\Gamma$ sites as defined may lie at the endpoint of an edge. Thus two sites for a supertile may lie at the same point. We simply coalesce these into one site. Second $\Gamma$ endovertices make excellent sites $\Gamma$ since we will connect these to a supertile's skeleton anyway. These steps do nothing for the proof but ease one's burden in practice. Let 
$\mathcal{Z}(\mathrm{A})$ be the collection of sites serving $\mathrm{A}$.

We come to a technicality: $\mathcal{E}\left(\mathrm{A}^{-}\right)$may be empty - this occurs if and only if $A$ is a placeholder (Section 1.2). Sites need to be more carefully designed for such $A \in \mathcal{S}$ :

Because $\sigma$ expands all distances $\Gamma$ we must eventually subdivide the facets of our tiles; if $\mathrm{A}^{-}$is a placeholder $\Gamma$ there is an integer $j \geq 0$ and $\mathrm{B} \in \mathcal{S}$ such that: $\sigma^{j}(\mathrm{~B})$ consists only of $\mathrm{A}$ but $\mathcal{E}\left(\mathrm{B}^{-}\right)$is not empty. Then let $(\mathcal{Z}(\mathrm{A}))$ lie at the corresponding sites $\sigma^{j}(\mathcal{Z}(\mathrm{B})) \subset \mathrm{A}$.

\subsubsection{Selecting $\kappa$}

In a sense $\kappa$ is the resolution at which we view the hierarchical structure.

For each $\mathrm{B} \in \mathcal{S}$ choose natural $\kappa(\mathrm{B})$ such that there is a connected collection of edges in $\sigma^{\kappa(\mathrm{B})}(\mathrm{B})$ that

i) contains every $\sigma^{(\kappa(\mathrm{B})-1)}(e)$ for $e \in \mathcal{E}(\mathrm{B}) \Gamma$

ii) meets every $\mathcal{Z}(\mathrm{B})$ on the boundary of $\mathrm{B}$ and

iii) meets every endovertex on the boundary of $B$.

It is worth pointing out that we can indeed find such a $\kappa(\mathrm{B}): \kappa(\mathrm{B})$ is at least the maximum of the $\kappa(v)$ as $v$ ranges over the endovertices of $\mathrm{B}$ and of the $\kappa(e) \Gamma e \in \mathcal{E}(\mathrm{B})$. To ensure that we can find a connected collection of edges $\Gamma$ we may have to take further substitutions. Eventually however $\Gamma$ such a $\kappa(\mathrm{B})$ can be found: $\sigma$ expands all distances $\Gamma$ all facets are bounded and fall into finite congruence classes; hence $\sigma$ must eventually subdivide every $k$-facet $\Gamma<k \leq \mathrm{n}$.

MoreoverГany $k>\kappa(\mathrm{B})$ will suffice as well $\Gamma$ since edges are hereditary.

Take $\kappa$ to be the maximum of these $\kappa(B)$. In practice $\Gamma \kappa$ is often rather low; in fact $\Gamma$ many well known examples have $\kappa=1$ and very few have $\kappa>2$. For example $\Gamma$ the Conway-Radin pinwheel $[\operatorname{Rad}] \Gamma$ the Sphinx [Godr] $\Gamma$ and the systems studied by Mozes [Moz] each allow $\kappa=1$ Tas do most of the examples in Appendix A. In the current incarnation of our construction $\Gamma$ the $L$-tiling requires $\kappa=2$ leading to a set of markings that is far from optimal [GS].

One can easily construct examples requiring arbitrarily large $\kappa \Gamma$ such as Sadun's generalized pinwheel tilings [Sad]. Often a specific construction of matching rules can be finessed somewhat - in particular note that our bound $\kappa$ is the maximum of many other bounds each of which will play specific roles in the construction.

In figure $11 \Gamma$ sites and $\kappa$ have been chosen for the dimer tiling of figure 22 . Note that we require $\kappa=2 \Gamma$ both to find an appropriate site on one of the edges 
abutting prototile $A \in \mathcal{S}$ (defined in figure 10) $\Gamma$ and so that we have a connected collection of edges that includes the image $\mathcal{E}(\mathrm{B})$ in each $\mathrm{B} \in \mathcal{S}$.

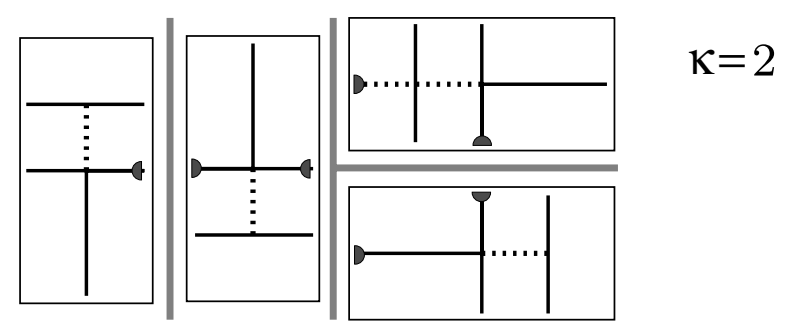

Figure 11: Sites $\Gamma \kappa$ and skeletons for a dimer tiling

\subsubsection{Selecting vertices $\mathcal{V}^{\prime}$ and edges $\mathcal{E}^{\prime}$ of skeletons}

Lemma 2.1 Given $(\mathcal{T}, \sigma, \mathcal{S})$, using hereditary edges $\mathcal{E}$, there exists $\kappa \in \mathrm{N}$, and sites $\mathcal{Z}$ such that

for each $\mathrm{A} \in \mathcal{S}$ we may choose a skeleton of $j$-level edges, $0 \leq j<\kappa$, $E_{\mathrm{A}} \subset \sigma^{\kappa}(\mathrm{A})$ such that

i) $\cup_{e \in E(\mathrm{~A})} \sigma^{(\kappa(e)-1)} \subset E_{\mathrm{A}}$;

ii) $E_{\mathrm{A}}$ is connected;

iii) $\sigma^{\kappa}(\mathcal{Z}(\mathrm{A})) \subset E_{\mathrm{A}}$;

iv) $E_{\mathrm{A}}$ includes the endovertices of $\sigma^{\kappa}(\mathrm{A})$.

Proof This immediately follows the definitions and existence of $\kappa$ and $\mathcal{Z}$. qed

We now subdivide our edges in $E_{\mathrm{A}}$ into edges $\mathcal{E}^{\prime}(\mathrm{A})$ of the supertiles $\sigma^{(\kappa-1)}(\mathrm{B}) \Gamma$ $\mathrm{B} \in \mathrm{A}^{+}$in order to describe the structure of this skeleton $E_{\mathrm{A}}$. We require that:

Each edge $\sigma^{j}(e) \Gamma 0 \leq j<\kappa$ contained in $E_{\mathrm{A}}$ is tiled with edges in $\mathcal{E}^{\prime}(\mathrm{A})$; and any facet of an edge in $\mathcal{E}^{\prime}(A)$ is a facet of any edge in $\mathcal{E}^{\prime}(A)$ to which it is incident.

We strongly require hereditary edges to ensure such an $\mathcal{E}^{\prime}(A)$ exists: we could simply take appropriate $(\mathrm{n}-1)$-facets in $\sigma^{\kappa}(\mathrm{A})$; typically $\mathrm{C}$ this is choice far from optimal.

We define the set $\mathcal{V}^{\prime}(\mathrm{A})$ of vertices of $E_{\mathrm{A}}$ to be the set of points coincident to 
vertices of the edges in $\mathcal{E}^{\prime} \Gamma$ union the points coincident to $\sigma^{(\kappa-1)}(z) \Gamma z \in \mathcal{Z}(\mathrm{B}) \subset$ $E_{\mathrm{A}}, \mathrm{B} \in \mathrm{A}^{+}$Tunion the points coincident to $\sigma^{\kappa}(\mathcal{V}(\mathrm{A})$.

Suppose an edge $f \in \mathcal{E}^{\prime}(\mathrm{A})$ was derived from some $\sigma^{j}(e) \Gamma e \in \mathcal{E}$; then the level of $\sigma^{n}(f)$ will be $(n+j)$.

Let $\mathcal{V}^{\prime} \Gamma \mathcal{E}^{\prime}$ be the disjoint union of the $\mathcal{V}^{\prime}(\mathrm{A}) \Gamma \mathcal{E}^{\prime}(\mathrm{A})$ over $\mathcal{S}$.

Given a $v \in \mathcal{V}^{\prime}$ Tit is to be implicit for which $\mathrm{A} \in \mathcal{S}$ that $v \in \mathcal{V}^{\prime}(\mathrm{A})$ and the exact position of $v$ in $\sigma^{\kappa}(\mathrm{A})$. Given an $e \in \mathcal{E}^{\prime} \Gamma$ it is to be implicit for which $\mathrm{A} \in \mathcal{S}$ that $e \in \mathcal{E}^{\prime}(\mathrm{A})$ and the exact position of $e$ in $\sigma^{\kappa}(\mathrm{A})$.

Note that for any vertex $v \in \mathcal{V}^{\prime}(\mathrm{A}) \Gamma v \subset \sigma^{\kappa}(\mathrm{A}) \Gamma$ if $v$ is incident to some $\sigma^{j}(\mathrm{~B}) \Gamma$ $B \in \mathcal{S} \Gamma 0 \leq j<\kappa \Gamma v$ does not need to be coincident to some vertex $\sigma^{j}(w)$ of $\sigma^{j}(\mathrm{~B}) \Gamma w \in \mathcal{V}(\mathrm{B})$.

In figure 12 the skeletons of three generations of supertiles in the dimer tiling of figure 22 are shown. Note skeletons frequently overlap of parent and child.

\section{$2.2 \quad$ Keys}

Roughly keys encode position and role relative to a few levels of the hierarchy.

\subsubsection{Supertile keys $\mathcal{R}$}

Edges of tiles in tilings in $(\mathcal{T} \Gamma \sigma \Gamma \mathcal{S})$ might serve up to $\kappa$ distinct skeletons.

We specify the ways this might occur. For each $A \in \mathcal{S}$ let $\mathcal{R}(\mathrm{A})$ be the collection of all supertile keys $\Gamma$ finite addresses $\mathrm{X}_{\kappa} \ldots \mathrm{X}_{1}$ where $x_{j} \in \mathcal{S} \Gamma \mathrm{X}_{(j+1)}=\mathrm{X}_{j}^{-}$ for $j<\kappa$ Tand $\mathrm{X}_{1}=\mathrm{A}$. Note that such a address could have been thought of as having $\kappa+1$ elements $\Gamma$ for $\mathrm{X}_{\kappa}$ implicitly specifies the label of its parent in $\mathcal{T}$. Let $\mathcal{R}$ be the disjoint union of these $\mathcal{R}(\mathrm{A})$ over $\mathcal{S}$. (To facilitate a certain technical point below $\Gamma$ we also include an extra null label in $\mathcal{R}$; this label contains no combinatorial information.) Now a given $\mathrm{R}=\mathrm{X}_{\kappa} \ldots \mathrm{A} \in \mathcal{R}(\mathrm{A})$ exactly specifies all skeletons for all ancestral supertiles that pass through any supertile congruent to $\sigma^{n}(\mathrm{~A})$ with address ...R...•.

A label in $\mathcal{R}$ is the crucial information, then, that we will ensure that every supertile carries.

We begin defining maps $\lambda_{*}$ to structures in a generic tiling with connected 

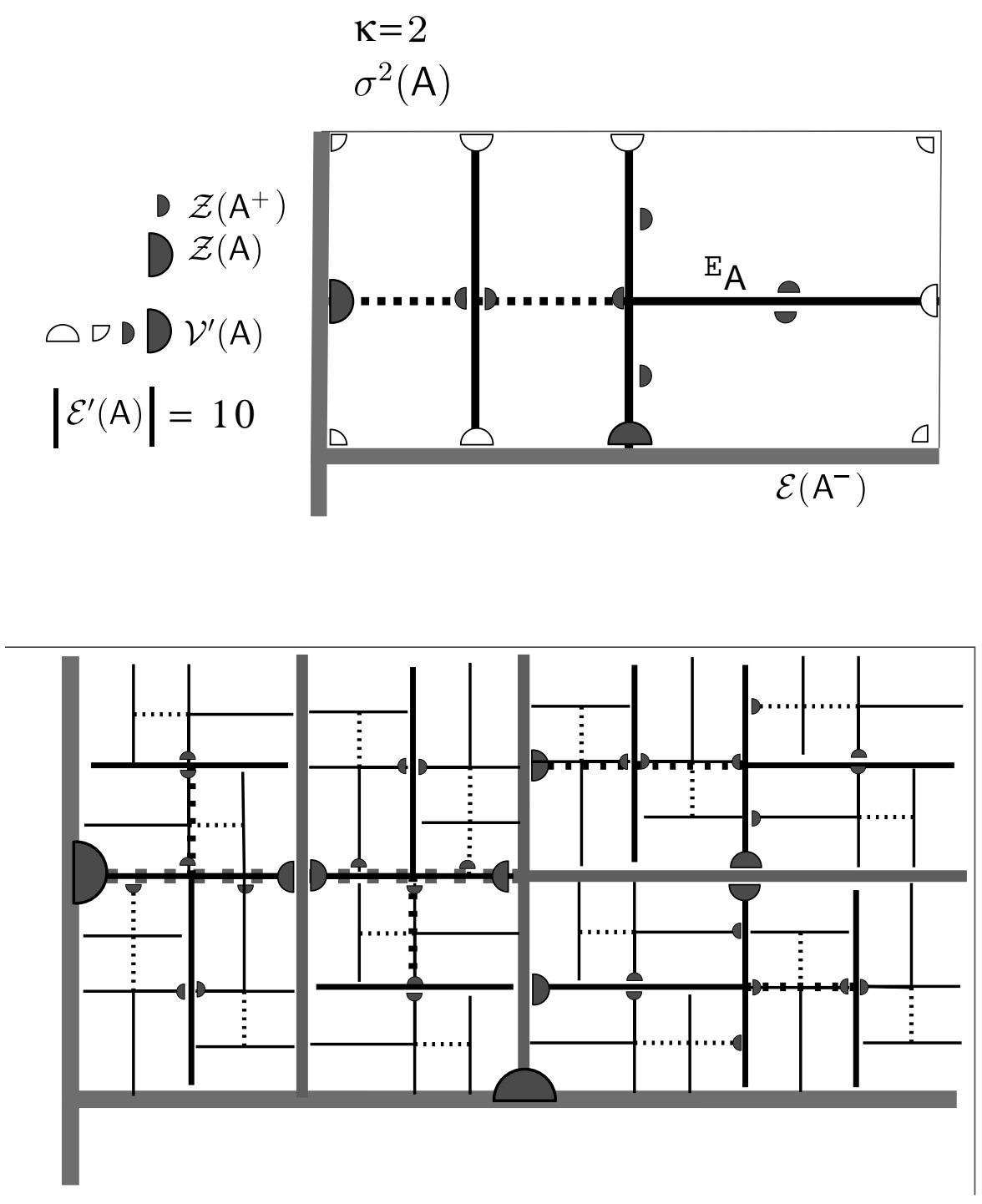

Figure 12: Skeletons of three generations of dimer 
hierarchy. This will eventually be the basis for a labeling (Section 1.3) of the substitution tiling.

Lemma 2.2 Let $\cup \mathrm{B}_{i} \mathrm{~A}_{i}$ be a tiling in $(\mathcal{T}, \sigma, \mathcal{S})$, with $\lambda_{\mathcal{A}^{-}}$(Section 1.4) chosen for $\cup \mathrm{B}_{i} \mathrm{~A}_{i}$. Then there exists a map $\lambda_{\mathcal{R}}:\left\{\mathrm{B} \sigma^{n}(\mathrm{~A}) \mid \mathrm{B} \in \mathcal{G}, n \in \mathrm{N}, \mathrm{A} \in\right.$ $\left.\mathcal{S}, \mathrm{B} \sigma^{n}(\mathrm{~A}) \subset \cup \mathrm{B}_{i} \mathrm{~A}_{i}\right\} \rightarrow \mathcal{R}$ such that if $\lambda_{\mathcal{R}}\left(\sigma^{n}(\mathrm{~A})\right)=\mathrm{X}_{\kappa} \ldots \mathrm{X}_{1}$ then $\mathrm{X}_{1}=\mathrm{A}$ and for all $\mathrm{B} \in \mathrm{A}^{+}, \lambda_{\mathcal{R}}\left(\sigma^{(n-1)}(\mathrm{B}) \subset \sigma^{n}(\mathrm{~A})\right)=\mathrm{X}_{(\kappa-1)} \ldots \mathrm{X}_{1} \mathrm{~B}$, and if $\lambda_{\mathcal{A}^{-}}\left(\mathrm{B}_{i} \mathrm{~A}_{i}\right)=$ $\ldots \mathrm{X}_{\kappa} \ldots \mathrm{A}_{i} \bullet, \lambda_{\mathcal{R}}\left(\mathrm{B}_{i} \mathrm{~A}_{i}\right)=\mathrm{X}_{\kappa} \ldots \mathrm{A}_{i}$.

Proof In $\sigma^{n}(\mathrm{~A}) \in \cup \mathrm{B}_{i} \mathrm{~A}_{i} \Gamma$ for any $\mathrm{B}_{i} \mathrm{~A}_{i}, \mathrm{~B}_{j} \mathrm{~A}_{j} \subset \sigma^{n}(\mathrm{~A}) \Gamma$ if $\lambda_{\mathcal{A}^{-}}\left(\mathrm{B}_{i} \mathrm{~A}_{i}\right)=\ldots \mathrm{X}_{k} \ldots \mathrm{A}_{i} \bullet$ and $\lambda_{\mathcal{A}^{-}}\left(\mathrm{B}_{j} \mathrm{~A}_{j}\right)=\ldots \mathrm{Y}_{k} \ldots \mathrm{A}_{j} \bullet$ then for all $k \geq n \Gamma \mathrm{X}_{k}=\mathrm{Y}_{k}$. Take $\lambda_{\mathcal{R}}\left(\sigma^{n}(\mathrm{~A})\right)=$ $\mathrm{X}_{(\kappa+n-1)} \ldots \mathrm{X}_{n}$. Clearly this meets the conditions of the lemma. qed

\subsubsection{Edge keys $\mathcal{E}^{\prime \prime}$}

We again redefine edges and vertices relative to the supertile keys. Take $R=$ $\mathrm{X}_{\kappa} \ldots \mathrm{X}_{1} \in \mathcal{R}$. Recall that for $\mathrm{A} \in \mathcal{S} \Gamma \mathcal{E}^{\prime}(\mathrm{A})$ and $\mathcal{V}^{\prime}(\mathrm{A})$ are defined within $\sigma^{\kappa}(\mathrm{A})$.

We now subdivide the edges $\mathcal{E}\left(\mathrm{X}_{1}\right)$ to produce a new set of edges $\mathcal{E}^{\prime \prime}(\mathrm{R})$ for the prototiles $B \subset \sigma\left(\mathrm{X}_{1}\right) \Gamma \mathrm{B} \in \mathrm{X}_{1}^{+}$:

Let $\mathcal{E}_{\kappa}$ be the set of facets of the tiles in $\sigma^{\kappa}\left(\mathrm{X}_{1}\right)$ such that each facet in $\mathcal{E}_{\kappa}$, lies in some $\sigma^{(\kappa-1)}(e) \Gamma e \in \mathcal{E}\left(\mathrm{X}_{1}\right)$. Then $\Gamma$ first: the edges $\mathcal{E}^{\prime \prime}(\mathrm{R})$ in $\sigma\left(\mathrm{X}_{1}\right)$ will be the union of facets of the form $\sigma^{-\kappa}(f) \Gamma f \in \mathcal{E}_{\kappa}$ (thus each edge $e \in \mathcal{E}\left(\mathrm{X}_{1}\right)$ is tiled with edges in $\left.\mathcal{E}^{\prime \prime}(\mathrm{R})\right)$.

Second we require of $\mathcal{E}^{\prime \prime}(\mathrm{R})$ that every facet of $\sigma^{(j-\kappa)}(e) \subset \sigma\left(\mathrm{X}_{1}\right) \subset \sigma^{j}\left(\mathrm{X}_{j}\right) \Gamma$ $0 \leq j \leq \kappa \Gamma e \in \mathcal{E}^{\prime}\left(\mathrm{X}_{j}\right) \Gamma \mathrm{X}_{0} \in \mathrm{X}_{1}^{+}$is the union of facets of edges in $\mathcal{E}^{\prime \prime}(\mathrm{R})$.

That is $\Gamma$ we are careful to design structures at every facet at which the overlapping skeletons $\sigma^{(j-\kappa)}\left(E_{\mathrm{X}_{j}}\right) \Gamma 0 \leq j \leq \kappa \Gamma \mathrm{X}_{0} \in \mathrm{X}_{1}^{+}$meet the edges $\mathcal{E}\left(\mathrm{X}_{1}\right)$ in $\sigma\left(\mathrm{X}_{1}\right) \subset \sigma^{\kappa}\left(\mathrm{X}_{\kappa}\right)$.

Note such an $\mathcal{E}^{\prime \prime}(\mathrm{R})$ exists:

A poor choice is simply to take all $(\mathrm{n}-1)$-facets in $\sigma^{-k}\left(\left(\sigma^{\kappa}(\mathrm{A})\right)\right.$ ) (where the $\sigma^{-k}$ is a similarity and the $\sigma^{\kappa}$ is an iterated substitution).

Let $\mathcal{E}^{\prime \prime}$ be the disjoint union of the $\mathcal{E}^{\prime \prime}(\mathrm{R})$ over R.

Lemma 2.3 Any edge $f$ of any tile in the interior of some supertile in a tiling in $(\mathcal{T}, \sigma, \mathcal{S})$, is contained within a unique supertile edge $\sigma^{n}\left(e^{\prime \prime}\right), e^{\prime \prime} \in \mathcal{E}^{\prime \prime}$ or there exists $\left(\mathrm{R}=\ldots \mathrm{X}_{1}\right) \in \mathcal{R}, n<\kappa-1$ such that $f$ is a subset of a tiling by edges $\sigma^{n}\left(e_{i}\right), e_{i} \in \mathcal{E}^{\prime \prime}(\mathrm{R})$. In this latter case, though, there is an $e \in \mathcal{E}\left(\mathrm{X}_{1}\right)$ such that $f, \sigma^{n}\left(e_{i}\right) \subset \sigma^{n}(e)$. 
Note that every edge of every tile in a tiling with connected hierarchy is in the interior of some supertile.

Proof Let $f$ be an edge of a tile $\mathrm{B}_{i} \mathrm{~A}_{i}$ in the interior of some supertile in the tiling. There is a minimal level supertile $\sigma^{n}\left(\mathrm{X}_{1}\right)$ containing this tile such that the interior of $f$ is in the interior of $\sigma^{n}\left(\mathrm{X}_{1}\right)$. Thus $f$ lies on the boundary of $\sigma^{(n-1)}(\mathrm{B})$ for some $\mathrm{B} \in \mathrm{X}_{1}^{+}$and in $\sigma^{n}(e)$ for some $e \in \mathcal{E}\left(\mathrm{X}_{1}\right)$. The elements of $\mathcal{E}^{\prime \prime}$ are the unions of the images under $\sigma^{-\kappa}$ of the edges of tiles; thus $f$ lies in the union of the images under $\sigma^{(n-1+\kappa)}$ of edges of tiles. In particular $\Gamma$ since edges are hereditary $\Gamma$ if $n \geq \kappa-1 \Gamma f$ lies in the image of one edge of one tile and so lies in one $\sigma^{n}\left(e^{\prime \prime}\right)$; otherwise $f$ is a subset of a tiling by edges $\sigma^{n}\left(e_{i}\right) \Gamma$ $e_{i} \in \mathcal{E}^{\prime \prime}(\mathrm{R})$ for some $\mathrm{R} \in \mathcal{R}$. By definition $\Gamma$ each $\sigma^{n}\left(e_{i}\right)$ lies in $\sigma^{n}(e) . \quad$ qed

Lemma 2.4 Any edge $f$ of any tile not in the interior of any supertile in a tiling in $(\mathcal{T}, \sigma, \mathcal{S})$, is contained within a unique infinite fault-line

Proof Let $f$ be such an edge. $f$ lies on the exterior of some tile $\Gamma$ contained in a nested series $\left\{\sigma^{n}\left(\mathrm{~A}_{n}\right)\right\}$ of supertiles; $f$ must lie on the exterior of each $\sigma^{n}\left(\mathrm{~A}_{n}\right)$ and so in the exterior of $\cup \sigma^{n}\left(\mathrm{~A}_{n}\right)$ and hence on an infinite fault-line. qed

Thus we define $\Gamma$ for any tiling $\cup \mathrm{B}_{i} \mathrm{~A}_{i}$ in $(\mathcal{T} \Gamma \sigma \Gamma \mathcal{S}) \Gamma$ with $\mathcal{E}^{\prime \prime}$ derived in Section 2.2.2Гa map $\lambda_{\mathcal{E}^{\prime \prime}}: E \rightarrow \mathcal{E}^{\prime \prime} \cup\{$ null $\}$ where $E$ is the set of all points of all edges of tiles in the tiling: for each $x$ a point in some edge $f$ of some tile in the tilingTif $f$ is in the interior of some supertile ${ }^{2}$ let $\lambda_{\mathcal{E}^{\prime \prime}}(x)$ be the $e^{\prime \prime} \in \mathcal{E}^{\prime \prime}$ or $e_{i} \in \mathcal{E}^{\prime \prime}$ produced by Lemma 2.3 such that $x$ lies in $\sigma^{n}\left(e^{\prime \prime}\right)$ or $\sigma^{n}\left(e_{1}\right)$; otherwise let $\lambda_{\mathcal{E}^{\prime \prime}}(f)=$ null.

\subsubsection{Vertex hulls $\mathcal{V}^{\prime \prime}$}

Take $\mathrm{R}=\mathrm{X}_{\kappa} \ldots \mathrm{X}_{1} \in \mathcal{R} \Gamma$ and let $\mathcal{V}^{\prime \prime}(\mathrm{R})$ be the images of the intersection with $\sigma\left(\mathrm{X}_{1}\right)$ of exceedingly small $\delta$ balls centered at each vertex of $\sigma\left(\mathrm{X}_{1}\right)$ and at the points coincident to endpoints of the elements of $\mathcal{E}^{\prime \prime}(\mathrm{R})$. The elements of $\mathcal{V}^{\prime \prime}$ are called vertex hulls; the

center of a hull in $\mathcal{V}^{\prime \prime}(\mathrm{R})$ is the point at which the $\delta$ ball defining the hull is centered.

Lemma 2.5 The set of centers of the $\mathcal{V}^{\prime \prime}(\mathrm{R})$ is the set of all points $v$ in $\sigma\left(\mathrm{X}_{1}\right)$ such that:

$v$ is an endpoint of of some $\sigma^{(j-\kappa)}(e), e \in \mathcal{E}^{\prime}\left(\mathrm{X}_{j}\right), 1 \leq j \leq \kappa$ with $\sigma^{(j-\kappa)}(v) \in$ $f$ for some $f \in \mathcal{E}\left(\mathrm{X}_{1}\right)$,

or $v=\sigma(w), w \in \mathcal{V}\left(\mathrm{X}_{1}\right)$,

or $\left.v=\sigma^{(}-\kappa\right)(z), z \in \mathcal{Z}(\mathrm{A}), \mathrm{A} \in \mathrm{X}_{1}^{+}$. 
Proof This follows the definitions.

That is $\Gamma$ for $\mathcal{V}^{\prime \prime}$ we take the endpoints of any edges in higher level skeletons meeting the 0 -level edges in $X_{1} \Gamma$ the images of the vertices of $X_{1}$ under $\sigma^{1} \Gamma$ and the images of the sites of the daughter tiles on the edges of $X_{1}$.

Place an arbitrary ordering in $\mathcal{V}^{\prime \prime}$. Now we can assume that the 1 -facets of each edge in $\mathcal{E}^{\prime \prime}$ are connected. Define for each $e \in \mathcal{E}^{\prime \prime}$ the set $\mathcal{F}(e)$ of labels of 1-facets. Each endpoint of an element of $\mathcal{F}(e)$ is associated with a label in $\mathcal{V}^{\prime \prime}$; we associate each label in $\mathcal{F}(e)$ with an arrow pointing from the lower endpoint to the higher. Let $\mathcal{F}$ be the disjoint union over $\mathcal{E}^{\prime \prime}$ of the $\mathcal{F}(e)$.

We mark each hull in $\mathcal{V}^{\prime \prime}$ with information concerning the edges it meets:

First the hull is darkly marked with the positions $\Gamma$ orientations $\Gamma$ labels in $\mathcal{E}^{\prime}\left(\mathrm{X}_{j}\right)$ and the relative level $j$ of the various edges in $\sigma^{(j-\kappa)}(e) \Gamma e \in \mathcal{E}^{\prime}\left(\mathrm{X}_{j}\right) \Gamma$ $1 \leq j \leq \kappa$ incident to the vertex in $\sigma\left(\mathrm{X}_{1}\right) \Gamma$ as well as any edges $\sigma^{(-\kappa)}(e) \Gamma$ $e \in \mathcal{E}^{\prime}(A) \Gamma A \in \mathrm{X}_{1}^{+}$for which $v$ incident to $v$. Note that a darkly marked edge in a vertex hull may end up with many edge markings in $\mathcal{E}^{\prime}$; however $\Gamma$ we take the single edge of lowest relative level as our marking $\Gamma$ for this will be sufficient.

Second $\Gamma$ given $v \in \mathcal{V}^{\prime \prime} \Gamma$ there is some finite $N$ such that if $\sigma^{N}(v)$ is incident to an edge $e$ in $\sigma^{(N+1)}\left(\mathrm{X}_{1}\right) \Gamma$ then for any $n>N \Gamma \sigma^{n}(v)$ is incident to $\sigma^{(n-V+1)}(e)$ in $\sigma^{(n+1)}\left(\mathrm{X}_{1}\right) \Gamma$ because of the finite valence of the vertices in our substitution tiling. That is $\Gamma$ we can lightly mark the positions $\Gamma$ orientations $\Gamma$ and labels in $E^{\prime}$ of all lower level edges that are eventually incident to $v$. We also put a height function on these lightly marked edges: suppose $e \Gamma f$ are lightly marked edges incident to $v$ such that there is some $n$ such that $\sigma^{n}(v)$ is incident to an image of $e$ but not incident to an image of $f$; then $e$ is higher than $f$.

On the 1-facets of all the edges Tlightly marked or darkly marked $\Gamma$ we further mark the vertex hull with appropriate labels and orientations in $\mathcal{F}$. (When $\mathrm{n}=2$ we simply place arrows on the elements of $\mathcal{E}^{\prime \prime}$ ).

Note that this hull will be either an $n$-ball or a sector of a n-ball. Because we have assumed sibling vertex-to-vertex $\Gamma$ the first case always occurs if $v$ is in the interior of $\sigma(\mathrm{A})$; the latter case will occur only on the boundary of $\sigma(\mathrm{A})$. In the latter case define the flat sides of the hull to be image of the boundary of the $\sigma(\mathrm{A})$ on the boundary of the hull (i.e. the planes on which the $\mathrm{n}$-ball was cut to make a sector). (Here sibling vertex-to-vertex is not really being used very strongly; to drop the condition we merely have to delineate a second kind of flat side). 
Given $v \in \mathcal{V}^{\prime \prime}\left(\mathrm{X}_{\kappa} \ldots \mathrm{X}_{1}\right)$ Tits hull will be implicit. The hulls will orient a vertex in the tiling $\Gamma$ showing in which directions edges are expected.

In figure $13 \Gamma$ the construction of $\mathcal{E}^{\prime \prime}(A C)$ and $\mathcal{V}^{\prime \prime}(A C)$ is indicated for the dimer tiling of figure 22 and figure 10. It is important to note that these hulls actually have much more information encoded within them-in particular specific labels associated with these particular spots in the structure. Also light and dark edges are not distinguished in this figure.

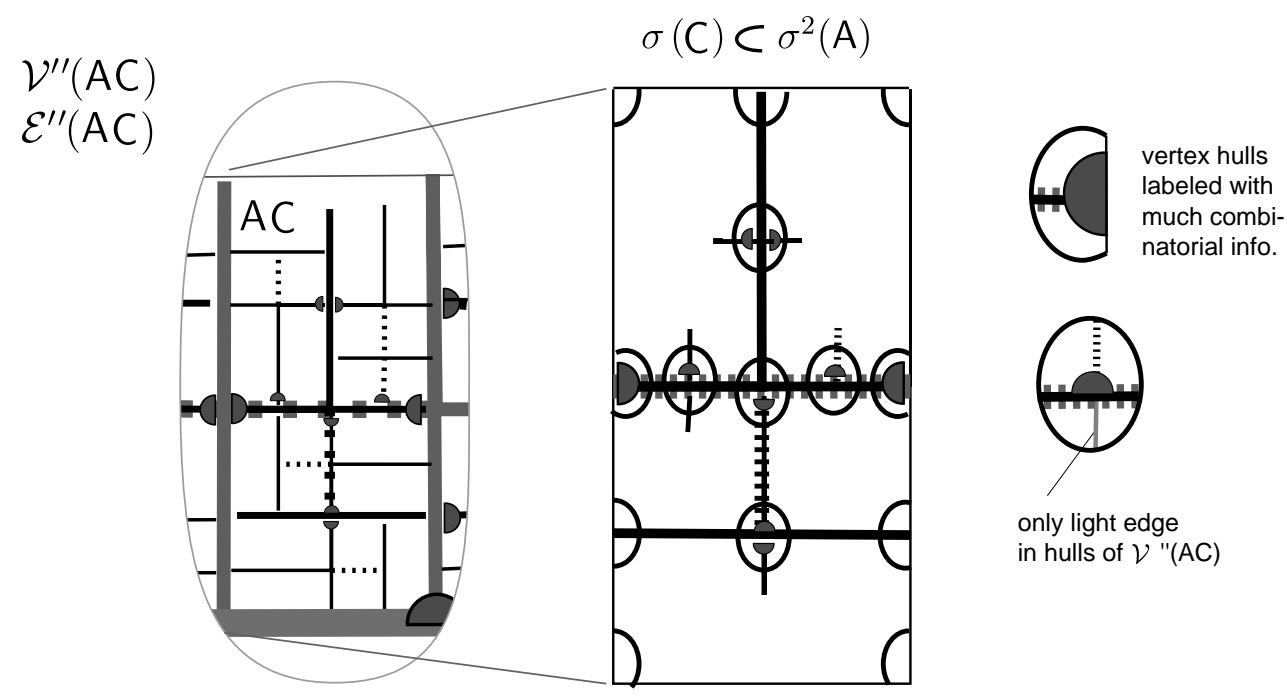

Figure 13: elements of $\mathcal{V}^{\prime \prime} \Gamma \mathcal{E}^{\prime \prime}$ and vertex hulls

Note that $\mathcal{E}^{\prime \prime}\left(\mathrm{X}_{\kappa} \ldots \mathrm{X}_{1}\right) \Gamma \mathcal{V}^{\prime \prime}\left(\mathrm{X}_{\kappa} \ldots \mathrm{X}_{1}\right)$ are defined as structures in $\sigma\left(\mathrm{X}_{1}\right)$. Also note that any edge of level greater than $\kappa$ Tof a tile in a tiling has a unique label in $\mathcal{E}^{\prime \prime}$ (whereas it may have up to $\kappa$ labels in $\mathcal{E}^{\prime}$. Also if the edge is of level less than $\kappa$ The construction of $\mathcal{E}^{\prime \prime}$ may have divided it into some finite number of pieces). We can also mark terminals as appropriate on the vertex hulls $\Gamma$ if they are at the end of a dark edge of relative level-15on the exterior of $\sigma\left(\mathrm{X}_{1}\right)$.

Labels $v \in \mathcal{V}^{\prime \prime}(\mathrm{R}) e \in \mathcal{E}^{\prime \prime}(\mathrm{R}) \Gamma$ with $\mathrm{R}=\mathrm{X}_{\kappa} \ldots \mathrm{X}_{1} \in \mathcal{R} \Gamma$ exactly specify what role $\Gamma$ if any $\Gamma \sigma^{n}(v)$ and $\sigma^{n}(e)$ play in skeletons serving supertiles $\sigma^{(n-1+j)}\left(\mathrm{X}_{j}\right) \Gamma$ $1 \leq j \leq \kappa$; that is $\Gamma$ a edge or vertex label $e \in \mathcal{E}^{\prime \prime}\left(\mathrm{X}_{\kappa} \ldots \mathrm{X}_{1}\right) \Gamma v \in \mathcal{V}^{\prime \prime}\left(\mathrm{X}_{\kappa} \ldots \mathrm{X}_{1}\right)$ exactly specifies whether the edge or vertex has a label in each $\mathcal{E}^{\prime}\left(\mathrm{X}_{j}\right) \Gamma \mathcal{V}^{\prime}\left(\mathrm{X}_{j}\right)$ and if so what this label is. Note however that because vertices may lie on the 
boundary of the supertile they serveГa given vertex may lie in several unrelated skeletons. This is discussed further in the construction of vertex decorations.

We will return to these classes momentarily. The main point here is that the primary information associated with each supertile is to be its label in $\mathcal{R}$. With this specified the role of the supertile in all higher level skeletons is fixed.

\subsection{Vertex-wires $\mathcal{W}$ and wire-keys $\mathcal{U}$}

In the proof of the matching rules $\Gamma$ we will need each supertile's key to arrive at

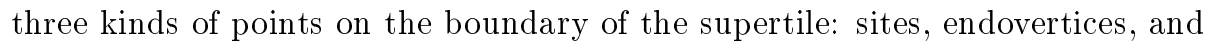
mesovertices. The first two kinds of points lie on the supertile's skeletonTand thus are dealt with.

We require a second Tindependent structure - vertex wires - to send information to the mesovertices of a supertile; more generally we can deliver information to any rational address $\Gamma$ defined momentarily.

Recall that a mesovertex $v \in \mathcal{V}(\mathrm{A}) \Gamma \mathrm{A} \in \mathcal{S}$ lies on boundary of $\mathrm{A}$ and at the end of an edge in $\mathcal{E}\left(\mathrm{A}^{-}\right) \Gamma$ but that for no $n$ does $\sigma^{n}(v)$ lie at the end of any edge in the interior of $\sigma^{n}(\mathrm{~A})$. For each $\mathrm{A} \in \mathcal{S}$ ए let $\mathcal{V}_{m}(\mathrm{~A}) \subset \mathcal{V}(\mathrm{A})$ be the collection of mesovertices of $A \Gamma$ and let $\mathcal{V}_{m}$ be the disjoint union of the $\mathcal{V}_{m}(\mathrm{~A})$ as $\mathrm{A}$ ranges over $S$.

Lemma 2.6 Let $v$ be a vertex of a prototile A. Then $v$ has rational address.

Proof For all $n \in \mathrm{N} \Gamma \sigma^{n}(v)$ is incident to exactly one tile $\Gamma$ congruent to $\mathrm{X}_{n} \in \mathcal{S} \Gamma$ and is coincident with a vertex $v_{n} \in \mathcal{V}\left(\mathrm{X}_{n}\right)$ of this tile. Note that the address of $v$ is thus $\mathrm{A} \bullet \mathrm{X}_{1} \mathrm{X}_{2} \ldots$. Since $\mathcal{V} \Gamma \mathcal{S} \Gamma$ are finite and vertices are hereditary $\Gamma$ this

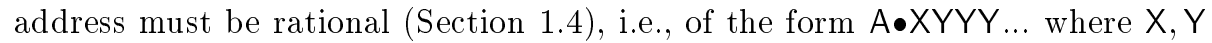
are finite addresses. (In fact $\Gamma$ this is precisely the single point in the construction at which hereditary vertices are invoked)

qed

Let $v \in \mathcal{V}_{m}(\mathrm{~A})$. There are non-negative integers $l(v) \Gamma m(v)$ such that we may suppose $\mathrm{X}=\mathrm{X}_{1} \ldots \mathrm{X}_{l(v)}$ and $\mathrm{Y}=\mathrm{X}_{(l(v)+1)} \ldots \mathrm{X}_{m(v)}$ Tand that for $j, k>l(v) \Gamma$ if $k=j(\bmod (m(v)-l(v))) \Gamma$ then $\sigma^{k}\left(x_{j}\right)=\sigma^{j}\left(x_{k}\right)$ (taking $v$ as the fixed origin) $\Gamma$ and $\left(v_{j}\right)=\left(v_{k}\right) \in \mathcal{V}$. That is $\Gamma$ we require that $v_{k}$ occupies the same position and orientation in $\mathrm{X}_{k}$ as does $v_{j}$ in $\mathrm{X}_{j}$ (figure 4 ).

Let $\mathcal{W}(v)$ be this sequence $\left[\left(\mathrm{A}=\mathrm{X}_{0}\right) \bullet \mathrm{X}_{1}, \ldots, \mathrm{X}_{l(v)}, \mathrm{X}_{(l(v)+1)}, \ldots \mathrm{X}_{m(v)}\right]$. We will take as implicit $\Gamma$ given $\mathrm{X}_{i} \in \mathrm{W}(v) \Gamma$ the values of $l(v)$ and $m(v) \Gamma$ the label 
$v_{i} \in \mathcal{V}\left(\mathrm{X}_{i}\right) \Gamma$ the unique successor $\mathrm{X}_{(i+1)}$ (or $\Gamma$ if $\left.i=m(v) \Gamma \mathrm{X}_{(l+1)}\right)$ and (for $i \neq$ $(l+1))$ the unique predecessor $\mathrm{X}_{(i-1)}$ Tand for $\mathrm{X}_{(l+1)}$ the two predecessors $x_{l}$ and $x_{m}$. To make indexing easier $\Gamma$ for any $n>m \Gamma \mathrm{X}_{n}$ will be taken to mean $\mathrm{X}_{k}$ where $l<k \leq m$ and $n=k(\bmod (m-l))$. Define for each $\mathrm{X}_{i}$ a set of possible preceders - strings drawn from $X Y Y Y$... ending in $X_{i}$ Teither with length $\kappa$ or with length less than $\kappa$ and beginning with $\mathrm{X}_{1}$.

$\mathcal{W}(v)$ is the vertex-wire for the mesovertex $v$. Let $\mathcal{W}$ be the disjoint union over all mesovertices of the $\mathcal{W}(v)$. As usual $\Gamma$ given a $w \in \mathcal{W}$ Гit is implicit for which $v$ that $w$ is an element of $\mathcal{W}(v)$.

We next define for any tiling in $(T \Gamma \sigma \Gamma \mathcal{S})$ a map $\lambda_{\mathcal{U}}: V \rightarrow(\mathcal{W} \times \mathcal{R}) \cup\{$ null $\}$ where $V$ is the set of all epivertices of the supertiles in the tiling:

Recall Lemma 1.6.

In particularTany epivertex $v$ of a supertile $\sigma^{j}(\mathrm{~B})$ in a tiling is either on the exterior of any supertile to which it is incident or either incident some lower level edge in the interior of the supertile $\Gamma$ or is coincident to a mesovertex $w$ of some higher level supertile $\sigma^{n}(\mathrm{~A}) \Gamma$ with $\lambda_{\mathcal{R}}\left(\sigma^{n}(\mathrm{~A})\right)=\mathrm{R} \in \mathcal{R}$. In the first and second cases $\Gamma$ take $\lambda_{\mathcal{U}}(v)=$ null . Otherwise take $\lambda_{\mathcal{U}}(v)=\left(\mathrm{X}_{(n-j)}, \mathrm{R}\right)$ where $\mathrm{X}_{(n-j)}$ is the $(n-j)$ th digit of the vertex wire $\mathcal{W}(w)$.

The possible images of epivertices are wire-keys; let $\mathcal{U}(v) \subset(\mathcal{W} \times \mathcal{R}) \Gamma$ be the set of possible wire keys of a particular epivertex $v \in \mathrm{A} \in \mathcal{S} \Gamma$ and let $\mathcal{U}$ be the disjoint union of the $\mathcal{U}(v)$.

\subsection{Supertile packets $\mathcal{Q}$, edge- and vertex-packets $\mathcal{P}$}

We now summarize the classes of labels associated with each primary structure. Packets will be certain bundles of labels associated with various structures. Edge packets and vertex packets will $\Gamma$ in essence $\Gamma$ be the markings for our new tiles.

A given supertile $\sigma^{n}(\mathrm{~A}) \Gamma \mathrm{A} \in \mathcal{S}$ will carry a key $\mathrm{R} \in \mathcal{R}(\mathrm{A})$ of its own and wire keys $\mathrm{U} \in \mathcal{U}(v)$ for each of epivertex $v$ in $\mathcal{V}(\mathrm{A})$. For each $\mathrm{A} \in \mathcal{S}$ एlet $\mathcal{Q}(\mathrm{A})$ be the supertile packets - sets of labels for supertiles $\sigma^{n}(\mathrm{~A})$ : that is sets of the form $\left[\mathrm{R}, \mathrm{U}_{1}, \mathrm{U}_{2}, \ldots\right]$ such that $\mathrm{R} \in \mathcal{R}(\mathrm{A})$ and there is exactly one $\mathrm{U}_{i} \in \mathcal{U}(v)$ for each epivertex $v$ in $\mathcal{V}(\mathrm{A})$ and each $\mathrm{U}_{i}$ lies in some $\mathcal{U}(v)$ for some epivertex $v$ in $\mathcal{V}(\mathrm{A})$. Note these sets of labels are finite and there are finitely many such sets.

Take $\mathcal{Q}$ to be the disjoint union of the $\mathcal{Q}(\mathrm{A})$ over $\mathcal{S}$. We also include an extra null label in $\mathcal{Q}$. 
Given a tiling $\cup \mathrm{B}_{i} \mathrm{~A}_{i}$ in $(T \Gamma \sigma \Gamma \mathcal{S}) \Gamma$ define a map $\lambda_{\mathcal{Q}}:\left\{\sigma^{n}(\mathrm{~A}) \mid n \in \mathrm{N}, \mathrm{A} \in\right.$ $\left.\mathcal{S}, \sigma^{n}(\mathrm{~A}) \subset \cup \mathrm{B}_{i} \mathrm{~A}_{i}\right\} \rightarrow \mathcal{Q}$ with $\Gamma$ for supertile $\sigma^{n}(\mathrm{~A})$ with epivertices $v_{i} \Gamma \lambda_{Q} Q\left(\sigma^{n}(\mathrm{~A})\right)=$ $\left[\lambda_{\mathcal{R}}, \lambda_{\mathcal{U}}\left(v_{1} \in \sigma^{n}(\mathrm{~A})\right), \ldots\right]$.

Lemma 2.7 Let $\left[\mathrm{R}, \mathrm{U}_{1}, \mathrm{U}_{2}, \ldots\right]$ be the image under $\lambda_{\mathcal{Q}}$ of some supertile in a tiling with connected hierarchy. For each non-null $\mathrm{U}_{i}=\left[\mathrm{Y}_{n_{i}}, \mathrm{R}_{i}\right]$ with $\mathrm{Y}_{n_{i}} \in$ $\mathrm{W}(v)$, note $\mathrm{R}$ is either a possible preceder of $\mathrm{Y}_{n_{i}}$ or terminates in a possible preceder of length $k<\kappa$ of $\mathrm{Y}_{n_{i}}$. In the latter case, we further note the last $(\kappa-k)$ digits of $\mathrm{R}_{i}$ are the first $(\kappa-k)$ digits of $\mathrm{R}$.

Proof This follows immediately from the definitions.

qed

The defining label for each edge of every tile is a label in $\mathcal{E}^{\prime \prime}$. Thus a given edge of a tile will convey a edge packet $\left[e, Q_{1} \Gamma Q_{2} \Gamma \ldots Q_{\kappa}\right] \in\left(\mathcal{E}^{\prime \prime} \times \mathcal{Q}^{\kappa}\right) \cup\{$ null $\}$ of labels $\Gamma$ consisting of its own label $e \in \mathcal{E}^{\prime \prime}\left(\mathrm{X}_{\kappa} \ldots \mathrm{X}_{1}\right) \in \mathcal{E}^{\prime \prime} \Gamma$ and a finite collection of labels in $\mathcal{Q}$. The first element $\Gamma e \Gamma$ will be the header of the packet; the rest will be the trailer.

Given a tiling in $(T \Gamma \sigma \Gamma \mathcal{S}) \Gamma$ let $E$ be the set of all points of edges of tiles in the tiling and define $\lambda_{\mathcal{P}}: \mathcal{E} \rightarrow\left(\mathcal{E}^{\prime \prime} \times \mathcal{Q}^{\kappa}\right) \cup\{$ null $\}$ :

Recall each point $f \in E$ of each edge either lies on an infinite fault line or lies in some $\sigma^{n}(e) \Gamma e \in \mathcal{E}^{\prime \prime}\left(\mathrm{X}_{\kappa} \ldots \mathrm{X}_{1}\right) \Gamma$ and thus lies within up to $\kappa$ skeletons $\sigma^{(n+k-1)}\left(E_{\mathrm{X}_{k}}\right) \Gamma \kappa \in K \subset\{1, \ldots, \kappa\} \Gamma$ with $1 \in K$ (This is given by $\left.\lambda_{\mathcal{E}^{\prime \prime}}(f)\right)$. In the first case let $\lambda_{\mathcal{P}}(f)=$ null; in the second let $\lambda_{\mathcal{P}}(f)=\left[e, Q_{1}, Q_{2}, \ldots, Q_{\kappa}\right] \Gamma$ where $\mathrm{Q}_{i}=\lambda_{\mathcal{Q}}\left(\sigma^{(n+k-1)}\left(\mathrm{X}_{k}\right)\right)$ for $i \in K$ and $\mathrm{Q}_{i}=$ null otherwise.

The image of $E$ under $\lambda_{\mathcal{P}}$ will be edge packets $\mathcal{P}$.

Thus given edge will carry a packet in $\mathcal{P}$ Twhich amounts to a label in $\mathcal{E}^{\prime \prime}$ for the lowest level skeleton to which the edge belongs and $\kappa$ keys for supertiles of the next $\kappa$ levels in the hierarchyГand any wire keys these supertiles are in turn transmitting.

The following amounts to a long lemma with trivial proof following immediately from the construction:

Any packet in $\left[e, \mathrm{Q}_{1} \Gamma \mathrm{Q}_{2} \Gamma \ldots \mathrm{Q}_{\kappa}\right] \in \mathcal{P} \Gamma e \in \mathcal{E}^{\prime \prime}\left(\mathrm{X}_{\kappa} \ldots \mathrm{X}_{1}\right)$ satisfies:

Let each non-null $\mathrm{Q}_{i}=\left[\mathrm{R}_{i}, \mathrm{U}_{i 1}, \mathrm{U}_{i 2}, \ldots\right]$. We must have $R_{1}=\mathrm{X}_{\kappa} \ldots \mathrm{X}_{1}$. 
For each pair of non-null $\mathrm{Q}_{i}, Q_{j} \Gamma \mathrm{R}_{j}=\mathrm{X}_{(\kappa, j)} \ldots \mathrm{X}_{(1, j)} \Gamma \mathrm{R}_{i}=\mathrm{X}_{(\kappa, i)} \ldots \mathrm{X}_{(1, i)}$ with $1 \leq j<i \leq \kappa$ in a packet for label $e \in \mathcal{E}^{\prime \prime} \Gamma$ we have $\mathbf{X}_{(k-i, i)}=\mathbf{X}_{(k-j, j)}$ for $i<k \leq j+\kappa$ and $e \in \mathcal{E}^{\prime \prime}\left(R_{1}\right)$. That is $\Gamma$ we can define $\Gamma$ for well formed edge packets $\Gamma$ a longer address $\mathrm{X}_{j} \ldots \mathrm{X}_{\kappa} \ldots \mathrm{X}_{1}$ of some length $j \Gamma \kappa \leq j \leq(2 \kappa-1)$ such that for any $1 \leq k \leq j \Gamma 1 \leq i \leq k \Gamma \mathrm{X}_{k}=\mathrm{X}_{(i, k-i)}$; the $\mathrm{R}_{i}$ are in effect snippets of this longer address. (The length $j$ depends on which $\mathrm{R}_{j}$ and $\mathrm{Q}_{j}$ are not vacant $\Gamma$ that is $\Gamma$ on which higher level skeletons $e$ serves.)

Given the longer address $\mathrm{X}_{j} \ldots \mathrm{X}_{\kappa} \ldots \mathrm{X}_{1}$ Tit is clear by examining $\sigma^{j}\left(\mathrm{X}_{j}\right)$ exactly how the vertices of any $\mathrm{X}_{k} \Gamma 1 \leq k \leq \kappa$ coincide with vertices of any other $\mathrm{X}_{l} \Gamma$ $1 \leq l \leq \kappa$. We note the $\mathrm{U}_{i j}$ do not conflict at these vertices:

In particular $\Gamma$ suppose $v_{k}$ is an epivertex of $\mathrm{X}_{k} \Gamma$ with corresponding $\mathrm{U}_{k i} \Gamma$ incident to an epivertex $v_{l}$ of $\mathrm{X}_{l} \Gamma$ with corresponding $\mathrm{U}_{l i}$. Then $\mathrm{U}_{k i}$ is null in $\mathcal{U}\left(v_{k}\right)$ if and only if $\mathrm{U}_{l i}$ is null in $\mathcal{U}\left(v_{l}\right)$; if neither is null $\Gamma$ let $\mathrm{U}_{k i}=\left(\mathrm{Y}_{n_{k i}} \in\right.$ $\left.\mathrm{W}\left(v_{k i}\right), \mathrm{R}_{k i}\right)$ Tand $\mathrm{U}_{l i}=\left(\mathrm{Y}_{n_{l i}} \in \mathrm{W}\left(v_{l i}\right), \mathrm{R}_{l i}\right)$. Then we must have $v_{k i}=v_{l i} \in \mathcal{V}_{m} \Gamma$ $n_{k i}+l=n_{l i}+k\left(\bmod \left(m\left(v_{k i}\right)-l\left(v_{k i}\right)\right.\right.$ and $\mathrm{R}_{k i}=\mathrm{R}_{l i}$.

Suppose $v_{k}$ is an epivertex of $\mathrm{X}_{k}$ Twith corresponding $\mathrm{U}_{k i}$ Tincident to some mesovertex $v$ of $v_{l}$ of $\mathrm{X}_{l}$; then $\mathrm{U}_{k i}$ must not be null. Let $\mathrm{U}_{k i}=\left(\mathrm{Y}_{n_{k i}} \in\right.$ $\left.\mathrm{W}\left(v_{k i}\right), \mathrm{R}_{k i}\right)$. We require $\mathrm{R}_{l}=\mathrm{R}_{k i} \Gamma v=v_{k i} \Gamma$ and $\mathrm{Y}_{n_{k i}} \Gamma$ the $\left(n_{k i}\right)$ th digit of $W\left(v_{k i}\right)$.

We are not particularly concerned with endovertices incident to $v_{k}$ at this moment.

Note that for supertiles $\sigma^{n-1}(\mathrm{~B}) \subset \sigma^{n}(\mathrm{~A}) \Gamma \mathrm{B} \in \mathrm{A}^{+} \Gamma$ the above conditions on the $Q_{i}$ satisfied by a well-formed edge packet will hold $\Gamma$ taking $Q_{1}=\lambda_{\mathcal{Q}}\left(\sigma^{n-1}(B)\right)$ and $\mathrm{Q}_{2}=\lambda_{\mathcal{Q}}\left(\sigma^{n}(\mathrm{~A})\right)$.

If $\kappa=1 \Gamma$ we can compare the packet $\mathrm{Q}_{1}=\left[\mathrm{A}, \mathrm{U}_{11}, \mathrm{U}_{12}, \ldots\right]$ conveyed by a daughter tile to the packet $Q_{2}\left[B, U_{21}, U_{22}, \ldots\right]$ of its parent. We will say $Q_{1}$ and $\mathrm{Q}_{2}$ are paired if $\mathrm{A} \in \mathrm{B}^{+}$and the conditions on the $U_{i j}$ must be satisfied as above.

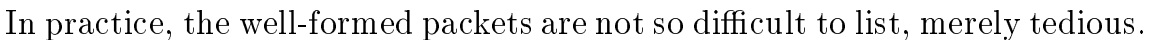
The restrictions simply ensure the packets are not so malformed as to never arise in an actual tiling. Let $\mathcal{P}\left(\mathcal{E}^{\prime \prime}\right)$ be the collection of these well-formed edge packets.

We similarly define vertex packets $\left[v, Q_{1} \Gamma Q_{2} \Gamma \ldots Q_{\kappa}\right]$ of labels $\Gamma$ consisting of a vertex label $v \in \mathcal{V}^{\prime \prime}\left(\mathrm{X}_{\kappa} \ldots \mathrm{X}_{1}\right) \in \mathcal{V}^{\prime \prime} \Gamma$ and a finite collection of labels in $\mathcal{Q}$. We only define such packets for vertices $\mathcal{V}^{\prime \prime}\left(\mathrm{X}_{\kappa} \ldots \mathrm{X}_{1}\right)$ incident to the skeleton of $\mathrm{X}_{1}$.

As above $\Gamma$ let $v$ be the header of the packet $\left[v, Q_{1} \Gamma Q_{2} \Gamma \ldots Q_{\kappa}\right]$ and the $Q_{i}$ the trailer of the packet. Note that our description of well-formed edge-packet made no real use that the first item in such a packet is a edge label in $\mathcal{E}^{\prime \prime}$; we 
thus make the same restrictions in defining well-formed vertex packets and let $\mathcal{P}\left(\mathcal{V}^{\prime \prime}\right)$ be the collection of all well formed vertex packets. For each element of $\mathcal{P}\left(\mathcal{V}^{\prime \prime}\right)$ we can also define a longer address as we did for the $\mathcal{P}\left(\mathcal{E}^{\prime \prime}\right)$.

A given edge will carry a packet in $\mathcal{P} \Gamma$ which amounts to a label in $\mathcal{E}^{\prime \prime}$ for the lowest level skeleton to which the edge belongs and $\kappa$ keys for supertiles of the next $\kappa$ levels in the hierarchyГand any wire keys these supertiles are in turn transmitting.

Finally $\Gamma$ we define $\Gamma$ given a tiling in $(T \Gamma \sigma \Gamma \mathcal{S}) \Gamma$ a map $\lambda_{\mathcal{F}}$ mapping the points of each 1 -facet of each edge of each tile of a tiling into $([0,1] \times \mathcal{F}) \cup\{$ null $\} \Gamma$ as follows:

Every edge of every tile $f$ in the tiling either lies on a infinite fault-line or lies in a unique $\sigma^{n}(e) \Gamma e \in \mathcal{E}^{\prime \prime}$. In the first case $\Gamma$ take $\lambda_{\mathcal{F}}(h)=$ null.

In the second case every 1-facet $h$ of $f$ may or may not lie within a 1-facet of $\sigma^{n}(e)$. If it does not $\Gamma$ take $\lambda_{\mathcal{F}}(h)=$ null (this only occurs when $\mathrm{n}>2$ ). Otherwise $\Gamma$ $h$ lies within a 1-facet $\operatorname{sigma}^{n}\left(h^{\prime}\right)$ of $\sigma^{n}(e) \Gamma h^{\prime} \in \mathcal{F}(e)$ (Section 2.2.3). Recall $h^{\prime}$ has an assigned orientation in $e$ (an arrow pointing from one end of $h$ to the other); let $h$ inherit this orientation from $\sigma(e)$.

Thus $h$ has a "high end" and a "low end" $\Gamma$ lying towards the head and tail respectively of the arrow on $\sigma\left(h^{\prime}\right)$. Let $\lambda_{\mathcal{F}}$ linearly map $h$ to $[0,1] \times\left\{h^{\prime}\right\}$ with the high end of $h$ mapped to $1 \Gamma$ the low to 0 .

This last map will serve to orient our labeling in the tiling; recall that the set of 1 - facets of the tiles in the tiling is connected. 


\section{Creating tiles and markings}

We will define new prototiles $\mathcal{T}^{\prime}$ and matching rules $\mathcal{M}$ for piecing them together.

We will then explicitly define a labeling of $(\mathcal{T} \Gamma \sigma \Gamma \mathcal{S})$. In effect $\Gamma$ on either side of each edge in a tiling we will mark with the appropriate edge packet; the middle of each tile will be marked with an appropriate supertile packet.

We will then show this new matching rule tiling $\left(\mathcal{T}^{\prime} \Gamma \mathcal{M}\right)$ reconstructs the labeling.

We will describe three flavors of proto-tile and then markings derived from the various sets of labels. Here we use the finiteness of $\mathcal{V}$ to ensure that we can find a finite collection of standard tiles employing a finite collection of matching rules.

As a point of interest $\Gamma$ we could merely take for $\mathcal{T}^{\prime}$ the labelings of our original prototiles $\Gamma$ but this technique is as well defined and results in a huge reduction in the number of tiles required.

\subsection{Creating new unmarked tiles}

Lemma 3.1 Given a substitution tiling $(T, \sigma, \mathcal{S})$, there exist $\varepsilon, \delta>0$ such that every point in any prototile of $\mathcal{T}$ that is less than $\varepsilon$ from more than one 1-facet of a prototile is less than $\delta$ from a vertex of the prototile; and such that no point in a tile is within $2 \delta$ of more than one vertex of the tile.

Note that $\mathrm{n}=2$ The 1 -facets of the prototile are the prototile's edges. When $\mathrm{n}>2$ Tnote that the lemma implies that if a point is within $\varepsilon$ of more than one edge of the prototile $\Gamma$ the point is within $\varepsilon$ of a 1-facet of the prototile (recall the the $k$-facets of the prototile all lie within $k$-planes in $\mathrm{E}^{\mathrm{n}}$.

Proof Because the vertices are finite and hence discrete and the edges are confined to $(n-1)$-planes $\Gamma$ for each vertex $v$ of each prototile $A \in \mathcal{T} \Gamma$ there is some $\delta_{v}$ such that every other vertex of A $\Gamma$ and every edge A not meeting $v$ lies outside an open n-ball of radius $\delta_{v}$. Take $2 \delta$ to be the minimum of the $\delta_{v}$ as $v$ ranges over all the vertices of all the prototiles (Since the set of these vertices is finite $\Gamma$ this minimum is attained).

With $\delta$ fixed $\Gamma$ if $\mathrm{n}>2 \Gamma$ for each pair of 1 -facets $e, f$ in a prototile $A \in \mathcal{T} \Gamma$ there is an $\varepsilon_{e, f}$ such that if a point $x \in \mathrm{A}$ lies less than $\varepsilon_{e, f}$ of both $e$ and $f \Gamma$ then $x$ lies within $\delta$ of some vertex of A. Take $\varepsilon$ to be the minimum of the $\varepsilon_{e, f}$ as $e$ and $f$ range over all pairs of 1 -facets in each prototile.

$$
\text { qed }
$$


We define a set $\lambda^{-1}(\partial)$ of points in each tiling as follows (this set will both define the boundaries of our new tiles $\mathcal{T}^{\prime}$ and begin to define our labeling $\lambda$ ):

First $\Gamma$ any point in any tile in any supertile in any tiling that lies on the boundary of the tile $\Gamma$ exactly $\delta$ from a vertex of a tile or exactly $\varepsilon$ from one edge or equidistant to two or more edges of the tile $\Gamma$ such that this distance is less than $\varepsilon$ and no edge is closer and no closer than $\delta$ to any vertex of the tile will be labeled " $\partial$ ". This will mark the boundaries of our new tiles.

Note that the closures of the components of any tiling less the points marked $\partial$ fall into a finite collection of congruence classes: we partition these classes into unmarked vertex hulls $\Gamma$ edge tiles $\Gamma$ and small tiles $\Gamma$ depending on if they were originally within $\delta$ of a vertex $\Gamma \varepsilon$ of an edge but further than $\delta$ from a vertex $\Gamma$ or further than $\varepsilon$ from an edge and $\delta$ from a vertex.

Note of course that the unmarked vertex hulls are exactly that-congruent to vertex hulls in $\mathcal{V}^{\prime \prime}$ with the markings wiped off.

We will define vertex-tiles as the union of such hulls shortly; vertex-tiles will be marked n-balls (disks) or sectors of n-balls.

Because of problems with vertices and edges of level less than zero $\Gamma$ we will ultimately coalesce small tiles into big tiles $\Gamma$ derived from supertiles of level $\kappa$.

In figure 14 the triangle tiling of figure 7 has been carved up into small tiles $\Gamma$ edge tiles and vertex hulls. The vertex hulls have not yet been assembled into vertex tiles. None of these tiles have yet been marked.

The out-side of an edge-tile is the image of the boundary of the original tile from whence it was derived; the in-side of an edge-tile are the points on the boundary of the edge-tile that are $\varepsilon$ from the image of the boundary of the original tile. The ends of an edge-tile are the remaining points on the boundary of the tile. The edge tiles are marked by $\partial$ on their boundaries and labels in $\mathcal{P}\left(\mathcal{E}^{\prime \prime}\right) \cup\{$ null $\}$ in their interior $\Gamma$ encoded by any convenient scheme $\Gamma$ and by $[0,1] \times \mathcal{F} \cup\{$ null $\}$ on the one-dimensional facets of edges of the tile that allow us to identify the orientation of the tile (note each 1-facet of the tile itself may have several labelings in $[0,1] \times \mathcal{F})$. We call these images of $[0,1] \times\{h\} \Gamma h \in \mathcal{F}$ a sided arrows (the sided refers to the arrow being associated with a particular "side" of the tile- a certain 1-facet of a certain edge). The head of the sided arrow lies at the image of $1 \times\{h\} \Gamma$ the tail at the image of $1 \times\{h\}$. 


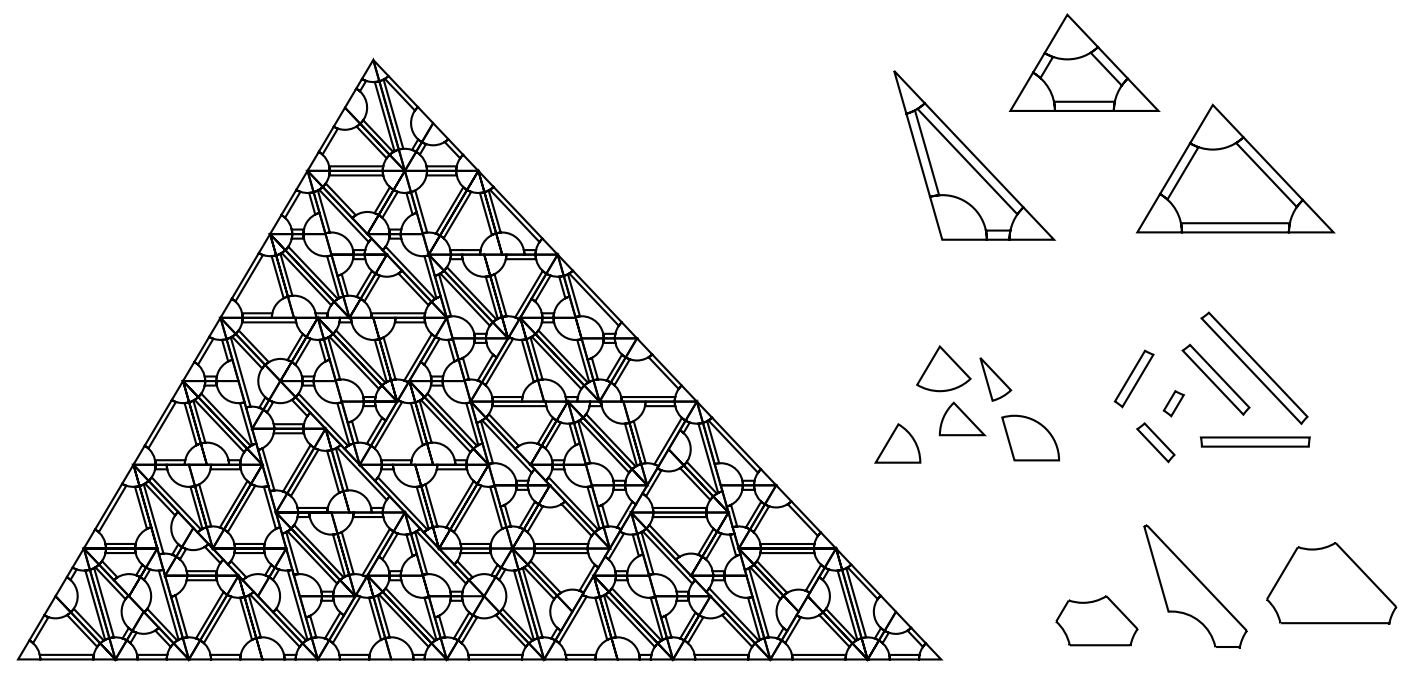

Figure 14: Small tiles $\Gamma$ edge tiles and vertex hulls

\section{$3.2 \quad$ Vertex tiles and vertex markings}

The markings for the vertex tiles are somewhat more complicated since a single tile may carry several markings. An edge has a specific level $\Gamma$ the level of the supertile it bounds. A vertex might be the endpoint of many different levels of edge and so has no specific level of its own. Instead labels in $\mathcal{V}^{\prime \prime}$ lie at specific levels and are the basis for our markings. Every vertex has finite valence and there are a finite number of vertex configurations; thus there is a bound on the number of markings required. We allow all combinations with the correct valences $\Gamma$ satisfying certain compatibility requirements given below. The compatibility rules ensure a great degree of redundancy and in practice $\Gamma$ one can sort out the actual combinations of marking that are required.

Recall from Section 2.2.3 that the labels $\mathcal{V}^{\prime \prime}$ each have some defined hull Tan $\mathrm{n}$-ball or a portion of a $\mathrm{n}$-ball. The labels in $\mathcal{P}\left(\mathcal{V}^{\prime \prime}\right)$ and the hulls of their headers will form the basic vertex markings. Unions of these markings will form vertex tiles.

We will also view a vertex tile as the co-dimension 1 projection of a stack of these markings from an $(n+1)$ th dimension. The relative height of two markings corresponds to their relative height in the hierarchical structure on the tiling. Such stacks have bounded height. If we literally allow the stacking of tilesГand 
take the vertex markings as tilesTthe number of tiles needed drops dramatically. The compatibility rules $\Gamma$ defined shortly $\Gamma$ are in effect vertical matching rules describing permitted stacks of markings. We will instead project these stacks down into disks or sectors $\Gamma$ marked with many markings ranked by height $\Gamma$ and take these as our vertex tiles.

A label $v \in \mathcal{V}^{\prime \prime}$ belongs to one of several classes. Let $v \in \mathcal{V}^{\prime \prime}\left(\mathrm{X}_{\kappa} \ldots \mathrm{A}\right)$.

i) either $v$ is internal and lies in the interior of $\mathrm{A}$ or is external

and lies on the boundary of $A$.

ii) $v$ arose as any or all of:

a) the endpoint of edges of relative level 1 . Some of these will be among the sites serving $A$.

b) a site serving a lower level tile $\Gamma$ an element of $A^{+}$.

c) the endpoint of some edge $\sigma^{(k-\kappa)}(e) \Gamma e \in \mathcal{E}^{\prime}\left(\mathrm{X}_{k}\right)$;

in particularTthis may be a point where the skeleton of $\sigma^{k}\left(\mathrm{X}_{k}\right) \Gamma 1<k \leq \kappa$ departs $\mathcal{E}(\mathrm{A})$ into some element of $\mathrm{A}^{+}$.

d) if $v$ is external $\Gamma$ a site for some $\sigma^{k}\left(\mathrm{X}_{k}\right) \Gamma 1 \leq k \leq \kappa$

e) an isolated vertex on the boundary of $A$.

Note that all of the internal vertices and some of the external vertices of $\sigma^{n}(\mathrm{~A})$ labeled in $\mathcal{V}^{\prime \prime}(\mathrm{A})$ are terminals of the $\sigma^{(n-1)}\left(\mathrm{A}^{+}\right)$.

A vertex marking is a label $P \in \mathcal{P}\left(\mathcal{V}^{\prime \prime}\right) \Gamma$ with the hull of its header Toriented so that the position of all edges incident to the header can be determined. The hull of a vertex marking is just the hull of its header. The dark edges of a vertex marking are the edges darkly marked in the hull of the marking. Recall that the hull of a vertex in $\mathcal{V}^{\prime \prime}$ is marked with the positions Tlabels in $\mathcal{E}^{\prime}$ and relative levels of its dark edges $\Gamma$ and the 1 -facets of each edge have been labeled in $\mathcal{F}$ and oriented with arrows.

If the hull includes no dark edges of relative level 1 the trailer is null. If the header of $P$ includes a dark edge of relative level 1 we regard this edge as being marked in $\mathcal{P}\left(\mathcal{E}^{\prime \prime}\right)$ with the header of this marking corresponding to the label in $\mathcal{E}^{\prime}$ of this edge; we further take the trailer of this marking and the trailer of $P$ to be the same.

Similarly the light edges of a vertex marking are the lightly marked edges in its hull; the flat sides of a vertex marking are the flat sides of the hull.

One more vertex marking is possible $\mathrm{Tan}$ overpass; this is simply a join for extending edges indefinitely. Such an overpass is a sector of an $n$-ball; each flat side must consist of a single $k$-plane $\Gamma 0<k<\mathrm{n}$. Such an overpass is to be only the highest vertex marking in a stack of markings.

If the sector has two flat sides of dimensions $j, k$ These must share at least a 
common $(\mathrm{n}-j-k)$-plane of intersection in the boundary of the sector (hence when $\mathrm{n}=2$ Tan overpass has only one flat side). This line must be marked with the orientations and labels in $\mathcal{F}$ of the 1 -facets of the edges propagated by the overpass.

Along this line an overpass propagates the boundary between higher level edges in the tiling $\Gamma$ and also carries the orientations and labels in $\mathcal{F}$ of the 1facets of these higher level edges. (Such a structure is necessary anywayГand helps us avoid defining tiles for every dimension from zero up through $n$ Tinstead of just for dimensions $0 \Gamma(n-1)$ and $n)$.

\subsection{Compatibility rules}

We specify how we allow the vertex markings to stack:

First $\Gamma$ the disk markings are given an order in height. The higher markings correspond to higher-level vertex hulls; they thus will be at least a large as portion of a n-ball than the lower markings. The highest marking must either be an overpass or a complete $n$-ball; the lowest markings must be unmarked sectors arising from hulls in $\mathcal{V}^{\prime \prime}$ meeting no edges. In between $\Gamma$ we require that each flat side of a hull must lie exactly beneath a flat side or a edge of the next marking up; that edges and the flat sides of a hull must lie above the flat side of the hull of the next marking down; and that every light edge must be above some dark edge with the same marking in $\mathcal{E}^{\prime \prime}$.

Suppose two vertex markings $P_{1}, P_{2} \in \mathcal{P}\left(\mathcal{V}^{\prime \prime}\right)$ contain dark edges that coincide when the stack is projected to a single tile; let the first dark edge have relative level $j$ to $P \Gamma$ the second relative level $k$ to $Q$. We require the edges' sided arrows must be oriented the same way and have the same labels in $\mathcal{F}$ and the edges' labels in $\mathcal{E}^{\prime}$ must be the same; $|j-k|$ must be less than $\kappa$. Furthermore $\Gamma$ if both $P_{1}, P_{2}$ have non-null trailers $\Gamma$ say $\left[\ldots, \mathrm{Q}_{11}, \mathrm{Q}_{12}, \ldots, \mathrm{Q}_{1 \kappa}\right] \Gamma\left[\ldots, \mathrm{Q}_{21}, \mathrm{Q}_{22}, \ldots, \mathrm{Q}_{2 \kappa}\right]$ we require that $\mathrm{Q}_{2(i+j)}=\mathrm{Q}_{1(i+k)}$ for $0 \leq i \leq(\kappa-|j-k|-1)$.

Finally $\Gamma$ consider the vertex marking $P \in \mathcal{P}\left(\mathcal{V}^{\prime \prime}\right) \Gamma$ where the header $v$ of $P$ arose as a site serving a tile $B \subset \sigma(A) \Gamma v \in \mathcal{V}^{\prime \prime}\left(\mathrm{X}_{k} \ldots \mathrm{AB}\right)$. If $\kappa=1 \Gamma$ the trailers of the packets must be paired $\Gamma$ as defined in Section 2.4. Otherwise $\Gamma$ let $P=\left[v, \mathrm{Q}_{1}, \ldots \mathrm{Q}_{\kappa}\right]$. The hull of the marking must be a sector. Immediately above this sector must be a marking labeled $P^{\prime}$ with header $w \in \mathcal{V}^{\prime \prime}\left(\mathrm{X}_{(\kappa+1)}, \mathrm{X}_{\kappa}, \ldots, \mathrm{A}\right)$ and $P=\left[w, Q_{2}, \ldots, Q_{\kappa}, Q_{(\kappa+1)}\right]$. By the definitions of $\mathcal{E}^{\prime \prime} \Gamma \mathcal{V}^{\prime \prime} \Gamma$ these two markings have at least one edge that coincide when the stack is projected to a tile; thus we are assured that $w$ and $v$ are appropriately matched (as they are endpoints of these edges $\Gamma$ and the orientations and labels in $\mathcal{F}$ of the 1-facets match). The point is that a vertex tile serving as a site for a supertile must connect what appears to be the appropriate edge for the lower level skeleton to what appears 
to be the appropriate edge for the higher level skeleton $\Gamma$ that the orientations and labels in $\mathcal{F}$ of the 1 -facets of these edges are correct $\Gamma$ and that the packets on these skeletons are appropriate as the packets of child and parent supertiles.

The choice of $Q_{(\kappa+1)}$ is the engine of aperiodicity.

We now define vertex tiles: given a stack of vertex hulls satisfying the compatibility rules $\Gamma$ project the stack to a $\mathrm{n}$ ball or sector. Mark all the images of the edges of hulls with $\partial$. In an $\varepsilon$ neighborhood of these marks by $\partial \Gamma$ mark the edge packet of the lowest dark edge above the projection.

\subsection{Small tiles}

We defined small tiles $\Gamma a b o v e \Gamma a s$ simply prototiles with vertex and edge tiles cut away. We label these with packets in $\mathcal{Q} \Gamma$ for small tiles are really just marred little supertiles. If a small tile's packet contains any non-null $U \in \mathcal{U} \Gamma U=[X, R] \Gamma$ then the tile has a terminator $\Gamma$ the label $W$ in $\mathcal{W}$ corresponding to the vertex wire determined by $\mathrm{U} \Gamma$ the label $\mathrm{R}$ carried by $\mathrm{U}$ and the position and orientation of $\mathrm{X}$ with respect to $\mathrm{X}_{1} \in \mathcal{W}$. The label $v$ in $\mathcal{V}$ such that $\mathrm{W} \in \mathcal{W}(v)$ is implied. The terminators are the whole motivation for vertex wires.

Recall that small neighborhoods about vertices have been deleted from the original prototiles. Thus Tif $\mathbf{X}=\mathrm{X}_{i} \in \mathrm{W}(v) \Gamma$ then the terminal is marked on the boundary of a neighborhood of $v_{i}$ in $\mathrm{X}$; the terminal is an orientation fixing the position of $v$ and a marking with the supertile packet $\mathrm{R}$ and vertex label $v$.

For any $\mathrm{A} \in \mathcal{T}$ Tthere is some $k$ such that for all $n$ Tif an edge $e$ in $\sigma^{n}(\mathrm{~A})$ meets a vertex $\sigma^{n}(v) \Gamma v \in \mathcal{V}(A) \Gamma$ then there is an edge $\sigma^{(k-n)}(e)$ meeting $\sigma^{k}(v) \in \sigma^{k}(\mathrm{~A})$; that is $\Gamma$ after some number $k$ of subdivisions $\Gamma$ no further edges are incident to the vertices of A. Lightly mark $\sigma^{-k}(e) \subset \mathrm{A} \Gamma$ for all $e \subset \sigma^{k}(\mathrm{~A})$ such that $e$ is incident to a vertex $\sigma^{k}(v)$. Given $\mathrm{Q} \in \mathcal{Q}(\mathrm{A}) \Gamma$ the edge packets are fixed for each

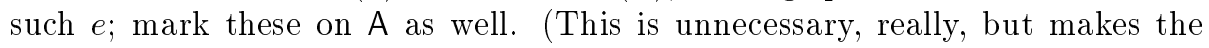
matching rules more straightforward).

\subsection{Matching Rules $\mathcal{M}$ and tiles $\mathcal{T}^{\prime}$}

We define $\mathcal{T}^{\prime}$ as the marked edge tiles $\Gamma$ vertex tiles and small tiles.

The terminal matching rule: a terminator marked $\mathrm{R}=\mathrm{X}_{k} \ldots \mathrm{X}_{2} \mathrm{X}_{1}$ and $v$ must be incident to a vertex tile with a terminal vertex marking $v^{\prime \prime} \in \mathcal{V}\left(\mathrm{X}_{2}\right)$ such that $\left(v \in \sigma\left(\mathrm{X}_{2}\right)\right)=\sigma\left(v^{\prime \prime} \in \mathrm{X}_{2}\right)$ and that $v^{\prime \prime}$ is aligned correctly with the orientation on the boundary of the terminator. 
We give the remaining rules. Essentially these are just that edges and vertices should fit together properly. The rules are edge to edge $\Gamma$ and once all our labels our defined it really is just a matter of matching markings. Of course Tadditional rules have already been encoded in the compatibility rules for the vertex-tile markings $\Gamma$ the definition of well formed packets in $\mathcal{P} \Gamma$ the redefinition of big tiles $\Gamma$ the listing of the vertex hulls $\Gamma$ and the definition of the vertex wires.

The edge matching rule: The end of an edge tile marked $\left[e, Q_{1}, \ldots, Q_{k}\right]$ must meet a vertex tile such that with a vertex marking $\left[v, Q_{1}, \ldots Q_{k}\right]$ with e marked as a dark edge of relative level 1 ; the orientations and labels in $\mathcal{F}$ of the sided arrows must match as well. The in-side of an edge tile must meet a small tile (or big tile, defined shortly). Any point on the interior of the out-side of an edge tile must meet the flat side of a vertex tile (an overpass) with the same marking and orientation, but opposite sidedness, or a vertex tile marked with an overpass marked with the same orientation and marking and opposite sidedness.

The tiling rule: The tiles must cover the plane and have disjoint interiors.

This last rule forces vertex tiles to fit in the disk-shaped holes in the small tiles. Edges to fit along the sides of the small tiles. ${ }^{8}$

We will give one final rule momentarily Tafter we formally define our labeling.

\subsection{The labeling $\lambda$}

Recall that we have defined:

$\lambda_{\mathcal{Q}}:\left\{\sigma^{n}(\mathrm{~A}) \mid n \in \mathrm{N}, \mathrm{A} \in \mathcal{S}, \sigma^{n}(\mathrm{~A}) \subset \cup \mathrm{B}_{i} \mathrm{~A}_{i}\right\} \rightarrow \mathcal{Q}$.

$\lambda_{\mathcal{P}}: E \rightarrow \mathcal{P}$ where $E$ is the set of all points of edges of tiles in a tiling in $(T \Gamma \sigma \Gamma \mathcal{S})$.

$\lambda_{\mathcal{F}}: \mathcal{F} \rightarrow([0,1] \times \mathcal{F}) \cup\{$ null $\}$ Twhere $F$ is the set of points of each 1 -facet of each edge of each tile in a tiling in $(T \Gamma \sigma \Gamma \mathcal{S})$.

These maps allow us to define explicitly a labeling $\lambda$ of any tiling in $(\mathcal{T} \Gamma \sigma \Gamma$ $S$ ): Recall we described $\lambda^{-1}(\partial)$ above. The remaining points are unambiguously in the interiors edge tiles Tvertex tiles and small tiles corresponding to edges in the tiling $\Gamma$ vertices in the tiling $\Gamma$ and level- 0 supertiles. Label each point in an edge-tile with the image under $\lambda_{\mathcal{E}}$ of the closest point in the out-side of the

\footnotetext{
${ }^{8}$ We could actually do away with the other rules entirely, for our labels can all be converted into a set of bumps and nicks that fit together only when the corresponding labels match.
} 
edge-tile (or by $\partial$ if this is ambiguous) $\Gamma$ its 1 -facets with their images under $\lambda_{\mathcal{F}}$ Teach small tile with its image under $\lambda_{\mathcal{Q}}$ and markings at the curved edges derived as described in Section 3.4 (determined by the header of the label in $\mathcal{Q}) \Gamma$ and each vertex tile with the edge packets and orientations in $\mathcal{F}$ matching the edge tiles and small tiles to which it is incident.

Note every supertile can only be marked in finitely many ways $\Gamma$ since each supertile can only be marked in finitely many places and their are only finitely many possible markings. So this procedure does in fact provide a labeling of $(\mathcal{T} \Gamma \sigma \Gamma S)$.

Note we marked infinite edges with the null marking $\Gamma$ and any infinite vertex wires carry the null supertile key.

As mentioned in Section 1.3 we have now defined our well-formed supertiles $\Gamma$ configurations of tiles in $\mathcal{T}^{\prime}$ that are precisely the possible labeled supertiles in tilings in $(T \Gamma \sigma \Gamma \mathcal{S})$.

\subsection{Big tiles}

We must finesse a certain point and define our final matching rule.

Edge tiles lying on $k$-level edges $\Gamma k \geq \kappa-1 \Gamma$ of a tiling in $(T \Gamma \sigma \Gamma \mathcal{S})$ have been given a single marking $\Gamma$ but lower level edge tiles might have two or more $\Gamma$ changing at positions corresponding to skeletons of level less than $\kappa$ crossing into the tiles themselves $\Gamma$ or to sites for 0-level skeletons in the interiors of the tiles.

In some sense $\Gamma$ this issue arises because our structures really are arbitrarily fine $\Gamma$ but the tiles impose a certain "resolution" at which we view them. We simply impose a final matching rule resolving this issue:

The big tile matching rule: every tile in every tiling in $\left(\mathcal{M}, \mathcal{T}^{\prime}\right)$ must lie in a unique well-formed supertile of level $\kappa$

We call these well-formed supertiles of level $\kappa$ big tiles.

Now we have defined $\mathcal{M} \Gamma \mathcal{T}^{\prime}$ and $\left(\mathcal{M} \Gamma \mathcal{T}^{\prime}\right)$; every tiling in $(\mathcal{T} \Gamma \sigma \Gamma \mathcal{S})$ can be parsed into a tiling in $\left(\mathcal{M} \Gamma \mathcal{T}^{\prime}\right)$; we next attempt to show the converse. 


\section{The proof of Theorem 1}

We turn now to the space $\left(\mathcal{M} \Gamma \mathcal{T}^{\prime}\right)$ of matching rule tilings. With all this work behind us $\Gamma$ the task is relatively simple:

Recall from Section 1.3 we defined a well-formed $n$-level supertile to be a configuration of tiles in $\mathcal{T}^{\prime}$ that is the image of a supertile in some labeling.

That is $\Gamma$ in a well-formed supertile $\Gamma$ we do not need to be too careful about whether this supertile lies in a labeled tiling in $(T \Gamma \sigma \Gamma \mathcal{S})$ or in a tiling in $\left(\mathcal{M} \Gamma \mathcal{T}^{\prime}\right)$ : our well-formed supertiles have skeletons $\Gamma$ sites and wires clearly marked $\Gamma$ and these structures carry packets that are consistent across the entire configuration.

We are to show that every point in the interior of a tile in an tiling in $\left(\mathcal{M} \Gamma \mathcal{T}^{\prime}\right)$ lies in a unique $n$-level well formed supertile for each level. We would like to induct. However $\Gamma$ this is not quite possible: in an induction $\Gamma$ we cannot be sure of the consistency of the packets for a particular skeleton until the skeleton is completely formed; however $\Gamma$ because a skeleton may span several levels $\Gamma$ there may be a lag of several steps of the induction between a skeletons first appearance and the point at which it is connected. Thus $\mathrm{w}$ we define instead:

A configuration $C$ of tiles in $\mathcal{T}^{\prime}$ is an almost-well-formed $n$-level supertile there is a congruence $\mathrm{B} \in \mathcal{G}$ to a well-formed $n$-level supertile $C^{\prime} \Gamma$ such that every tile in $C$ is congruent under $\mathrm{B}$ to a tile in $C^{\prime}$; and such that for any packet labeling a tile in $C \Gamma$ all information of level $n$ or lower is the same in the packet of the corresponding tile in $C^{\prime}$. That is $\Gamma$ an almost-well-formed $n$-level supertile differs only from a well-formed n-level supertile in that the consistency of information concerning higher level structures has not yet been checked for.

Specifically we note the $(n-1)$-level edges of the almost-well-formed supertile must all share the same packet $P$ because of the compatibility rules and the fact that the skeleton of the almost-well-formed supertile is connected. Because the supertile is well-formed $\Gamma$ it is clear which edges on the boundary of the almostwell-formed supertile are meant to lie in the interior of the supertile's parent; moreover $\Gamma$ on each such edge there is exactly one site serving the supertile $\Gamma$ a marking at a vertex-tile $v$ on the supertile's boundary; moreover $\Gamma$ all vertices $\sigma^{n}(v) \Gamma v \in \mathcal{V}(\mathrm{B})$ such that $v$ is incident to an edge in $E(\mathrm{~A})$ are either meso- or endo- vertices of $\sigma^{(\mathrm{A})}$ and are unambiguously identified with either a terminal or a vertex in $\mathcal{V}^{\prime \prime}(\mathrm{A})$.

Since our big tiles are themselves almost well-formed supertiles of level $\kappa \Gamma$ the following proposition $\Gamma$ with Lemma $4.2 \Gamma$ completes an inductive proof of the Theorem. 
Proposition 4.1 If any almost well-formed supertile of level $j, j \leq n$ lies in an almost well-formed supertile of level $n$, every almost well formed supertile of level $j, j \leq n$ lies in an almost well-formed supertile of level $n+1$.

Proof We proceed by induction and may assume that $n>\kappa$ and that every tile lies in an almost well-formed supertile of each level $k<n$.

But we have gone to a great deal of trouble already: the work is already done! Consider an almost well-formed $n$-level supertile $\Gamma$ congruent to $\sigma\left(\mathbf{X}_{1}\right)$ for some $\mathrm{X}_{1} \in \mathcal{S} \Gamma$ and let $\mathrm{X}_{2}$ in $\mathrm{X}_{1}^{-}$(figure 6 ). We must show our almost wellformed supertile lies in an almost well-formed supertile congruent to $\sigma^{(n+1)}\left(\mathrm{X}_{2}\right)$. We must check that our original supertile and its siblings form the correct configuration and that the markings on the skeleton and vertex wires of of $\sigma^{(n+1)}\left(\mathrm{X}_{2}\right)$ are correct.

Suppose an almost well-formed supertile has supertile key $Q=\left[X_{\kappa} \ldots X_{1}, \ldots\right] \in$ $\mathcal{Q}$. At each site $\sigma^{n-1}(z)$ serving the skeleton of the supertile the compatibility rules (Section 3.3) ensure that immediately above the site's marking is a vertex hull in $\mathcal{V}^{\prime \prime}\left(\mathrm{X}_{2}\right)$ Twith edges marked with supertile key $\mathrm{Q}^{\prime}$ where $\mathrm{Q} \Gamma \mathrm{Q}^{\prime}$ are paired (Section 2.4). MoreoverTat this site the edges corresponding to the $n$-level edges in the skeleton of $\sigma^{n+1}\left(\mathrm{X}_{2}\right)$ are all correctly labeled and oriented.

Now the matching rules ensure that at least next to $v$ Tthe appropriate edges of the parent skeleton set forth in the right orientations Tfollowing the boundary of our $n$-level supertile.

The edge must propagate along the boundary of the $n$-level supertile Taided by vertices with overpass markings. Note that as the edge propagates $\Gamma$ the higher level markings on the vertices of the $n$-level supertile are correlated with one another by the edge matching rule (Section 3.5).

The edge will propagate along the $(n-1)$ face of the underlying polyhedron until meeting vertex markings in $\mathcal{V}^{\prime \prime}\left(\mathrm{X}_{2}\right)$. But these markings have been anticipated and lie at fixed locations on the boundary of $\sigma\left(\mathrm{X}_{1}\right)$. That is $\Gamma$ the edge cannot propagate beyond its intended boundaries nor fall short of these boundaries $\Gamma$ as these vertices are fixed.

Because the original tiling is sibling edge-to-edge $\Gamma$ these terminals must be terminals for the edges of neighboring supertiles; moreover $\Gamma$ these edges must convey the same trailer of the packet in $\mathcal{P}$. However $\Gamma$ the nature of these neighboring supertiles $\Gamma$ and their precise alignment is not yet determined. 
In particular Twe must eventually come across the site lying to the other side of the edge; this site must be adjacent to some big tile; this big tile must lie in some well-formed $n$-level supertile. Since this supertile must share the same vertices as $\sigma^{n}\left(\mathrm{X}_{1}\right)$ Tits position and nature are fixed: it is indeed the appropriate sibling of $\sigma^{n}\left(\mathrm{X}_{1}\right)$ Tin the correct position.

Now note that information concerning level higher than $n$ supertiles in the packet in the tiles in $\sigma^{n}(\mathrm{~A})$ and $\sigma(\mathrm{C})$ must be correlated by this edge. And so we can walk our way about $\Gamma$ showing each sibling in turn is in its place.

Finally $\Gamma$ because the skeleton $E_{\mathrm{X}_{2}}$ is connected $\Gamma$ we have that the supertile key is consistent across all of $\sigma^{n+1}\left(\mathrm{X}_{2}\right)$; moreover all structures are correctly placed and oriented. Thus Tour original almost well-formed supertile of level $n$ lies in an almost well-formed supertile of level $n+1$.

$$
\text { qed }
$$

The following lemma completes the proof of the theorem.

Lemma 4.2 If every point in the interior of every tiling in $\left(\mathcal{M}, \mathcal{T}^{\prime}\right)$ lies in a unique almost well-formed supertile of each level $n \in \mathrm{N}$ then every point in the interior of every tiling in $\left(\mathcal{M}, \mathcal{T}^{\prime}\right)$ lies in a unique well-formed supertile of every level $n$.

Proof This is simply because any almost well-formed supertile of level $n$ in an almost well-formed supertile of level greater than $n+\kappa$ is in fact a well-formed supertile.

qed

We used the condition in the statement of the theorem very strongly: we needed a mechanism - vertex wires - to fix the position of the vertices of $\sigma(\mathrm{A})$ and thus keep edges from propagating beyond their intended borders. The vertex wires required vertices to be hereditary. Secondly to fix the position of sibling supertiles relative to some initial supertile $\Gamma$ we need some point in each we can say they share- they are forced to share vertices.

Other mechanisms can be devised $\Gamma$ exploiting other conditions one could make. However it is not clear if an example exists in which the relative positions of sibling tiles cannot be fixed at all.

Note that we have specifically shown that every tile in an $\mathcal{M}$ - tiling lies in 
arbitrarily large well-formed supertiles. This is the definition we have taken for enforcement. Note though $\Gamma$ that if the tiling in $\left(\mathcal{M} \Gamma \mathcal{T}^{\prime}\right)$ has an infinite fault $\Gamma$ we have no control over the edge-label propagated down the fault; similarly a vertex wire might serve no highest level supertile. We also have no control over slippage along such a fault. In the vertex to vertex case $\Gamma$ we can stiffen this structure up considerably.

In Appendix $\mathrm{C}$ we point out that the correspondence between the tilings in $\left(\mathcal{M} \Gamma \mathcal{T}^{\prime}\right)$ and tilings in $(\mathcal{T} \Gamma \sigma \Gamma \mathcal{S})$ is one-to-one $\Gamma$ except on a set of measure zero in any translation invariant probability measure on $(\mathcal{T} \Gamma \sigma \Gamma \mathcal{S})$.

We also reiterate that $\Gamma$ given a substitution tiling $\Gamma$ we produce a tiling with matching rules that encode the addresses generated by the substitution system. If the addresses do not correspond to unique substitution tilings - in particular $\Gamma$ if the substitution tilings can be periodic- then our correspondence with $(\mathcal{M} \Gamma$ $\mathcal{T}^{\prime}$ ) cannot be exact. Also $\Gamma$ if the tiles have some particular symmetry algorithm produces tiles in which the symmetry has been broken. 


\section{A Survey of substitution tilings}

We first give a quick history of the problem $\Gamma$ then survey a variety of substitution tilings Tmany of which have not been previously published. This appendix may give the reader a feel for some of the technical issues that arise in the construction.

\section{A.1 History}

If a set of prototiles admits no tiling that is invariant under an infinite group of congruences in our space $\Gamma$ the prototiles are aperiodic. The possibility of an aperiodic set of prototiles $\Gamma$ in the plane or elsewhere $\Gamma$ was essentially ignored until H. Wang and R. Berger began investigating connections between tilings and undecidability.

In the early 1960's Wang encoded arbitrary Turing machines as tilings of the plane. However he required a somewhat awkward restriction- the placement of a "seed" tile to begin the computation. In 1964 Berger removed this restriction by constructing an underlying hierarchical structure ${ }^{9}$; this structure provided arbitrarily large $\Gamma$ enclosed domains for the run of the machine [Ber].

Berger thus could answer that the "Domino Question" is undecidable; that is $\Gamma$ there is no an algorithm to decide whether any given set of prototiles admit a tiling. Berger's tiles admitted a tiling if and only if the underlying Turing machine did not halt.

If every set of prototiles admitting some tiling admits a periodic tiling Tone can produce an algorithm to check whether any given set of prototiles actually admits a tiling. Thus Berger produced an unexpected corollary- there are sets of prototiles that do tile the plane Cbut cannot do so aperiodically! He gave such a set of some 201000 tiles.

By 1971 Robinson had produced a much smaller set of six prototiles and streamlined Berger's general result as well in an especially lovely and readable paper [Rob]. R. Penrose found his celebrated tiles in 1972 [Pen] and many other sets have been found since. R. Amman in particular has contributed many beautiful examples [GrSh].

\footnotetext{
${ }^{9}$ Interestingly, Berger called this structure a "skeleton".
} 
N.G. De Bruijn pointed the way to another interpretation in 1981: aperiodic tilings could arise as projections of slices through a higher dimensional lattice $[\mathrm{dBr}]$. This "slice and project" method of constructing aperiodicity has been very much studied. Recently matching rules have been given for a wide class of such tilings [Le]. One wonders if every slice-and-project tiling enforceable by matching rules has a hierarchical structure.

In 1984 physical materials were found sharing the peculiar Fourier spectrum of aperiodic tiles with icosahedral symmetry studies. An enormous amount of inquiry into possible structures for these materials was undertaken (cf. [StO $] \Gamma[\mathrm{Luc}])$. M. Senechal has provided a readable and comprehensive survey [Sen].

Robinson's set of prototiles enforced aperiodicity by ensuring a specific structure was formed: an infinite hierarchy of similar square lattices in the plane (figure 15). The collection as a whole is aperiodic $\Gamma$ since no translation can leave every scaled lattice invariant.

Since then $\Gamma$ virtually every set of aperiodic prototiles produced either has made use of such a hierarchical structure to ensure aperiodicity $\Gamma$ or has been derived by the slice-and-project method with the notable exception of the recent Schmitt-Conway-Danzer tiling [Dan].

Often this hierarchical structure is produced through a substitution system. A standard method of showing that a set of prototiles is indeed aperiodic is to show the set enforces a uniquely decomposable substitution structure.

Although Robinson's original tiles form hierarchical tilings $\Gamma$ they do not overtly arise from a substitution process. They can $\Gamma$ however $\Gamma$ be parsed into a substitution tiling $\Gamma$ as described in [GS].

We would like to give a variety of two TthreeTand higher dimensional examples of substitution tilings. All of these fall within the realm of our Theorem $\Gamma$ and can be enforced by matching rules. Clearly substitution tilings are abundant; it is not known if there are uncountably many combinatorially distinct substitution tilings.

The substitution tiling on the left of figure 16 is periodic and does not have unique decomposition.

However Tif we mark our tiles $\Gamma$ we may find aperiodicity and unique decompo- 


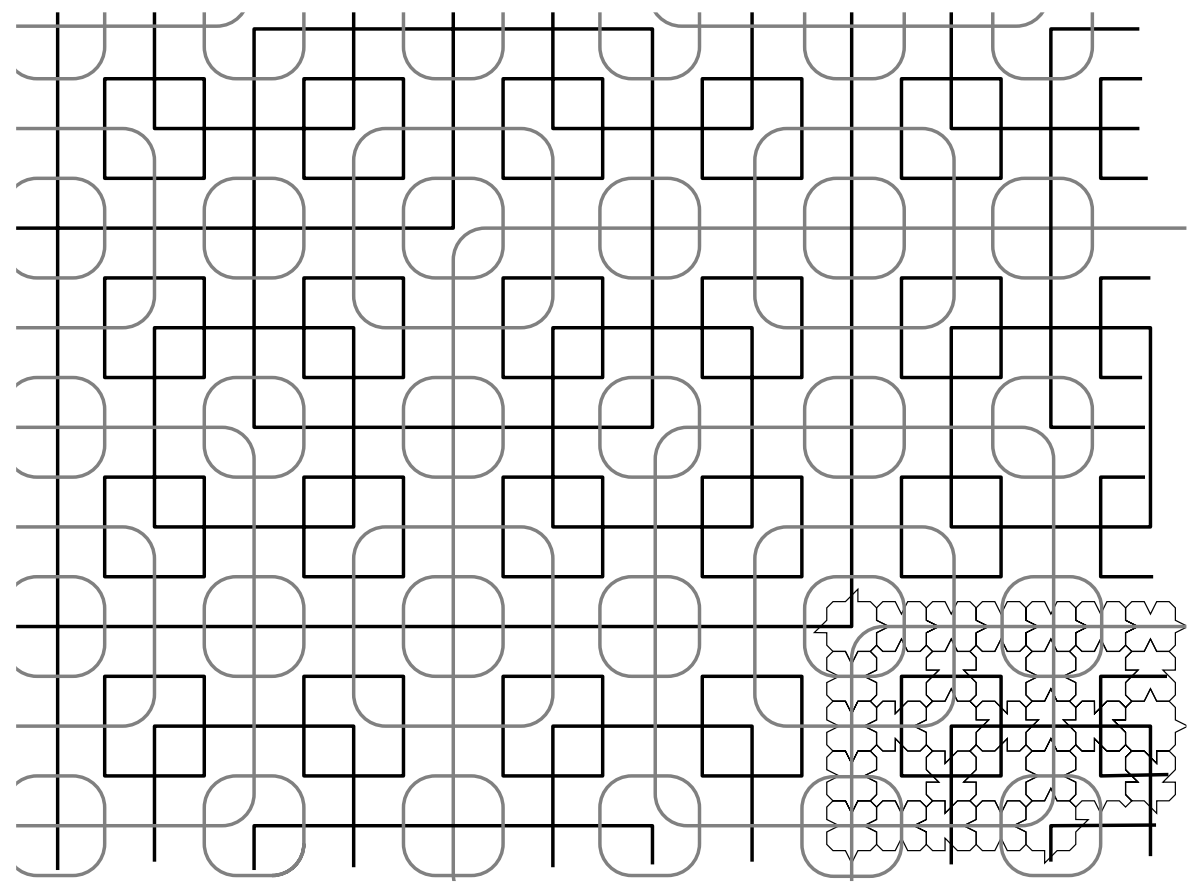

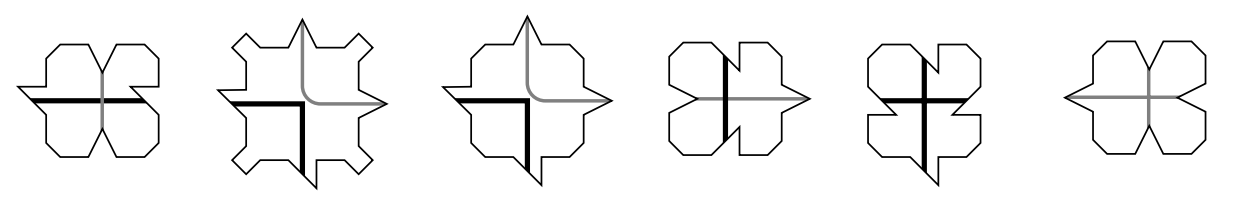

Figure 15: An aperiodic collection of square lattices and Robinson's tiling 
sition! Eight different congruences could have used to place each daughter tile; any gives periodic structures if the tiles are unmarked $\Gamma$ but generically $\Gamma$ marked tiles should give aperiodic structures. The tiling on the right of figure 16 is aperiodic and does have unique decomposition.
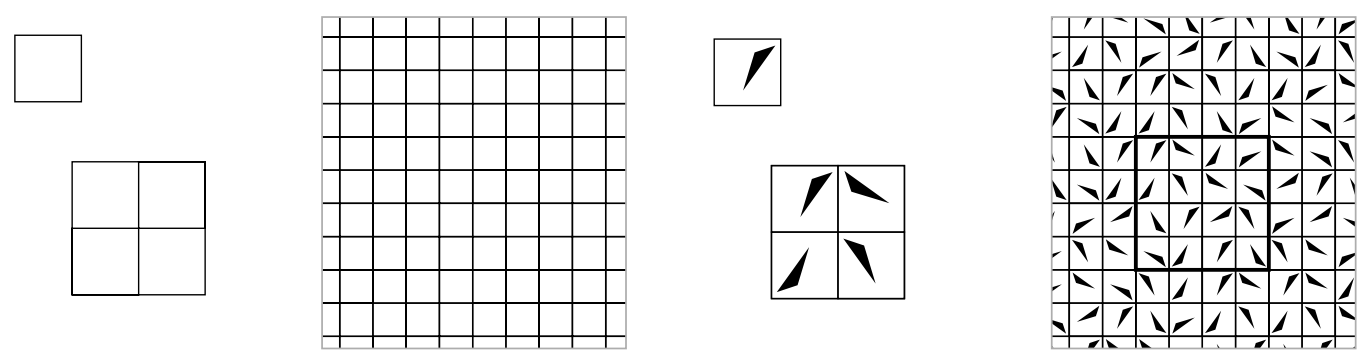

Figure 16: Two quite distinct substitution tilings

\section{A.2 Well known examples}

In figure 17 are a number of well known examples $\Gamma$ clockwise from upper-left: the "Sphinx" $\Gamma$ discussed at length in [Godr] with matching rules provided by E.A. Robinson [RobE]; the "Pinwheel" $\Gamma$ found by Conway with matching rules provided by Radin [Rad]; a "Dimer" tiling $\Gamma$ with matching rules found by E.A. Robinson; and the "Half-hex" $\Gamma$ with matching rules provided by Socolar [Soc].

Note that the pinwheel can be generalized in many ways: one family of tilings has for every unordered pair of natural numbers $p, q 2^{\left(\left(p^{2}-p+q^{2}-q\right) / 2\right)}$ distinct "pinwheel-like" tilings with right triangles with legs of length $p, q$ and inflation by a factor of $\sqrt{p^{2}+q^{2}}$. (Here $p, q=2,1$ ). L. Sadun [Sad] has given a generalization parameterized by the rational numbers (in fact $\Gamma$ he corresponds the irrationals to tilings too but requires infinite congruence classes of prototiles in this case).

\section{A.3 Triangles}

Triangles provide many interesting examples. The first example in figure 18 is due to Robinson and is equivalent to the Penrose tiling. 

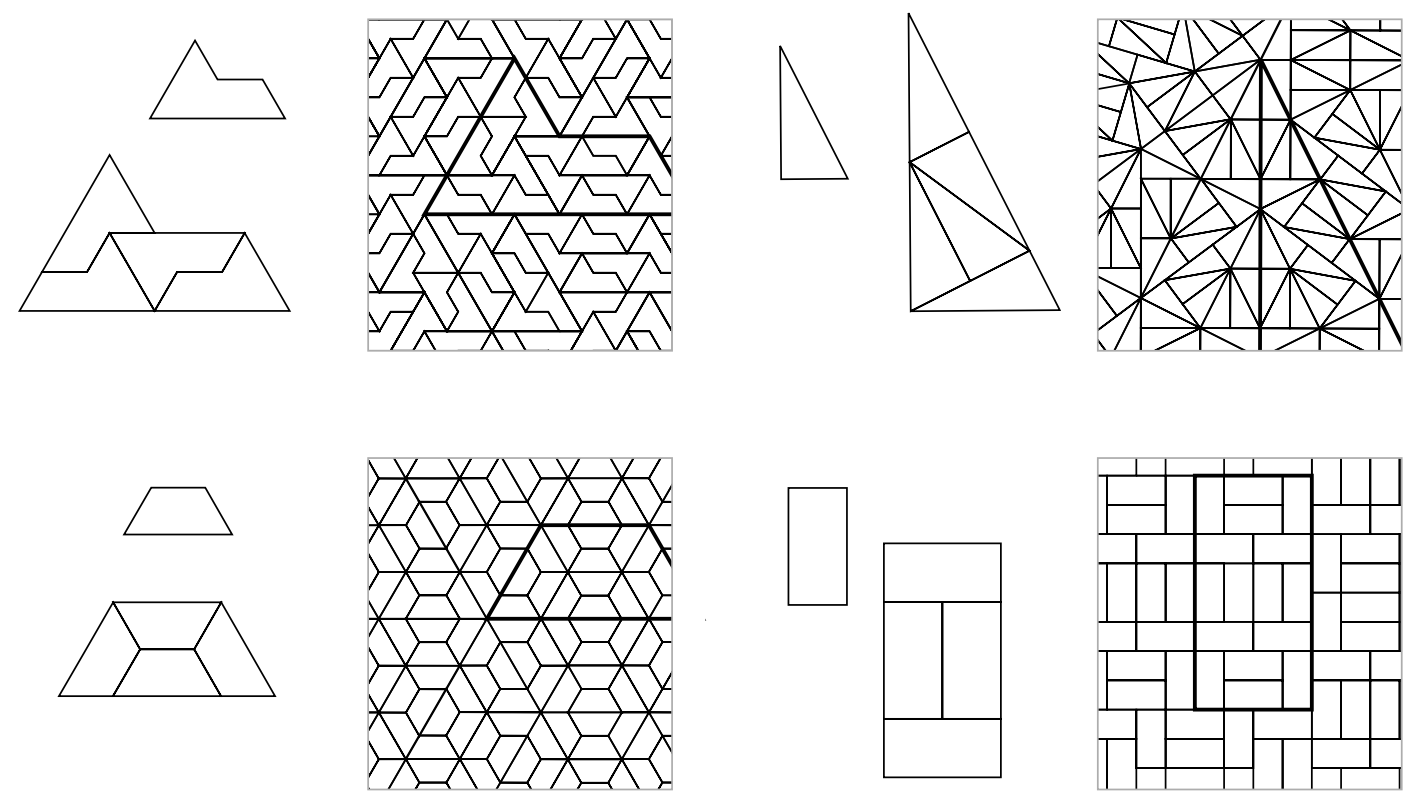

Figure 17: The Sphinx Tthe Pinwheel $\Gamma$ the Half-Hex and the Dimer 
The example at upper right is due to the author; the modulus of the inflation is the real root of $s^{3}-s-1$. Danzer has generalized this: for each $j, k$ there is one real solution $s>1$ of $s^{(2 j+k)}-s^{j}-1$; each such root is the modulus of an inflation for a family of tilings [Dan]. In particularTin each case there is one free $\Gamma$ real parameter (although in the case $j=k=1$ explored by the author $\Gamma$ this parameter gives only finitely many combinatorially distinct tilings.)

The example at lower left is due to Danzer [Dan]; in this particular variation the tiles appear in an infinity of orientations $\Gamma$ as with the pinwheel. A similar example will be examined more closely in Appendix B. The final example is a simple variation of the third: one congruence in the substitution rule has been reflected.

Danzer has also produced an infinite family of triangle substitution tilings with inflation through the roots of $s^{k}-s^{2}-1$ Tas well as many other beautiful examples. All in all This suggests that perhaps this is the palest shadow of the full situation.
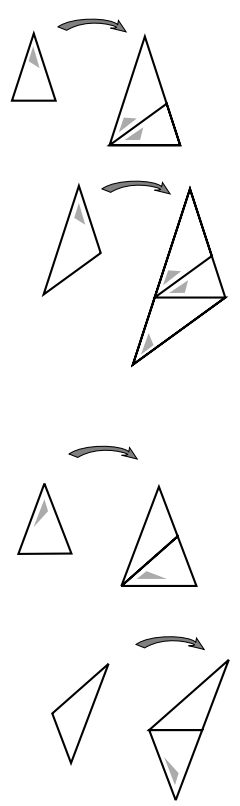
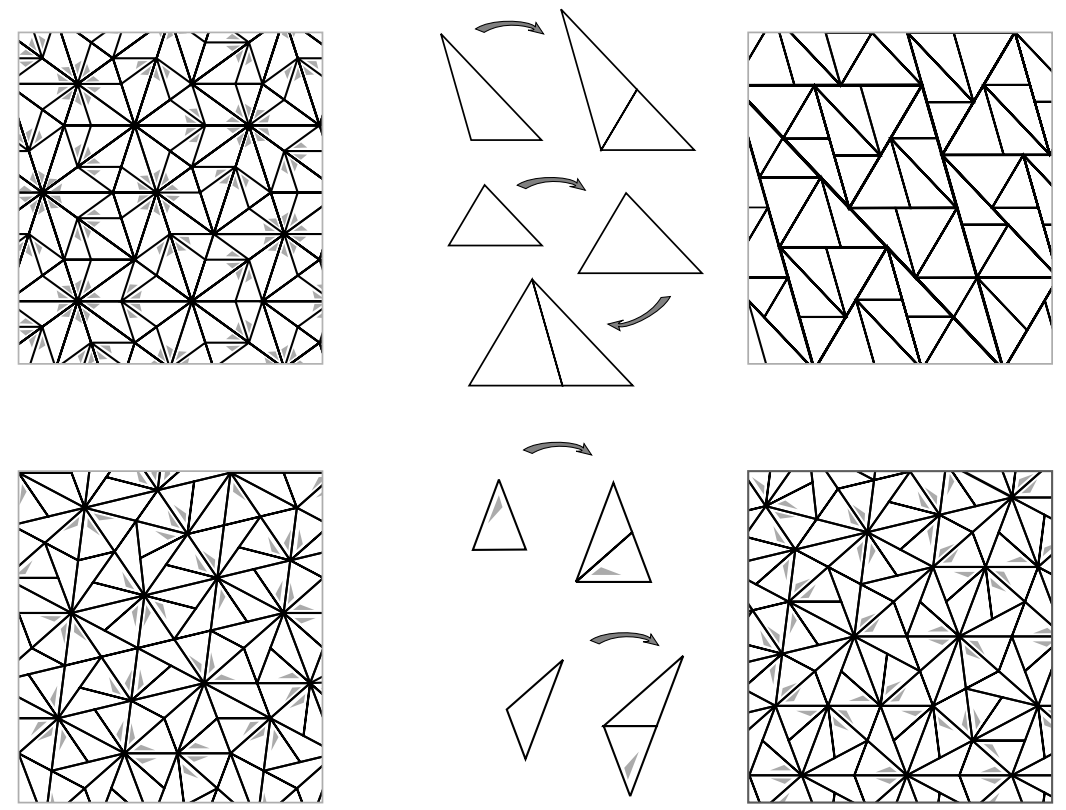

Figure 18: Four substitution tilings with triangles 


\section{A.4 Higher dimensional examples}

Multidimensional examples should be in even richer abundance. In figure 19 is one example; simple matching rules were given in [GS]. Cubes oriented in varying directions $\Gamma$ in the mode of the example in figure 16 Tand products of symbolic substitution systems $\Gamma$ as in figure 20 Talso give families of examples in $E^{n}$.

Radin and Conway recently constructed an interesting example [Rad2]; the orientations of the tiles are dense in $\mathrm{SO}(3)$. Examples such as this should be generic $\Gamma$ as is suggested by the known infinite families of the triangle tilings in the plane.

The author knows of one special example in $E^{4} \Gamma$ based on the stellations of the 120-cellГand a few three dimensional examples $\Gamma$ but for the time being $\Gamma$ the higher dimensional terrain is basically unexplored. It is unknown even if a single similarity class of tetrahedron can serve as prototiles in an aperiodic substitution tiling. Such an example would be very interesting.
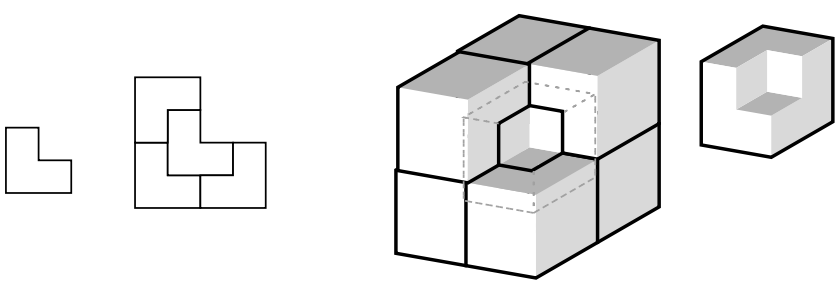

Figure 19: An example in each $E^{n} \Gamma n>1$

\section{A.5 Products of symbolic dynamical systems}

Symbolic dynamics provides examples readily; Mozes gave matching rules for tilings derived as the product of two one-dimensional symbolic substitution systems [Moz]. Clearly such substitution tilings can be generalized to arbitrary dimension. Note that Mozes actually used square tiles with non- homogeneous inflation. However $\Gamma$ Kenyon [Ken] points out that any one dimensional symbolic substitution system is isomorphic to a substitution tiling in $E^{1}$ Tacting on line segments of varying lengths. Thus we can adjust Mozes' tiles- the product of such 1-dimensional systems- making them marked rectanglesГsuch that an expanding linear map acts on the tiles. 

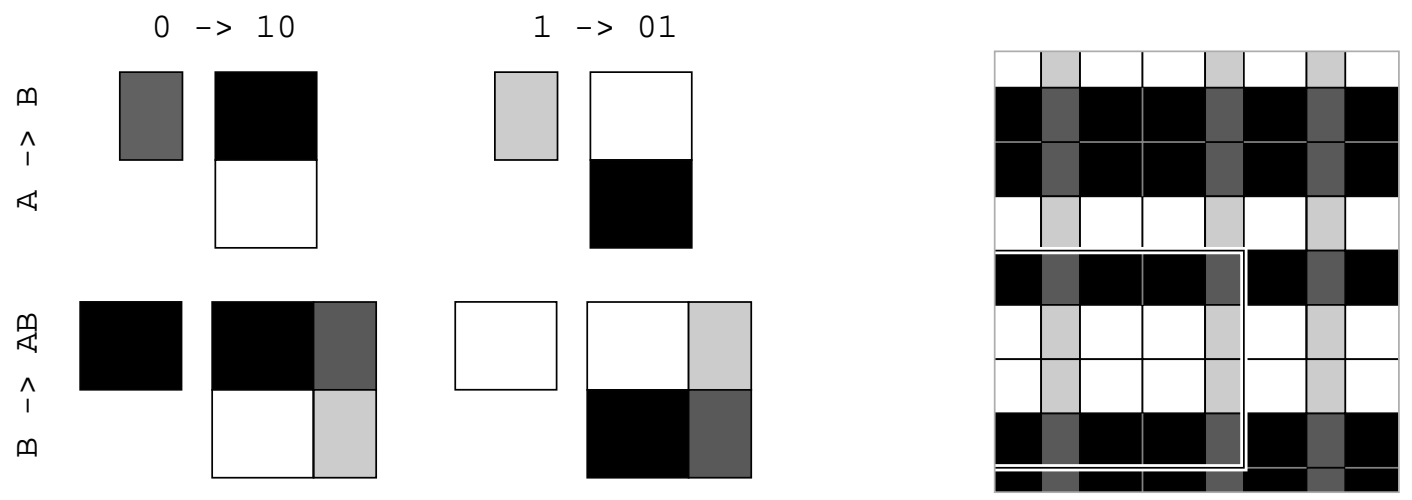

Figure 20: A product of one dimensional substitutions

\section{A.6 Non- vertex-to-vertex examples}

An important notion is that of vertex-to-vertex. A substitution tiling is vertexto-vertex if there is a discrete set of vertices for the prototiles such that if vertex of one tile meets another tile $\mathrm{Cit}$ is coincident with a vertex of the other tile. Radin points to the "Pinwheel Sandwich" as an example of a tiling that cannot be vertex-to-vertex $[\operatorname{Rad} 2]$. Two versions of the pinwheel are layered; on the right in figure 21 is an illustration of how part two such layers meet after only three substitutions- the situation already seems out of hand! The pinwheel sandwich does meet the condition in our Theorem however.

L. Sadun [Sad] has given a substitution tiling in $\mathrm{E}^{2}$ he can prove is not vertex-to-vertex. Danzer maintains there are many 2-dimensional examples of substitution tilings that cannot be vertex-to-vertex.

One hopes to know eventually how $\Gamma$ given a substitution tiling $\Gamma$ one can produce a set of vertices if there is oneГor anticipate that there should be a set of vertices Tor prove that there can be no set of vertices such that the tiling is vertex-to-vertex.

\section{A.7 Unique decomposition}

A substitution tiling has unique decomposition if every tiling under the substitution has a uniquely defined hierarchy. Clearly if a tiling is periodic it cannot have a unique hierarchy but until very recently nothing was none about the converse. 

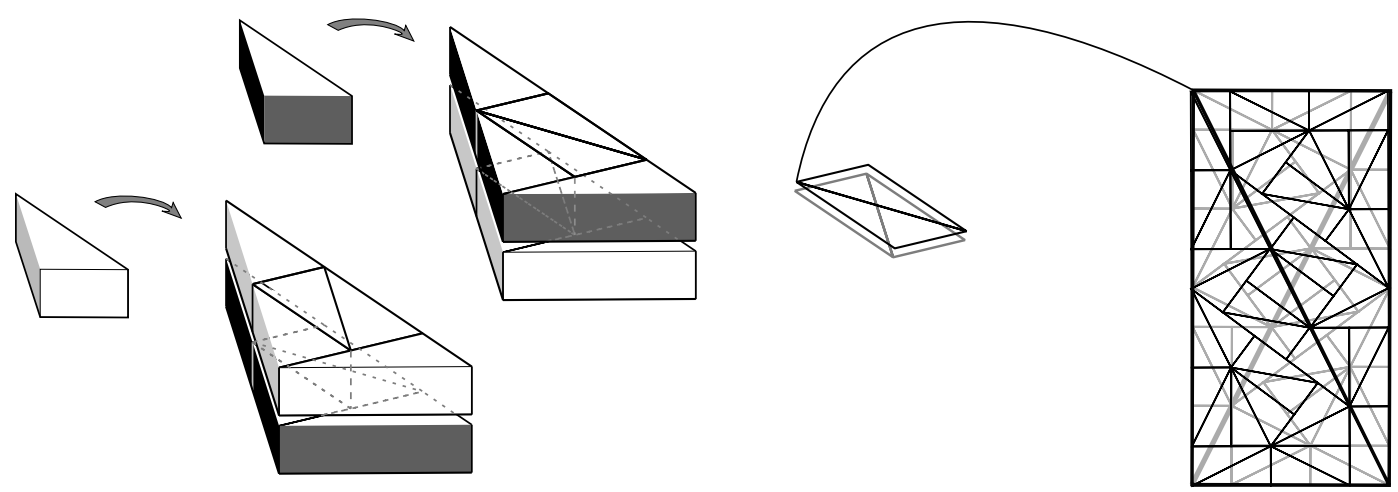

Figure 21: The "Pinwheel Sandwich" Tnot vertex-to-vertex

B. Solomyak [Sol] recently showed that - if the tiles have exactly as much symmetry as the supertiles- then non-unique decomposition implies the substitution tiling is periodic. His result is sharp with respect to this condition:

The degenerate example of figure 22 is aperiodic and has non-unique decomposition if the tiles are unmarked; however the tiles have more symmetry than the supertiles in this case. The same tiling with fully marked tiles is uniquely decomposable. The non-uniqueness arises because there are two reasonable ways to mark the tiles in the tiling- each leading to a different decomposition.

To show the congruences in the substitution rules $\Gamma$ we must very lightly mark the tiles. However in the tiling $\Gamma$ we give the unmarked tiles and indicate two possible supertiles.

\section{A.8 Out of our realm}

We should examine some tilings that deserve to be called substitution tilings but are not addressed by our theorem.

First $\Gamma$ we need that our tiles have only finite congruence classes $\Gamma$ in order to have finite classes of labels. There might be two natural generalizations: (a) simply allow the matching rules to have the same cardinality as the set of prototiles (but retain the requirement that they be localГe.g. bounded $\Gamma$ so that there is still substance to the enforcement). In this case $\Gamma$ the techniques of this paper should give a strong analogue to our main theorem. (b) one might allow finite similarity classes and infinite congruence classes. In this second case techniques give a strong analogue to the theorem only if the scales of similar 

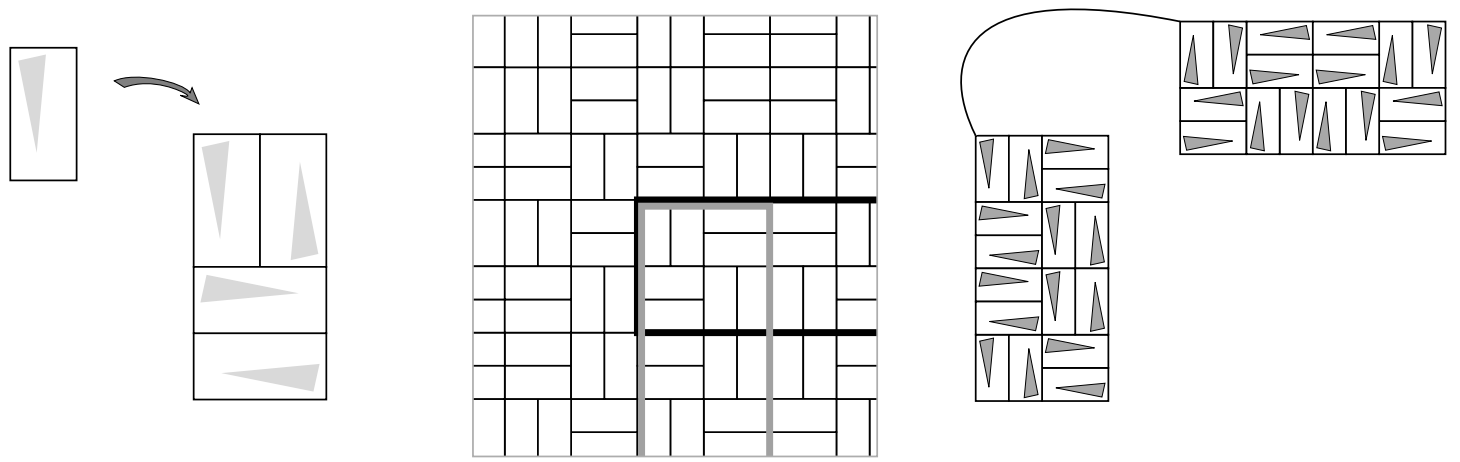

Figure 22: An aperiodic example with two decompositions

prototiles lie within some narrow range; otherwise one cannot control how finely a particular well-formed supertile is divided.

There are many examples of substitution tilings for both generalizations. Stellations of polytopes $\Gamma$ in particular $\Gamma$ naturally lead to substitution tilings; usually these require a finite collection of similarity classes of tile $\mathrm{C}$ but an infinite collection of congruence classes. For example $\Gamma$ a construction based on the stellations of the heptagon is given in figure 23 (rules are given for each similarity class).

L. Sadun has given a different $\Gamma$ uncountable collection of tilings falling into this category [Sad].

Second $\Gamma$ we need that our tiles are polyhedra; this is so that we can be sure to have various structures to enlist for transporting information- edges to carry information from one place to another and vertices to keep the local structure of our information well formed.

Thurston [Thu] and Kenyon [Ken] have given many interesting examples of self-similar tilings for which the tiles are decidedly not polyhedral; furthermore $\Gamma$ although these tilings all allow a single similarity class of tile $\Gamma$ many require infinitely many congruence classes of tile $\mathrm{Tand}$ so are doubly disqualified. However $\Gamma$ it seems very likely that the techniques we use can be extended to cover this type of tiling- for the primary properties of edges are that they are selfsimilar the vertices are discrete and at rational points; indeed the boundaries of Thurston and Kenyon's tiles admit analogues to both vertices and edges.

A very famous example- the Penrose tiles- does not fall into the category we describe herein $\Gamma$ for the inflated tile is not strictly congruent to the config- 

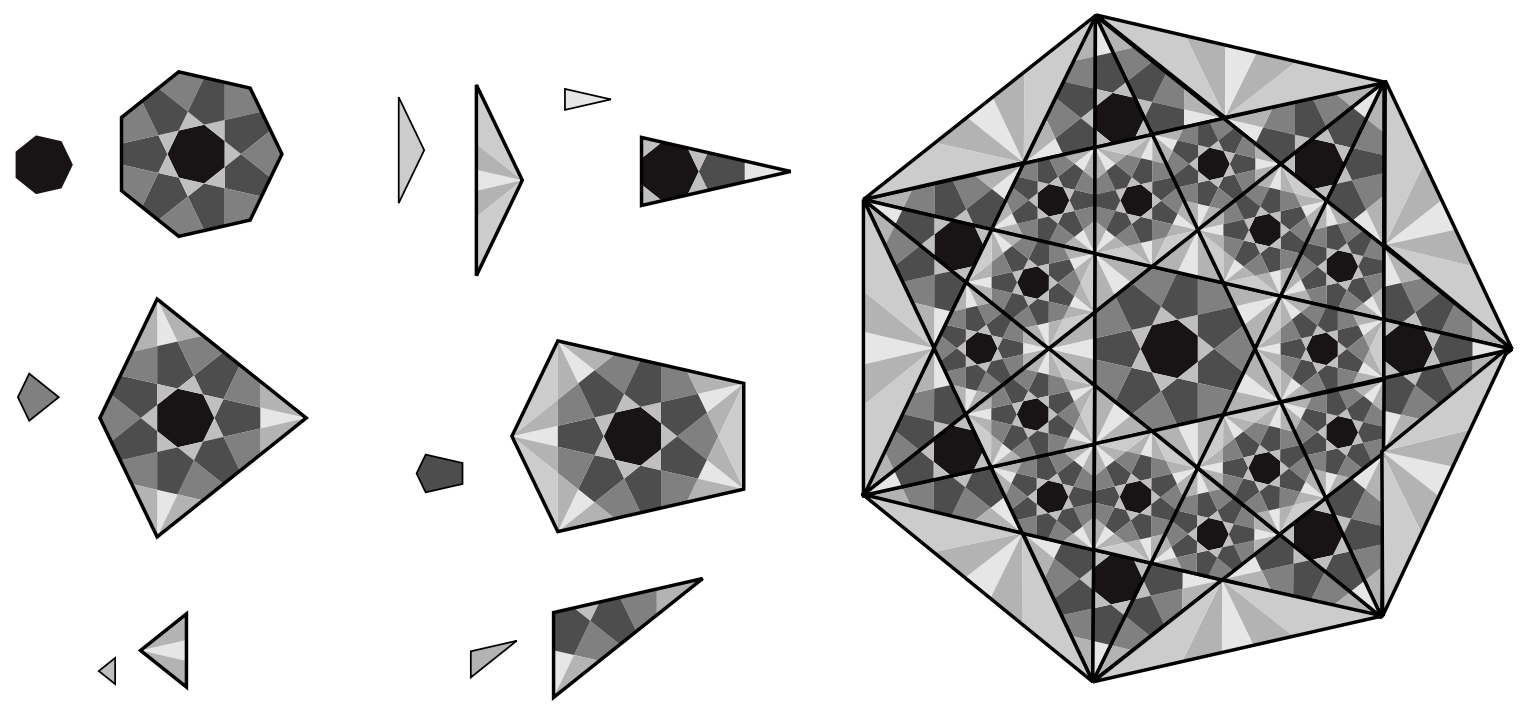

Figure 23: Not a substitution tiling Tfor our purposes

uration of daughter tiles replacing it (figure 24). However Tthe Penrose tiling is equivalent to the substitution tiling by Robinson triangles (figure 18) $\Gamma$ and it seems exceedingly likely that if this sort of more general substitution yields tilings that are vertex-to-vertex $\Gamma$ then the tiles can be recomposed into tiles residing in our setting. (Also in vertex-to-vertex tilings $\Gamma$ a much stronger notion of enforcement may be taken: slipping along infinite faults can be eliminated).
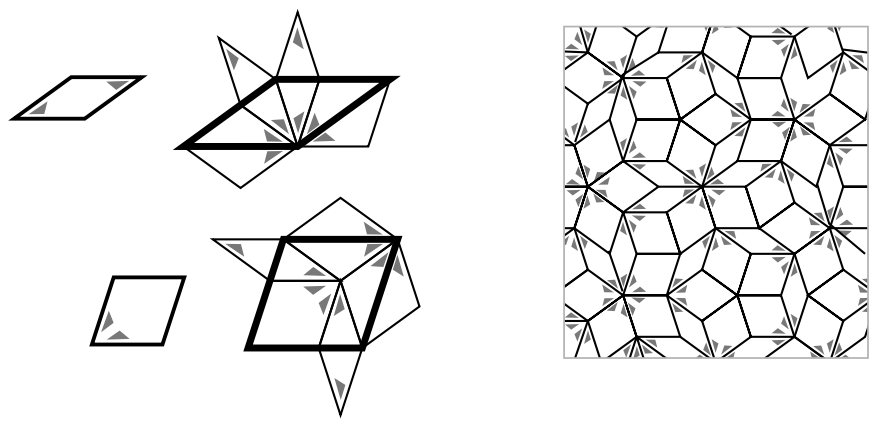

Figure 24: Not a substitution tilingTfor our purposes 


\section{B A simple example}

We illustrate the construction with a variation of Danzer's tiling from the lower right of figure 18; this example has the distinction of being one of the very simplest possible examples with which to work. (This is because mostly because $\mathcal{S}$ is small $\Gamma$ the skeletons are exceedingly simple $\Gamma$ and $\kappa=1$.)

We take the substitution illustrated below. In figure $25 \Gamma \mathcal{T}=\{\mathrm{X}, \mathrm{Y}\} \Gamma \mathcal{S}=$ $\{\mathrm{A}, \mathrm{B}, \mathrm{C}, \mathrm{D}\} \Gamma \sigma \Gamma \sigma^{6}(\mathrm{X}) \Gamma \sigma^{6}(\mathrm{Y})$ are illustrated.
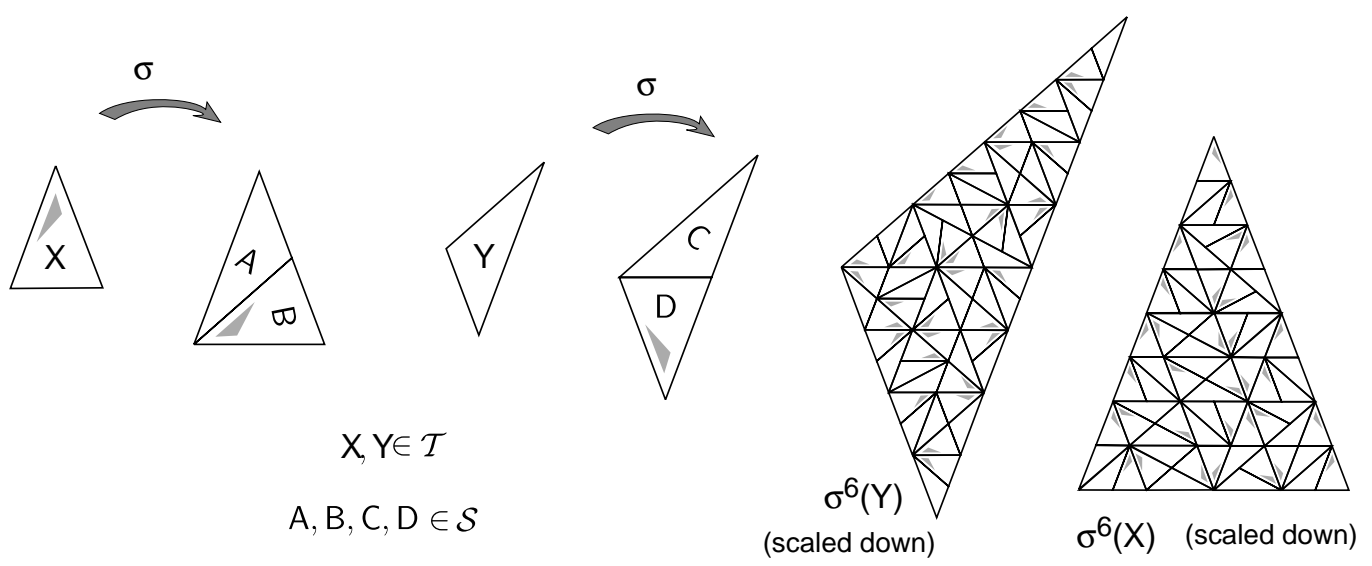

Figure 25: $\mathcal{T} \Gamma \sigma$ and $\mathcal{S}$

We next begin defining structures for the construction (figure 26). As in Section 2.1.1 we define

$$
\begin{aligned}
& \mathcal{V}(\mathrm{A})=\{1 \mathrm{~A}, 2 \mathrm{~A}, 3 \mathrm{~A}\} \Gamma \\
& \mathcal{V}(\mathrm{B})=\{4 \mathrm{~B}, 5 \mathrm{~B}, 6 \mathrm{~B}\} \Gamma \\
& \mathcal{V}(\mathrm{C})=\{1 \mathrm{C}, 2 \mathrm{C}, 3 \mathrm{C}\} \Gamma \\
& \mathcal{V}(\mathrm{D})=\{4 \mathrm{D}, 5 \mathrm{D}, 6 \mathrm{D}\} \text { and } \mathcal{V}=\mathcal{V}(\mathrm{A}) \cup \mathcal{V}(\mathrm{B}) \cup \mathcal{V}(\mathrm{C}) \cup \mathcal{V}(\mathrm{D}) .
\end{aligned}
$$

Note that $2 \mathrm{~A} \Gamma 6 \mathrm{~B} \Gamma 4 \mathrm{D}$ Tand $1 \mathrm{C}$ are mesovertices; $3 \mathrm{~A}$ प4BГ $3 \mathrm{C}$ and $5 \mathrm{D}$ are endovertices and the rest are epivertices.

We next define: $\mathcal{E}(\mathrm{X})=\{+\mathrm{i},-\mathrm{i}\} \subset \sigma(\mathrm{X}) \Gamma \mathcal{E}(\mathrm{Y})=\{+\mathrm{ii},-\mathrm{ii}\} \subset \sigma(\mathrm{Y})$ and $\mathcal{E}=\{+\mathrm{i},-\mathrm{i},+\mathrm{ii},-\mathrm{ii}\}$.

We turn to sites (figure 27ГSection 2.1.2). Each edge meets a suitable site after only one inflation. Moreover $\Gamma$ the edges in $\mathcal{E}(\mathrm{X}) \Gamma \mathcal{E}(\mathrm{Y})$ are connected. So 

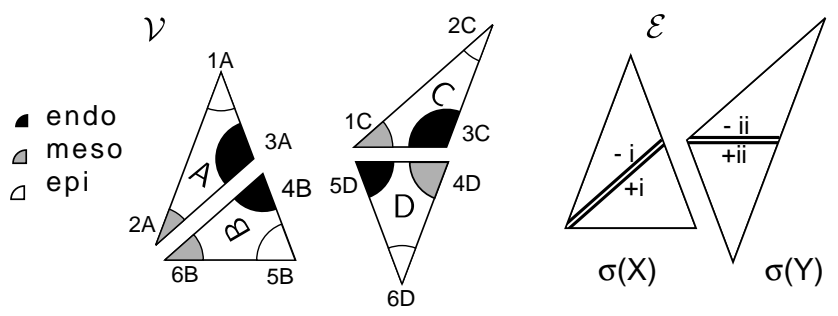

Figure 26: $\mathcal{V} \Gamma \mathcal{E}$

we are fortunate enough to have $\kappa=1(\text { Section 2.1.3 })^{10}$. We take

$$
\begin{aligned}
& \mathcal{Z}(\mathrm{A})=\{3 \mathrm{~A}=\mathrm{z}(-\mathrm{i})\} \Gamma \\
& \mathcal{Z}(\mathrm{B})=\{4 \mathrm{~B}=\mathrm{z}(+\mathrm{i})\} \Gamma \\
& \mathcal{Z}(\mathrm{C})=\{3 \mathrm{C}=\mathrm{z}(-\mathrm{ii})\} \Gamma \text { and } \\
& \mathcal{Z}(\mathrm{D})=\{5 \mathrm{D}=\mathrm{z}(+\mathrm{ii})\} .
\end{aligned}
$$

Our skeletons (Section 2.1.4) will just be the edges $\mathcal{E}$ Гbut within elements of $\sigma(\mathcal{S})$. We define the vertices and edges of the skeletons:

$$
\begin{aligned}
& \mathcal{V}^{\prime}(\mathrm{A})=\{I \mathrm{~A}, \| \mathrm{A}, \mathrm{VIA}, \mathrm{VII}\} \subset \sigma(\mathrm{A}) \Gamma \\
& \mathcal{V}^{\prime}(\mathrm{B})=\{I I \mathrm{~B}, \mathrm{IVB}, \mathrm{VB}, \mathrm{VIII}\} \subset \sigma(\mathrm{B}) \Gamma \\
& \mathcal{V}^{\prime}(C)=\{I C, \| C, V I C, V I I C\} \subset \sigma(C) \Gamma \\
& \mathcal{V}^{\prime}(\mathrm{D})=\{\mathrm{IIID}, \mathrm{IVD}, \mathrm{VD}, \mathrm{VIIID}\} \subset \sigma(\mathrm{D}) \Gamma \\
& \text { and } \mathcal{V}^{\prime}=\mathcal{V}^{\prime}(A) \cup \mathcal{V}^{\prime}(B) \cup \mathcal{V}^{\prime}(C) \cup \mathcal{V}^{\prime}(D) \text {. }
\end{aligned}
$$

$$
\begin{aligned}
\mathcal{E}^{\prime}(\mathrm{A})=\{\mathrm{iiA}\} \subset \sigma(\mathrm{A}) \Gamma \\
\mathcal{E}^{\prime}(\mathrm{B})=\{\mathrm{iB}\} \subset \sigma(\mathrm{B}) \Gamma \\
\mathcal{E}^{\prime}(\mathrm{C})=\{\mathrm{iiC}\} \subset \sigma(\mathrm{C}) \Gamma \\
\mathcal{E}^{\prime}(\mathrm{D})=\{\mathrm{iD}\} \subset \sigma(\mathrm{C}) \Gamma \text { and } \mathcal{E}^{\prime}=\mathcal{E}^{\prime}(\mathrm{A}) \cup \mathcal{E}^{\prime}(\mathrm{B}) \cup \mathcal{E}^{\prime}(\mathrm{C}) \cup \mathcal{E}^{\prime}(\mathrm{D}) .
\end{aligned}
$$

Since $\kappa=1 \Gamma \mathcal{R}=\mathcal{S}$ (Section 2.2.1) $\Gamma$ and the elements of $\mathcal{E}^{\prime \prime}$ and $\mathcal{V}^{\prime \prime}$ are just those of $\mathcal{E}^{\prime}$ and $\mathcal{V}^{\prime}$. However $\Gamma$ the elements of $\mathcal{V}^{\prime \prime}$ are identified with their hulls (Section 2.2.2) Гshown in figure 28. Recall the hulls are marked with dark edges and light edges. The dark edges are black; those of relative level one are thick; those of relative level zero are thin. The light edges are gray.

At this point we orient the one-facets of our edges with arrows; to collapse the notation $\Gamma$ "side" our arrows: for example $\Gamma$ the hook on the head of each arrow on a pair of edges $+e \Gamma-e$ points to $-e$; we will consequently compound $\pm e$ for each $e \in \mathcal{E}^{\prime \prime}$. (These arrows first appear in figure 27 since $\mathcal{E}^{\prime}=\mathcal{E}^{\prime \prime}$ ).

\footnotetext{
${ }^{10}$ Again, this is unexceptional- most well known examples have $\kappa=1$
} 

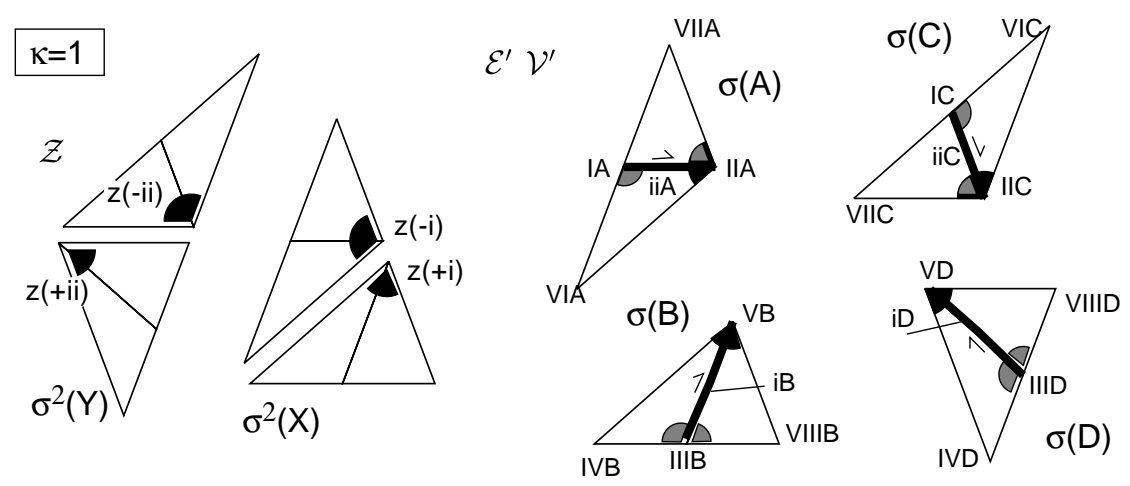

Figure 27: $\mathcal{Z} \Gamma \mathcal{V}^{\prime} \Gamma$ and $\mathcal{E}^{\prime}$

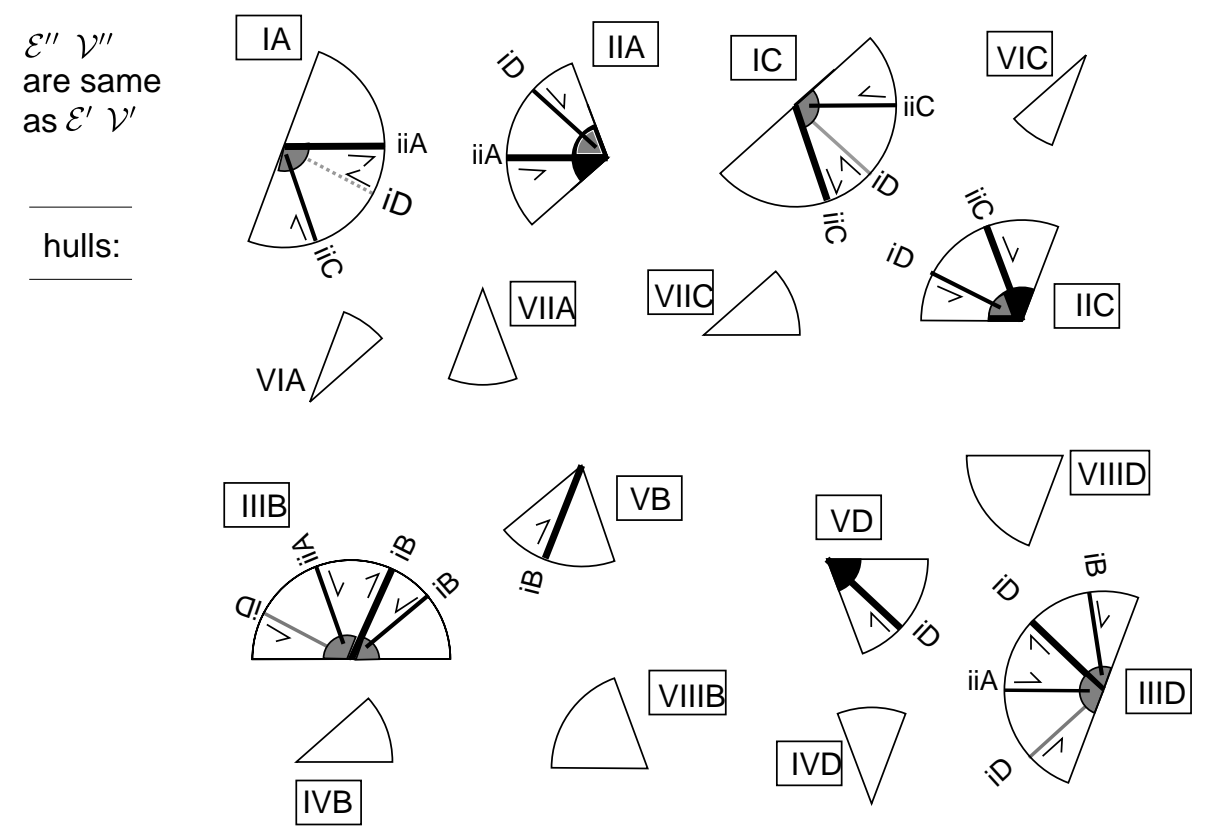

Figure 28: Vertex hulls for elements of $\mathcal{V}^{\prime \prime}$ 
We next define vertex wires for the mesovertices $2 \mathrm{~A}$ Г6ВГ1C and 4D (figure 29 and Section 2.3). Thus

$$
\begin{aligned}
& \mathrm{W}(2 \mathrm{~A})=\left[\mathrm{A}_{2 \mathrm{~A}} \bullet \mathrm{C}_{2 \mathrm{~A}}\right] \Gamma \\
& \mathrm{W}(6 \mathrm{~B})=\left[\mathrm{B}_{6 \mathrm{~B}} \bullet \mathrm{A}_{6 \mathrm{~B}}, \mathrm{D}_{6 \mathrm{~B}}\right] \Gamma \\
& \mathrm{W}(1 \mathrm{C})=\left[\mathrm{C}_{1 \mathrm{C}} \bullet \mathrm{D}_{1 \mathrm{C}}, \mathrm{A}_{1 \mathrm{C}}\right] \Gamma \\
& \mathrm{W}(4 \mathrm{D})=\left[\mathrm{D}_{4 \mathrm{D}} \bullet \mathrm{B}_{5 \mathrm{~B}}\right] .
\end{aligned}
$$

Recall that the elements of $\mathrm{W}(v)$ for any mesovertex $v$ are elements of $\mathcal{S} \Gamma$ but with additional information- the orientation of the tile and the mesovertex served.

$$
\begin{aligned}
& l(2 \mathrm{~A})=l(6 \mathrm{~B})=l(1 \mathrm{C})=l(4 \mathrm{D})=0 \\
& m(2 \mathrm{~A})=m(4 \mathrm{D})=1 \text { Гand } m(6 \mathrm{~B})=m(1 \mathrm{C})=2 .
\end{aligned}
$$

Recall the definition of $v_{n}$ given mesovertex $v$. Thus

$2 \mathrm{~A}_{0}=2 \mathrm{~A}$ and $2 \mathrm{~A}_{1}=2 \mathrm{C}_{2 \mathrm{~A}} \Gamma$

$6 \mathrm{~B}_{0}=6 \mathrm{~B} \Gamma 6 \mathrm{~B}_{1}=1 \mathrm{~A}_{6 \mathrm{~B}}$ and $6 \mathrm{~B}_{2}=6 \mathrm{D}_{6 \mathrm{~B}} \Gamma$

$1 C_{0}=1 C \Gamma 1 C_{1}=6 D_{1 C}$ and $1 C_{2}=1 A_{1 C}$ Tand

$4 \mathrm{D}_{0}=4 \mathrm{D}$ and $4 \mathrm{D}_{1}=5 \mathrm{~B}_{4 \mathrm{D}}$.

Thus 5 for epivertices $1 \mathrm{~A} \Gamma 5 \mathrm{~B} \Gamma 6 \mathrm{D}$ and $2 \mathrm{C}$ we define $\mathcal{U}$

$\mathcal{U}(1 \mathrm{~A})=\left\{\left(\mathcal{W}(6 \mathrm{~B})_{1}=\mathrm{A}_{6 \mathrm{~B}}, B\right),\left(\mathcal{W}(1 \mathrm{C})_{2}=\mathrm{A}_{1 \mathrm{C}}, \mathrm{C}\right)\right\} \Gamma$

$\left.\mathcal{U}(6 \mathrm{D})=\left\{\left(\mathcal{W}(6 \mathrm{~B})_{2}=\mathrm{D}_{6 \mathrm{~B}}, B\right),(\mathcal{W} 1 \mathrm{C})_{1}=\mathrm{D}_{1 \mathrm{C}}, \mathrm{C}\right)\right\} \Gamma$

$\mathcal{U}(2 \mathrm{C})=\left\{\left(\mathcal{W}(2 \mathrm{~A})_{1}=\mathrm{C}_{2 \mathrm{~A}}, A\right)\right\} \Gamma$ and

$\mathcal{U}(5 \mathrm{~B})=\left\{\left(\mathcal{W}(4 \mathrm{D})_{1}=\mathrm{B}_{4 \mathrm{D}}, D\right)\right\}$.

We next turn to the packets any given supertile may have to carry (Section 2.4). Since our wires are short $\Gamma$ our epivertices few $\Gamma$ and $k=1 \Gamma$ these are blessedly few. We'll collapse the notation as we goTreusing our labels in $\mathcal{W}$ as names of supertile packets.

$$
\begin{aligned}
& \mathcal{Q}(\mathrm{A})=\left\{\left[\mathrm{A},\left(\mathcal{W}(6 \mathrm{~B})_{1}=\mathrm{A}_{6 \mathrm{~B}}, B\right)\right]=\mathrm{A}_{6 \mathrm{~B}},\left[\mathrm{~A},\left(\mathcal{W}(1 \mathrm{C})_{2}=\mathrm{A}_{1 \mathrm{C}}, \mathrm{C}\right)\right]=\mathrm{A}_{1 \mathrm{C}}\right\} \Gamma \\
& \mathcal{Q}(\mathrm{B})=\left\{\left[\mathrm{B},\left(\mathcal{W}(4 \mathrm{D})_{1}=\mathrm{B}_{4 \mathrm{D}}, D\right)\right]=\mathrm{B}_{4 \mathrm{D}}\right\} \Gamma \\
& \mathcal{Q}(\mathrm{C})=\left\{\left[\mathrm{C},\left(\mathcal{W}(2 \mathrm{~A})_{1}=\mathrm{C}_{2 \mathrm{~A}}, A\right)\right]=\mathrm{C}_{2 \mathrm{~A}}\right\} \Gamma \\
& \mathcal{Q}(\mathrm{D})=\left\{\left[\mathrm{D},\left(\mathcal{W}(6 \mathrm{~B})_{2}=\mathrm{D}_{6 \mathrm{~B}}, B\right)\right]=\mathrm{D}_{6 \mathrm{~B}},\left[\mathrm{D},\left(\mathcal{W}(1 \mathrm{C})_{1}=\mathrm{D}_{1 \mathrm{C}}, C\right)\right]=\mathrm{D}_{1 \mathrm{C}}\right\}
\end{aligned}
$$

Next $\Gamma$ we give the edge packets. Again $\Gamma$ one is relieved that the example is simple. We provide reasonable names for the packets as we go. Possible edge packets are:

$$
\begin{aligned}
& \mathcal{P}\left(\mathcal{E}^{\prime \prime}\right)=\left\{\left[i i \mathrm{~A}, \mathrm{~A}_{1} \mathrm{C}\right]=\mathrm{ii}_{1 \mathrm{C}} \Gamma\right. \\
& {\left[\mathrm{ii}, \mathrm{A}_{6 \mathrm{~B}}\right]=\mathrm{ii} \mathrm{A}_{6 \mathrm{~B}} \Gamma} \\
& {\left[\mathrm{iB}, \mathrm{B}_{4 \mathrm{D}}\right]=\mathrm{iB}_{4 \mathrm{D}}} \\
& {\left[\mathrm{iiC}, \mathrm{C}_{2 \mathrm{~A}}\right]=\mathrm{ii}_{2 \mathrm{~A}} \Gamma} \\
& \left.\left[\mathrm{iD}, \mathrm{D}_{6 \mathrm{~B}}\right]=\mathrm{iD}_{6 \mathrm{~B}},\left[\mathrm{iD}, \mathrm{D}_{1 \mathrm{C}}\right]=\mathrm{iD}_{1 \mathrm{C}}\right\}
\end{aligned}
$$



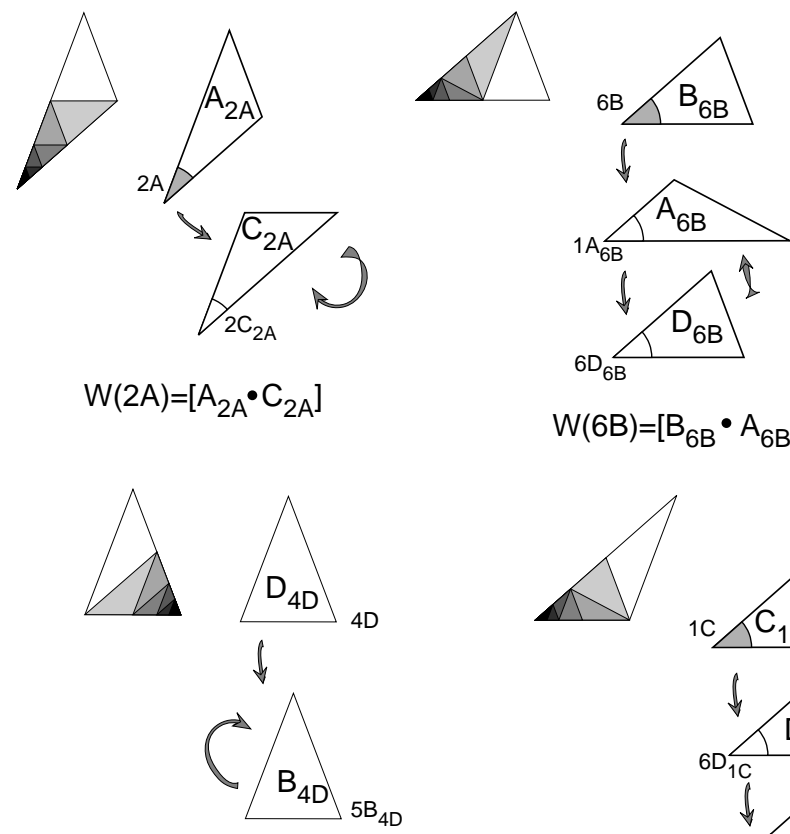

$$
W(6 B)=\left[B_{6 B} \cdot A_{6 B}, D_{6 B}\right]
$$

$W(4 D)=\left[D_{4 D^{\bullet}} B_{4 D}\right]$

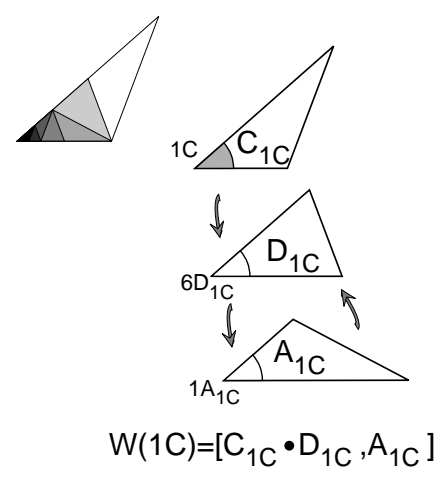

Figure 29: Vertex wires for the mesovertices 
Similarly $\Gamma$ we define vertex packets. Recall we do not bother defining these for vertices unless they meet the skeleton of the tile in $\mathcal{S}$ to which they belong. Thus $\Gamma$ we do not define packets for VIAГ VIIAIVIIIBГ IVВГ VIC Г VIIC Г VIIID IVD.

The vertex packets are:

$$
\begin{aligned}
& \mathcal{P}\left(\mathcal{V}^{\prime \prime}\right)=\left\{\left[I A, A_{1 C}\right]=I A_{1 C},\left[I I A, A_{1 C}\right]=I I A_{1 C} \Gamma\right. \\
& {\left[I A, A_{6 B}\right]=I A_{6 B},\left[I I A, A_{6 B}\right]=\| A_{6 B},} \\
& {\left[I I I B, B_{4 D}\right]=I I I B_{4 D},\left[V B, B_{4 D}\right]=V B_{4 D}} \\
& {\left[I C, C_{2 A}\right]=I C_{2 A},\left[I I C, C_{2 A}\right]=I I C C_{2 A}} \\
& {\left[I I I D, D_{6 B}\right]=I I D_{6 B},\left[V D, D_{6 B}\right]=V_{6 B}} \\
& \left.\left[I I I D, D_{1 C}\right]=I I D_{1 C},\left[V D, D_{1 C}\right]=V D_{1 C}\right\}
\end{aligned}
$$

We turn to the tiles. First $\Gamma$ in figure $30 \Gamma$ the unmarked tiles themselves are shown (Section 3). The vertex tiles will be made of the various sectors illustrated; they will be half disks when fully assembled (because no element of $V$ lies in the interior of a $\sigma(\mathrm{X})$ or $\sigma(\mathrm{Y})$ none of our vertex tiles will be full disks). Following this $\Gamma$ the small tiles $\Gamma$ marked with supertile packets and indications of the interior edges Tare shown (figure 31 ).
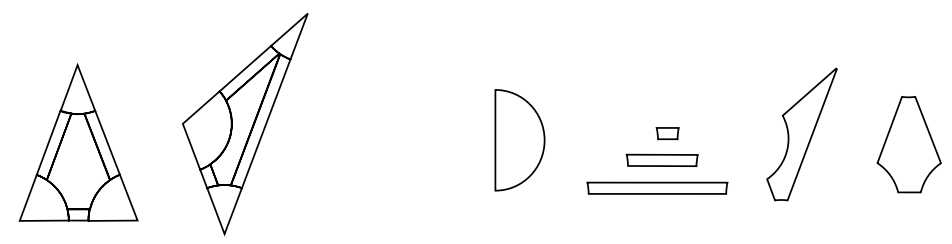

Figure 30: The unmarked tiles
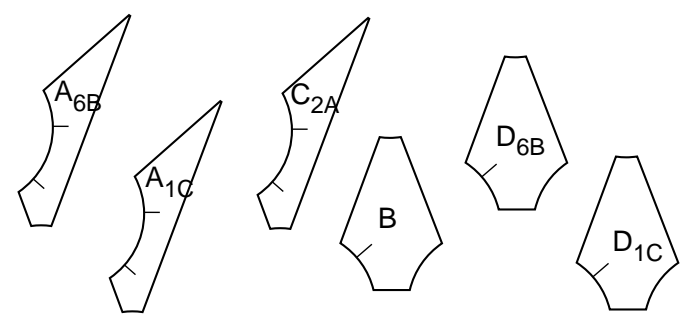

Figure 31: The small tiles marked with supertile packets

In the next illustration $\Gamma$ the edge tiles are marked (figure 32 ). Note that 
we allow orientation reversing isometries of our edge-tiles. If we regard the edge packets and orientations as residing on little tiles we implant into our edge tiles $\Gamma$ we can reduce the number of tiles needed here from $9 \times 2 \times 3=54$ to $9+1+3=13$.

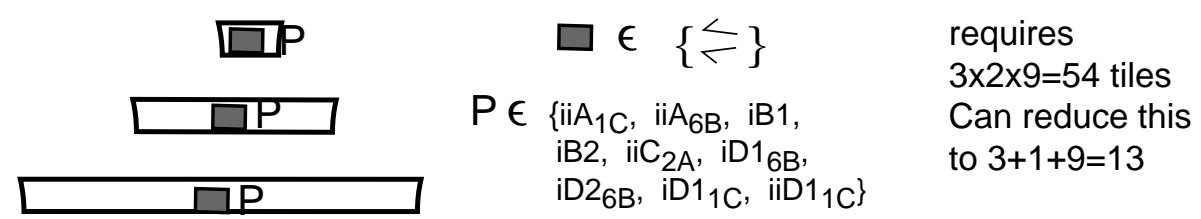

Figure 32: Marked edge tiles

The vertex hulls are next assembled into actual vertex tiles. In this example $\Gamma$ there are not very many possibilities allowed by the compatibility rules (Section 3.3). In particular $\Gamma$ there are only six basic kinds of stack allowed; each can be capped off by any of the $9 \times 4$ overpass decorations (each corresponding to an edge packet) giving two hundred sixteen vertex tiles. We can reduce this to $6+9+1=16$ tiles by breaking the tiles up into independent pieces as shown in figure 33 .

Note that we thus have a grand total of six small tiles $\Gamma$ fifty-four edge tiles $\Gamma$ and two hundred sixteen vertex tiles $\Gamma$ for a total of 276 tiles altogether $\Gamma$ or if we break the tiles up furtherГsix small tiles $\Gamma$ three blank edge-tiles $\Gamma$ nine edgepacket tiles $\Gamma$ one tiles indicating orientation of the edges $\Gamma$ and six vertex tiles $\Gamma$ for a total of twenty five tiles. With imagination we might reduce this number even further. 

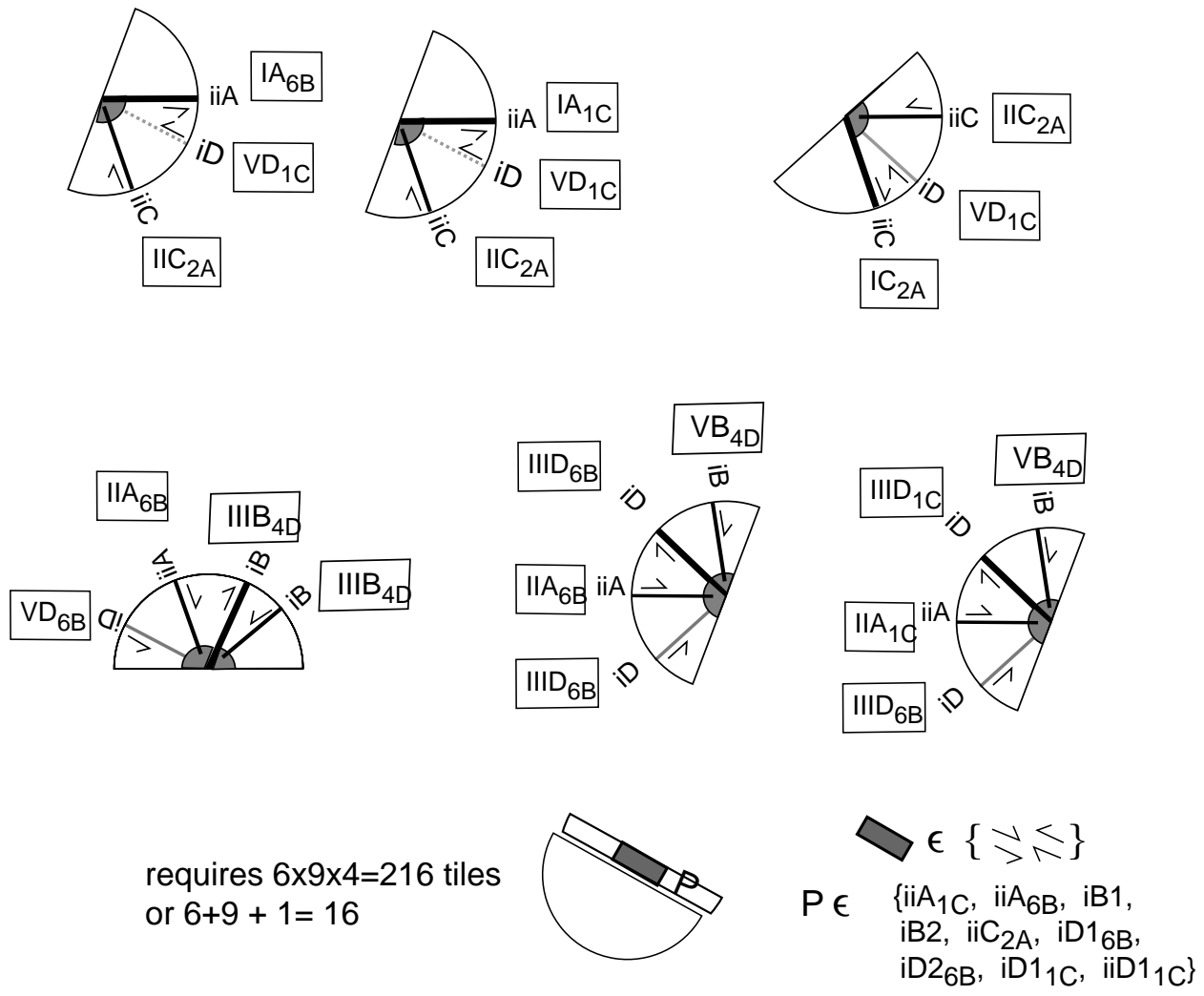

Figure 33: Marked vertex tilesToverpasses 


\section{Formalisms}

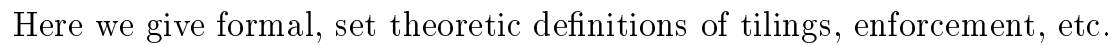

\section{C.1 Prototiles $\mathcal{T}$}

Let $\mathrm{N}$ be the natural numbers $\Gamma\{1,2 \ldots\}$. We take for our space and congruences $\Gamma$ $\mathrm{n}$-dimensional Euclidean space $\mathrm{E}^{\mathrm{n}} \Gamma 1<\mathrm{n} \in \mathrm{N}$. $\mathcal{G}$ will be the set of Euclidean isometries on $E^{n}$.

A prototile $\mathrm{A}$ is a $\mathrm{n}$-dimensional compact set $\Lambda \in \mathrm{E}^{\mathrm{n}} \Gamma$ endowed with a map $\mu_{\mathrm{A}}: \Lambda \rightarrow \mathcal{X}$ to some set $\mathcal{X}$ of combinatorial information ${ }^{11} \Lambda$ will be called the underlying set of $\mathrm{A}$. Generally $\Gamma \mathcal{X}$ will be built from labels $\Gamma$ as in Sections $2 \Gamma 3$ and 3.5. In Section 1.2 we place further restrictions on $\Lambda$ if the prototiles are for a "substitution tiling".

A tile is a congruent image $\mathrm{BA} \Gamma \mathrm{B} \in \mathcal{G}$ of a prototile $\mathrm{A}$. We extend $\mu$ to act on tiles: $\mu_{\mathrm{BA}}(x)=\mu_{\mathrm{A}}\left(\mathrm{B}^{-1}(x)\right)$.

A tiling $\cup \mathrm{B}_{i} \mathrm{~A}_{i}$ of $X \subset \mathrm{E}^{\mathrm{n}}$ by prototiles $\mathcal{T}$ satisfies:

$X=\cup \mathrm{B}_{i} \mathrm{~A}_{i} \Gamma$

for $i \neq j \Gamma \mathrm{B}_{i} \mathrm{~A}_{i}$ and $\mathrm{B}_{j} \mathrm{~A}_{j}$ have disjoint interiors $\Gamma$

and if $x \in \mathrm{B}_{i} \mathrm{~A}_{i} \cap \mathrm{B}_{j} \mathrm{~A}_{j} \Gamma$ then $\mu_{\mathrm{B}_{i} \mathrm{~A}_{i}}(x)=\mu_{\mathrm{B}_{j} \mathrm{~A}_{j}}(x)$.

If we do not specify $X \Gamma$ we assume $X=E^{n}$. The last condition assures that $\mu$ can be defined on a tiling: $\mu_{\cup \mathrm{B}_{i} \mathrm{~A}_{i}}(x)=\mu_{\mathrm{B}_{j} \mathrm{~A}_{j}}(x)$ for any $\mathrm{B}_{j} \mathrm{~A}_{j}$ containing $x$. The last condition is in fact a "matching rule" $\Gamma$ but is weaker than we would like in general. ${ }^{12}$ Note that $\mu$ is defined on any congruence $C \in \mathcal{G}$ of a tiling: $\mu \mathrm{CUB}_{i} \mathrm{~A}_{i}(x)=\mu_{\cup \mathrm{B}_{i} \mathrm{~A}_{i}}\left(\mathrm{C}^{-1} x\right)=\mu_{\cup \mathrm{CB}_{i} \mathrm{~A}_{i}}(x)$.

A set of tilings of $X$ by prototiles $\mathcal{T}$ Tperhaps with further restrictions $\Gamma$ is a species of tilings. ${ }^{13}$

\footnotetext{
${ }^{11}$ such as color (as in figure 2), the position of each point in a tile, the specific facet of $\Lambda$ to which each point belongs, etc. We place no restrictions on $\mathcal{X}$ and $\mu$ could have a very rich or very simple structure.

${ }^{12}$ Actually, the definitions so far are sufficient to define rules that involve matching "notches", as with a jig-saw puzzle, or matching colors on edges, as in Wang's papers. Moreover, such rules are sufficient for our construction. However, the condition is too weak to define local matching rules in much generality: in particular, some rules may involve complicated matchings across edges in tilings that are not "edge-to-edge", or may require a local atlas. The definition of $\mu$ also allows us to precisely define when a "matching rule tiling enforces a substitution tiling".

${ }^{13}$ We primarily use $\mu$ to define equivalence between species of tilings. Roughly speaking, two tilings are to be equivalent if they give the same map $\mu$ from the points of the tilings to some combinatorial space $\mathcal{X}$.
} 
"Configurations" are often defined as a tiling of some compact $X \Gamma$ but for later convenience we take: A configuration is a subset $D \subset \cup B_{i} A_{i}$ of points in a tiling; thus $\mu$ is also defined on points in a configuration: $\mu_{\mathrm{D}}=\left.\mu_{\cup B_{i} A_{i}}\right|_{D}$. For any configurations $C, D \Gamma$ the symbol " $\subset$ " in $\mathrm{C} \subset \mathrm{D}$ connotes not only that for all $x \in \mathrm{C} \Gamma x \in \mathrm{D} \Gamma$ but also that $\mu_{\mathrm{C}}(x)=\mu_{\mathrm{D}}(x)$. Note $\Gamma$ of course $\Gamma$ that tilings are configurations.

A matching rule for a tiling of $X$ with prototiles $\mathcal{T}$ is simply a bounded configuration.

A tiling $\cup B_{i} A_{i}$ satisfies a set $\mathcal{M}$ of matching rules if and only if there exist congruences of the matching rules $\left\{C_{j} D_{j}\right\} \Gamma\left\{C_{j}\right\} \subset \mathcal{G}$ and $\left\{D_{j}\right\} \subset \mathcal{M} \Gamma$ such that $\cup \mathrm{B}_{i} \mathrm{~A}_{i} \subset \cup \mathrm{C}_{j} \mathrm{D}_{j}$ and $\mu \cup \mathrm{B}_{i} \mathrm{~A}_{i}(x)=\mu_{\mathrm{C}_{j} \mathrm{D}_{j}}(x)$ for all $x \in \cup \mathrm{B}_{i} \mathrm{~A}_{i} \Gamma$ for all $C_{j} \mathrm{D}_{j}$ containing $x$.

A matching rule tiling $\left(\mathcal{M} \Gamma \mathcal{T}^{\prime} \Gamma \mathcal{X} \Gamma \mu\right) \Gamma$ or $\mathcal{M}$-tiling $\Gamma$ is the species of tilings of $\mathrm{E}^{\mathrm{n}} \Gamma$ with prototiles $\mathcal{T}^{\prime}$ with $\mu_{\mathrm{A}}: \mathrm{A} \rightarrow \mathcal{X}$ for each $\mathrm{A} \in \mathcal{T}^{\prime} \Gamma$ that satisfy matching rules $\mathcal{M}$.

For example $\Gamma$ in figure $2 \Gamma$ we have two tiles in $T^{\prime} \Gamma \mathcal{X}=\{$ Black, Gray, White, Border \}; $\mu$ takes points marked "black" in the figure to BlackГetc.; points on the boundary between colors are sent to Border and points on the thick lines are sent to Bold. As the price of formality we can take small neighborhoods as shown $\Gamma$ plus the interiors of our tiles $\Gamma$ plus small neighborhoods of the vertices in a tiling.

\section{C.2 Enforcement and labelings}

We now define "enforcement" of a substitution tiling by matching rules. The following approach is meant to include any reasonable definition of enforcement and no others. In particular $\Gamma$ we have tried to avoid ambiguity in any of our terms.

Let $(\mathcal{T} \Gamma \sigma \mathrm{S})$ be a substitution tiling and $\mathcal{X}$ some arbitrary set $\Gamma$ with a projection $\pi: \mathcal{X} \rightarrow \mathcal{T} \cup\{\partial\}$. Recall that the $\mu_{\mathrm{A}} \Gamma \mathrm{A} \in \mathcal{T}$ have not been explicitly chosen $\Gamma$ but conditions have been made on these $\mu_{\mathrm{A}}$.

A labeling of the substitution tiling $(\mathcal{T} \Gamma \sigma \Gamma \mathcal{S})$ is a set $\mathcal{X}$ as above and a set of maps $\lambda_{\cup \mathrm{B}_{i} \mathrm{~A}_{i}}: \cup \mathrm{B}_{i} \mathrm{~A}_{i} \rightarrow \mathcal{X} \Gamma$ defined for all tilings $\cup \mathrm{B}_{i} \mathrm{~A}_{i} \in \Gamma \Gamma$ such that: 
i) $\pi\left(\lambda_{\cup \mathrm{B}_{i} \mathrm{~A}_{i}}(x)\right)=\mu \cup \mathrm{B}_{i} \mathrm{~A}_{i}(x)$ for all $x \in \cup \mathrm{B}_{i} \mathrm{~A}_{i}$ Tfor all tilings $\cup \mathrm{B}_{i} \mathrm{~A}_{i} \in \Gamma$;

ii) for each supertile $\sigma^{n}(\mathrm{~A}) \Gamma$ let $\lambda\left(\sigma^{n}(\mathrm{~A})\right)=\left\{\left.\lambda_{\cup \mathrm{B}_{i} \mathrm{~A}_{i}}\right|_{\mathrm{B} \sigma^{n}(\mathrm{~A})} \mathrm{B}: \sigma^{n}(\mathrm{~A}) \rightarrow \mathcal{X} \mid \sigma^{n}(\mathrm{~A}) \subset\right.$ $\left.\cup \mathrm{B}_{i} \mathrm{~A}_{i} \in \Gamma\right\}$; then we require $\left|\lambda\left(\sigma^{n}(\mathrm{~A})\right)\right| \in \mathrm{N}$.

Once we have a labeling for $(\mathcal{T} \Gamma \sigma \mathbb{S}) \Gamma$ we define a well-formed supertile of level $n$ in $\left(\mathcal{M} \Gamma \mathcal{T}^{\prime} \Gamma \mathcal{X} \Gamma \mu^{\prime}\right)$ to be a configuration $X$ of tiles in $\mathcal{T}^{\prime} \Gamma$ satisfying $\mathcal{M} \Gamma$ such that there exist $\mathrm{A} \in \mathcal{T} \Gamma \mathrm{B} \in \mathcal{G} \Gamma$ and $\lambda^{\prime} \in \lambda\left(\sigma^{n}(\mathrm{~A})\right)$ with

$\mathrm{B} X=\sigma^{n}(\mathrm{~A})$ and for all $x \in \mathrm{X} \Gamma$

$\lambda_{\sigma^{n}(\mathrm{~A})}^{\prime}(\mathrm{B} x)=\mu_{X}^{\prime}(x)$.

A matching rule tiling $\left(\mathcal{M} \Gamma \mathcal{T}^{\prime} \Gamma \mathcal{X} \Gamma \mu^{\prime}\right)$ enforces a substitution tiling $(\mathcal{T} \Gamma \sigma \Gamma \mathcal{S})$ if and only if one can define a labeling on $(\mathcal{T} \Gamma \sigma \Gamma \mathcal{S})$ such that for every $n \in \mathrm{N} \Gamma$ every point in the interior of any tile in any tiling in $\left(\mathcal{M} \Gamma \mathcal{T}^{\prime} \Gamma \mathcal{X} \Gamma \mu\right)$ lies in a unique well-formed supertile of level $n$.

A substitution tiling $(\mathcal{T} \Gamma \sigma \mathcal{S})$ is enforced by matching rules if and only if there is a finite set of matching rules $\mathcal{M} \Gamma$ and a finite set $\mathcal{T}^{\prime}$ of prototiles endowed with maps $\mu_{A}^{\prime}$ with images in some $\mathcal{X}$ such that $\left(\mathcal{M} \Gamma \mathcal{T}^{\prime} \Gamma \mathcal{X} \Gamma \mu^{\prime}\right)$ enforces $(\mathcal{T} \Gamma \sigma \Gamma$ $\mathcal{S})$.

\section{C.3 Equivalence}

We point out that our definition of enforcement is substantially the same as those used elsewhere- particularly by Mozes [Mos] and Radin [Rad].

The following is adapted from [Mos]. To make sense of this every tiling has some vertex at the origin of $\mathrm{E}^{\mathrm{n}}$; thus there are only countably many translations of a tiling.

Lemma C.1 In any $(\mathcal{T}, \sigma, \mathcal{S})$ with unique decomposition, under any $\mathcal{G}$-invariant probability measure on $(\mathcal{T}, \sigma, \mathcal{S})$, the set of tilings with connected hierarchy has measure 1.

Proof For any set $S \subset \mathrm{E}^{\mathrm{n}} \Gamma$ let $T(S)$ be the set of tilings for which $S$ is the boundary between infinite level supertiles. If $T(S)$ is not emptyГ $S$ must include a $k$-plane $\Gamma \leq k<\mathrm{n} \Gamma$ from which radiate $(\mathrm{n}-1)$-dimensional sectors (this follows from examining the boundary of an infinite level supertile). The $T(S)$ are disjoint and their union is the set of all tilings in $(\mathcal{T}\lceil\Gamma \mathcal{S})$ with disconnected hierarchy. Let $m$ be any $\mathcal{G}$-invariant probability measure on $(\mathcal{T}\lceil\sigma \mathcal{S})$. For any 
$S \Gamma$ there is a countable collection of translations $\left\{\mathrm{B}_{i}\right\} \subset \mathcal{G}$ none of which leaves $S$ invariant. So $T\left(\mathrm{~B}_{i} S\right)$ is disjoint from $T\left(\mathrm{~B}_{j} S\right)$ for all $i \neq j$; but $m\left(T\left(\mathrm{~B}_{i} S\right)\right)=$ $m\left(T\left(\mathrm{~B}_{j} S\right)\right)$ for all $i, j \in \mathrm{N}$. And since the total measure is $1 \Gamma$ and the measure of each $T\left(\mathrm{~B}_{i} S\right)$ must be zero. This is regardless of the choice of $S \Gamma$ and so the set of tilings with connected hierarchy has measure 1 .

qed

Lemma C.2 Any tiling in $(\mathcal{T}, \sigma, \mathcal{S})$ that has unique and connected hierarchy is in unique correspondence with a tiling in $\left(\mathcal{M}, \mathcal{T}^{\prime}\right)$.

That is $\Gamma$ if the hierarchy is unique $\Gamma$ and there are no infinite fault lines $\Gamma$ only one tiling satisfying the matching rules will correspond to the tiling in $(\mathcal{T} \Gamma \sigma \Gamma$ $\mathcal{S})$.

Proof In such a tiling every tile lies in a unique supertile of level $n \Gamma$ for all $n \in \mathrm{N}$; moreover every pair of tiles lies in some supertile of some level. In particular $\Gamma$ any matching rule tiling corresponding to our tiling in $(T \Gamma \sigma \Gamma \mathcal{S})$ must have every edge label fixed $\Gamma$ since the edge must lie in the interior of some supertile and thus must lie on some skeleton.

qed 


\section{References}

[Ber] R. BergerГThe undecidability of the domino problemГMemoirs Am. Math. Soc. 66 (1966).

[CL] J.H. Conway and J.C. LagariusTTiling with polyominoes and combinatorial group theoryГJ. of Comb. Theory Ser. A 53 (1990)Г183-208.

[dBr] N.G. de BruijnГ Algebraic theory of Penrose's non-periodic tilings $\Gamma$ Nederl. Akad. Wentensch Proc. Ser. A 84 (1981)Г39-66.

[Dan] L. Danzer $A$ family of 3D-spacefillers not permitting any periodic or quasiperiodic tiling preprint.

[Dan2] L. DanzerГpersonal communication.

[Eps] Epstein et al.ГWord processing in groupsГJones and Bartlett (1992).

[Godr] C. GodrecheГThe sphinx: a limit periodic tiling of the planeГJ. Phys. A: Math. Gen. 221989ГL1163-L1166.

[GS] C. Goodman-Strauss $\Gamma A n$ aperiodic tiling of $\mathrm{E}^{\mathrm{n}}$ for all $\mathrm{n}>1$ Tpreprint.

[GrSh] B. Grunbaum and G.C. Shepherd $\Gamma$ Tilings and patterns $\Gamma$ W.H. Freeman and Co. (1989).

[Ken] R. KenyonГSelf similar tilings $\Gamma$ thesis $\Gamma$ Princeton UniversityГ1990.

[Le] T.T.Q. LeTLocal rules for quasiperiodic tilings $\Gamma$ preprint.

[Luc] J.M. LuckTAperiodic structures: geometry, diffraction spectra, and physical properties to appear in Fund. Prob. in Stat. Mech VIII.

[Moz] S. MozesГTilings, substitution systems and dynamical systems generated by themГJ. D'Analyse Math. 53 (1989)Г139-186.

[Pen] R. Penrose $\Gamma$ The role of aesthetics in pure and applied mathematical research $\Gamma$ Bull. Inst. of Math. and its Appl. 10 (1974) 266-71.

[Rad] C. Radin $\Gamma$ The pinwheel tilings of the planeГAnnals of Math. 139 (1994)Г 661-702.

[Rad2] C. RadinTpersonal communication.

[RobE] E.A. RobinsonTpersonal communication. 
[Rob] R. RobinsonTUndecidability and nonperiodicity of tilings in the planeГInv. Math. 12 (1971)Г177.

[Sad] L. SadunTSome generalizations of the pinwheel tiling $\Gamma$ preprint.

[Sen] M. SenechalГ Quasicrystals and geometryГ Cambridge University Press (1995).

[SO] P. Steinhardt and S. Ostlund $\Gamma$ The physics of quasicrystals $\Gamma$ World Scientific (1987).

[Soc] J. SocolarTpersonal communication.

[Sol] B. Solomyak $\Gamma$ Non-periodic self-similar tilings have unique decomposition $\Gamma$ preprint.

[Thu] W. ThurstonTGroups, tilings and finite state automata: Summer 1989 AMS colloquim lectures $\Gamma \mathrm{GCG} 1 \Gamma \mathrm{Teometry}$ Center.

[Wal] P. Walters Teditor $\Gamma$ Symbolic dynamics and its applications $\Gamma$ Contemporary Mathematics 135 (1992). 


\section{Contents}

0.1 A sketch of the techniques . . . . . . . . . . 4

1 Definitions $\quad 11$

1.1 Tiles, matching rules, matching rule tilings . . . . . . . 11

1.2 A similarity $\sigma$ and substitutions $\mathcal{S} \ldots \ldots \ldots \ldots \ldots$

1.3 Enforcement . . . . . . . . . . . . . . . . . . 17

1.4 Addresses . . . . . . . . . . . . . . . . . . . . . 18

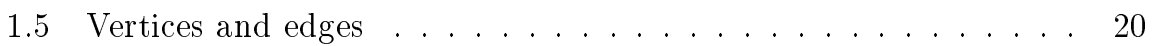

1.6 Epi-TMeso- and Endo- vertices . . . . . . . . . . . . 22

2 Selecting structures in a substitution tiling 24

2.1 Selecting structures to form skeletons . . . . . . . . . . . . 24

2.1.1 Selecting vertices $\mathcal{V}$ and edges $\mathcal{E} \ldots \ldots \ldots \ldots$

2.1 .2 Selecting sites $\mathcal{Z} \ldots \ldots \ldots \ldots \ldots \ldots$

2.1 .3 Selecting $\kappa \ldots \ldots \ldots \ldots \ldots$

2.1.4 Selecting vertices $\mathcal{V}^{\prime}$ and edges $\mathcal{E}^{\prime}$ of skeletons . . . . . 27

2.2 Keys . . . . . . . . . . . . . . . . 28

2.2 .1 Supertile keys $\mathcal{R} \ldots \ldots \ldots \ldots \ldots$

2.2 .2 Edge keys $\mathcal{E}^{\prime \prime} \ldots \ldots \ldots \ldots \ldots \ldots$

2.2 .3 Vertex hulls $\mathcal{V}^{\prime \prime} \ldots \ldots \ldots \ldots \ldots \ldots \ldots$

2.3 Vertex-wires $\mathcal{W}$ and wire-keys $\mathcal{U} \ldots \ldots \ldots \ldots \ldots$

2.4 Supertile packets $\mathcal{Q}$ Tedge- and vertex-packets $\mathcal{P} \ldots \ldots \ldots . .35$

3 Creating tiles and markings 39

3.1 Creating new unmarked tiles . . . . . . . . . . . . 39

3.2 Vertex tiles and vertex markings . . . . . . . . . . . . 41

3.3 Compatibility rules . . . . . . . . . . . . . . . 43

3.4 Small tiles . . . . . . . . . . . . . . . . . . . . 44

3.5 Matching Rules $\mathcal{M}$ and tiles $\mathcal{T}^{\prime} \ldots \ldots \ldots \ldots \ldots$. . . . . . . 44

3.6 The labeling $\lambda \ldots \ldots \ldots \ldots \ldots \ldots \ldots$

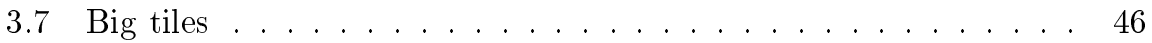

4 The proof of Theorem $1 \quad 47$

A Survey of substitution tilings $\quad 51$

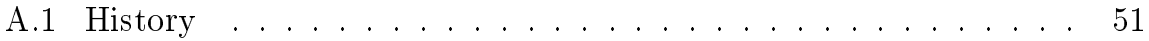

A.2 Well known examples . . . . . . . . . . . . . 54

A.3 Triangles . . . . . . . . . . . . . . . . . 54

A.4 Higher dimensional examples . . . . . . . . . . . . 57

A.5 Products of symbolic dynamical systems . . . . . . . . . 57

A.6 Non- vertex-to-vertex examples . . . . . . . . . . . . . . 58

A.7 Unique decomposition . . . . . . . . . . . . . . 58

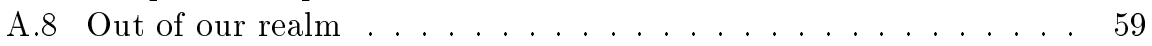


B A simple example $\quad 62$

$\begin{array}{ll}\text { C Formalisms } & \mathbf{7 0}\end{array}$

C.1 Prototiles $\mathcal{T} \ldots \ldots \ldots \ldots \ldots \ldots \ldots$. . . . . . . . . 70

C.2 Enforcement and labelings . . . . . . . . . . . . 71

C.3 Equivalence . . . . . . . . . . . . . . . . 72

Chaim Goodman-Strauss

Dept. of Mathematics

University of Arkansas

Fayetteville, AR 72701

cgstraus@comp.uark.edu

http://www.geom.umn.edu/ strauss 